\title{
Embodying Gender/sex Identity during Infancy: A Theory and Preliminary Findings
}

Anne Fausto-Sterling, Jihyun Sung, Malika Hale, Gopika Krishna, and Matthew Lin

Brown University, Sungkyunkwan University and New York University Langone Medical Center

Anne Fausto-Sterling, Malika Hale, Department of Molecular Biology, Cell Biology and Biochemistry, Brown University, Gopika Krishna, Alpert Medical School, Brown University, Matthew Lin, New York University Langone Medical Center, and Jihyun Sung, Department of Child Psychology and Education, Sungkyunkwan University

Correspondence should be addressed to:

Anne Fausto-Sterling and Jihyun Sung, Department of Child Psychology and Education, Sungkyunkwan University, 25-2 Sungkyunkwan-ro, Jongno-gu, Seoul, South Korea

E-mail: afs@brown.edu; sungih@skku.edu

Author's contributions:

Professor Fausto-Sterling was the PI and director of this project and drafted the original manuscript. Professor Sung, who is co-corresponding author, developed the coding system, supervised data collection, and contributed significantly to the final manuscript draft. Dr. Krishna did the work on assisted standing as a senior thesis at Brown University; Ms. Hale developed and performed the longitudinal analyses and produced the original graphs as a research assistant in Professor Fausto-Sterling's lab and Dr. Lin analyzed motor development as part of his senior thesis at Brown University. 


\section{Table of Contents}

\section{Abstract \\ 3 Introduction \\ 4 New Terminology}

$5 \quad$ Modeling Identity: Fixed Trait Versus Dynamic Process

6 Developmental Psychology and Gender/sex Formation

7 Studying Gender/sex as a Dynamic Process

9 Comparing Models and Assumptions

\section{Constructing a Timeline}

11 Neural Processes

11 Dyadic Behaviors and Presymbolic Gender/sex Skills

12 Statistics and Chunks: Presymbolic Input Becomes Embodied Via Touch and Movement

13 Symbolic Gender/sex

\section{Methods}

15 Participants, Taping and Initial Data Collection

15 Code Descriptions

17 Rater Reliability

18 Analyses and Graphing

19 Study Family Anonymity

\section{Results}

20 Large Gender/sex Differences

24 Talking and Touching while Assisting Locomotion

30 What are They Talking About? What are They Doing?

\section{Conclusion: wrapping things up}

31 Discussion of Reported Data

34 An Embodiment Model (To be a Girl or a Boy? That is the Question)

\section{Future Directions}

\section{Acknowledgements}

38 References

\section{Appendix 1}

44 Coding and Team Organization

44 Statistical Concerns

45 Average Kappa Scores

46 Coding Manual

53 References for Online Resource 1

\section{Appendix 2}




\section{Abstract}

In this paper we tried to loosen the following knot: at some point in mid toddlerhood, children evidence a gender/ sex-a personal sense of group belonging which also predicts individual behaviors and wants-play, dress and peer preferences. Yet at birth, although individual pattern differences in embodied responses to new stimuli exist, these do not appear to correlate with gender/sex (Kagan, 1994), and few measurable differences exist between female-designated and male-designated infants. As gender/ sex becomes visible and measurable, it already seems to emanate from deep within the body. How does individualapparently non-gender/sex-correlated-- variability turn into individual gender/sex identity? How do measurable gender/sex-related group differences appear? In this essay we contrast process-based theories of gender/sex identity and development with theories that posit identity as an inherent trait. From our own research, we offer data that demonstrate differences in behavior and infant handling in mother-son compared to mother-daughter dyads. We use these findings and others in the literature to develop a theory of embodiment and conclude with a proposal to refocus research in the field of infant gender/sex development. Specifically, we urge the use of longitudinal, multi-disciplinary research designs and analytical tools that emphasize emerging properties and developmental process.

Keywords: identity, gender/sex, infant development, dynamic systems, process theory, embodiment

\section{Introduction}

Identities related to gender and orientation have proliferated. Children and adolescents declare (sometimes in contradiction to their genitalia or gonadal anatomy) that they are a boy or a girl, or tell peers or parents that they identify as trans, gender-queer, bigender, agender, cis-gender, etc. How can we understand this rapidly transforming landscape? Professional organizations concerned with child development offer definitions of gender identity. For example, the authors of a pamphlet published by the American Psychological Association (APA) defined gender identity as "A person's deeply-felt, inherent sense of being a boy, a man, or male; a girl, a woman, or female; or an alternative gender (e.g., genderqueer, gender nonconforming, gender neutral) that may or may not correspond to a person's sex assigned at birth or to a person's primary or secondary sex characteristics. Since gender identity is internal, a person's gender identity is not necessarily visible to others" (Schlittler, 2017, p. 4 of 7). Similarly, a pediatrician-generated website offered that "When children are able to express themselves, they will declare themselves to be a boy or a girl (or sometimes something in between); this is their "gender identity" (Healthychildren.Org, 2015)". Recently, a group of tenured academics published an on-line guide for discussing "transgender people, experiences, identities, backgrounds and existences in academic talks" (van Anders, Galupo, Irwin, Twist, \& Reynolds, 2019, p. 1). In the document they wrote "It is important to recognize that trans experiences, identities, backgrounds, and existences are heterogeneous in whether trans is included in a person's identity and/or lived experience." (van Anders et al., 2019, p. 9).

The above definitions and uses of "identity" in relationship to gender are confusing. What exactly is a deeply felt sense of self as boy, male or man, or girl, female, or woman? Many people think this sense resides in the brain. But if so, where? And what, in a material sense, would it be? A specific structure? A set of interacting neurons? And is "inherent", as used in the APA definition, something that just appears at the right age, as suggested by HealthyChildren.org? If, as van Anders et al. (2019) stated, trans identities (the authors of this essay see trans as a possible type of gender identity) vary among trans individuals, does that mean that a particular gender identity such as trans is not inherent? Or are there several types of trans identities each of which is inherent? Although for many, it is a grounding belief that everyone has a gender identity, agreeing on what is meant by those two words remains problematic (Byrne, 2019).

It is hard to construct a workable definition of gender/ sex identity if one does not understand how, in general, individual identity develops. Within developmental psychology, predominant theories of gender and development have produced many observations and experiments leading to a developmental timeline for the emergence in children of self-knowledge as boy or girl. But because these predominant developmental theories conceptualize identity as a "thing", a fixed trait that presumably resides somewhere inside the brain, they cannot accommodate the current era in which gender-related identities seem to be anything but fixed. In this article, we develop an alternative model, based on the idea that identity is a dynamic process rather than a fixed trait. The 
process of identity is sustained within the body as part of an individual's existence within a social matrix (Haslanger, 2012). We focused on the first three years after birth, that is from a neonate who cannot articulate or who does not otherwise evidence a sense of maleness or femaleness, to a toddler who has developed the ability to self-label as boy or girl and who expresses preferences for objects and play patterns that have, within whatever culture the child is living, recognizable gender-specific valences.

Our essay has the following structure. First, we will discuss and introduce the recent terminology of gender/ sex and embodied gender/sex identity. Second, we will introduce dynamic, process models for the formation of embodied gender/sex identity. Third, we provide evidence that has the potential to link the development of embodied gender/sex identity to sensori-motor development. We present data that demonstrate that mothers may handle, speak to and physically interact differently with infant sons compared to infant daughters. Our essay will conclude by offering a model to frame these data (and other published work), and to draw a roadmap for future empirical and theoretical studies.

\section{New Terminology}

\section{Gender/Sex}

Over the past several decades, the terms "sex" and "gender" have been used with a variety of meanings. In their textbook Gender Development, Blakemore, Berenbaum and Liben (2009) discussed some of these. For the purposes of their text, they concluded that using "sex" "to refer to sexual behavior and sexuality...and clear biological phenomena (e.g. sex hormones, sex chromosomes) is essentially universal" (p. 3). They noted as well their consistent use of the terms "gender roles", "gender stereotypes" and "gender identity". In their discussion they said that they would usually label behavioral differences between boys and girls as "sex differences", making clear in doing so that they did not intend to downplay the importance of social or cultural forces. "On the contrary," they wrote, "...many factors influence gender development: biological, cognitive, social, and cultural" (Blakemore et al., 2009, p. 3).

As Blakemore et al. (2009) indicated, the sex-gender terminology has served two purposes. On the one hand, dividing a term such as gender into subcategories such as roles or stereotypes has facilitated the design of studies aimed at more precisely describing gender as a cultural phenomenon. On the other hand, the terms sex and gender also imply a causal model in which "many factors" influence gender development. The implied model is often additive (biology plus culture) although additive models may include "interaction" as a third, poorly articulated term. Unger and Crawford (1993) pointed out the difficulty with the sex versus gender terminology when they wrote "With the possible exception of very specific reproductive behaviors, however, it is not possible to determine how much of a particular trait or behavior is influenced by biological versus social factors... Complex interactions are the rule, not the exception" (Unger \& Crawford, 1993, p. 124).

Responding to such conceptual difficulties, in a research project that focused on hormones, which are most often listed as a feature of "sex", van Anders and Dunn (2009) introduced the term gender/sex "because", they wrote, "differences cannot knowingly be attributed to biology or gender socialization" (p. 207). van Anders (2015) defined gender/sex as pertaining to "whole people/identities and/or aspects of women, men and people that relate to identity and/or cannot really be sourced specifically to sex or gender" (Table 2 in van Anders, 2015). Fausto-Sterling (2012) and Pitts-Taylor (2016) used the term sex/gender to connote body-based characteristics that are shaped by gendered social interactions. However, to maintain consistency with the way the term was initially introduced by van Anders and Dunn (2009), we have adopted the term gender/sex.

The gender/sex terminology is gaining currency within the field of psychology. Hyde, Bigler, Joel, Tate, and van Anders (2018) also urged the adoption of gender/sex, in recognition of both the practical impossibility of separating sex from gender, and as a heuristic to help researchers to move beyond the conceptual bifurcation of sex and gender. In this essay, we use the term gender/sex unless we refer to the language used by authors we cite, in which case we respect their original usage. To date, the idea of gender/sex has referred only to phenomena observed in cross-sectional studies. In this essay we apply the gender/sex terminology to longitudinal studies with the hope, eventually, of seeing how gender/sex characteristics of a particular age group emerge from preceding experiences.

\section{Gender/sex is embodied}

We conceive of gender/sex as embodied and distinguish embodied gender/sex from social roles or stereotypes. Such roles and stereotypes are often thought of as cultural beliefs and appropriate behaviors taught to children through a process of socialization. We use the term "experience", rather than "socialization", to include vocal interactions, touch, affect, visual input, smell and dyadic interactions 
between the infant and caregiver (Thelen, 2000a; FaustoSterling, Crews, Sung, García Coll, \& Seifer, 2015). This understanding of experience is well known to students of behavioral development in animals (Gottlieb, 1997; WestEberhard, 2003). If, in infancy, sensory experiences are a primary path of gender/sex socialization, then gender/sex, as part of a system mediated by neuro-sensory information, cannot be described separately from the developing body.

The idea of "throwing like a girl" provides an illustration. Do girls throw a certain way because of aspects of shoulder and torso anatomy (i.e., is it caused by innate biological differences between the sexes?) or is a supposedly girlish throwing stance culturally derived (i.e., is it an epiphenomenon of gender)? In fact, it is both biological and cultural. Once developed it is in the body, a set of reflexes that conditions each step in the throwing process. By analogy, a touch typist accustomed to an English QWERTY keyboard can with difficulty and many rounds of practice overcome the stumbling blocks imposed by the small differences found on a keyboard adapted to the accented letters found in Portuguese or Spanish. In similar fashion, a person can consciously unlearn the neuromuscular steps that constitute girlish throwing. Because throwing like a girl is a biological effect of a cultural preference, practice, and developmental experience, and because it is wired into the body as a strong attractor (i.e. it is difficult to change), we refer to it as embodied gender/sex (Downey, 2009; Young, 1980, 1998).

\section{Gender/sex Identity}

Until recently, developmental psychologists defined gender identity as an inner or personal sense of self as male or female (Huston, 1983; Ruble, Martin, \& Berenbaum, 2006). How, though, does this sense of self develop, and where, exactly is it located? In order to self-label, infants and toddlers must take stock of their world and categorize gender outside of themselves. Once they have categories, they can figure out where they fit. But based on what? If not their genitals (at first), then to figure out their own location they must absorb data from how adults touch, hold and move them, speak to them, dress them, style their hair, etcetera. In other words, external categories must exist for an internal identity to develop. There is a back and forth between the body's interior which presumably involves perception, the establishment of neural and sensorimotor networks, and memory circuits in the central, peripheral, and autonomic nervous systems and the social world in which an individual child finds itself. It is this interplay between the exterior world and internal physiology that we call "gender/sex identity".

\section{Gender/sex Identity Is Embodied}

In Gender as Soft Assembly (Harris, 2005) psychologist

Adrienne Harris observes that gender (her usage) emerges "from the immersion of an infant, with only a small repertoire of capacities, in an immediately complex interaction with a social, physical world" (p. 150). Harris wrote that we do not need a concept of core gender identity. Although gender emerges as a "patterned, complex self-state" (p. 150), it is idiosyncratic. It differs from one individual to the next because individuals have different bodies through which they differently experience varying conditions of development. Instead of a core, she posits a process which, despite some regularities, remains individually unique. We agree that there is no such thing as a freestanding, inherent, internal core gender identity. Instead, we propose that embodied gender/sex identity begins as a set of processes by which newborns, who start out with limited physical and cognitive abilities, awash with stimuli from the physical environment and from adults (who themselves respond to the assumed meanings of the baby's external genitalia), react bodily and cognitively to the sensory input. This input, in turn, influences the baby's physical and cognitive capacities. Such capacities promote the infant's increasing independence and as the infant develops into a toddler, allows the child to incorporate into itself (and apply to others) the gender/sex-related information it has distilled. This process of embodied gender/sex identity continues throughout the life cycle, although in this essay we focus on the period from infancy through toddlerhood.

\section{Modeling Identity: Fixed Trait Versus Dynamic Process}

In this section we review previous developmental approaches to the study of identity formation. We first consider a large literature which, at its heart, understands gender/sex identity to be a trait, or property of an autonomous individual. We then compare this literature to co-existing accounts of identity as a continuously sustained intersubjective process, evidenced in individual bodies and behaviors. Dynamic theories view identity as a set of interdependent processes involving individuals' continuous engagement with their social and physical worlds. We conclude this section by delving more deeply into some of the underlying assumptions of gender/sex formation when looked at through the lens of "thingness" compared to the lens of process. 


\section{Developmental Psychology and Gender/sex Formation}

In an article entitled "Sex-Typing", Huston (1983)

reviewed and organized a substantial body of workstemming primarily from the 1970's and 1980's-- on the development of sex-typing in childhood. Huston used a $5 \times 4$ matrix to aid in understanding the complex strands of behavior, culture and biology that she thought contributed to sex-typing. On the horizontal axis (rows) she aligned four constructs-concepts or beliefs, identity or self-perception, preferences, attitudes or values, and behavioral enactment. On the vertical axis (columns) she listed five areas of content: "biological gender", "activities and interests," "personal-social attributes," "gender-based social relationships," and "stylistic and symbolic content." To get a sense of her system, consider the content area in row 1 that she labeled "biological". Reading across Row 1 , under Column A, one finds gender constancy as the relevant concept or belief. Under Column B, "Identity or self-perception," one finds "gender identity" (which Huston defined as an "inner sense of maleness or femaleness"), and "sex role identity" (which she defined as "self-perception of interests, abilities"). Under column C ("preferences, attitudes, values,") she listed the "wish to be male or female," and in Column D ("Behavioral enactment,") Huston listed behaviors such as "displaying bodily attributes of one gender..." (Huston, 1983, p. 290).

Fifteen years later, Ruble and Martin (1998) published the review article "Gender Development". The title reflected changes in language use concerning sex and gender. By 1998, and into the present, a separation had emerged between the word "sex" to mean something of strictly biological origin, or "gender" to mean a culturally transmitted trait. Ruble and Martin (1998) continued to use Huston's basic framework but added a 6th content area (Row) that they called "values". They also dropped the term "sex-typing" in favor of "a matrix of gender-typing." Whereas Huston named the first content area "biological gender," Ruble and Martin (1998), in keeping with efforts to use "sex" to indicate biology, renamed this area "biological/categorical sex." In agreement with Deaux (1993), they used the term "sex" to designate a genital or demographic category, while applying the term "gender" to stereotypes, concepts, or beliefs about human male or female preferences or behaviors.

Ruble and Martin (1998) constructed a timeline for gender development: at about 12 months infants could respond to gender-specific cues, by 2.5 years most could label themselves as male or female, and between 2.5 to three years children began to evidence a rudimentary understanding of gender stereotypes, gravitating more often to toys that stereotypically matched their self-label (cars for boys, dolls for girls). During their third year, children came to see gender as a stable trait, and subsequently their knowledge of gender-specific items and behaviors increased markedly. Ruble and Martin referred to this as "complete sex category constancy" (p. 963).

Eight years later and reflecting the rapid accumulation of studies on gender development, Ruble, Martin, and Berenbaum (2006) produced a new review in which they discussed the possible causes of developmental change, as seen through the lenses of biology, cognitive development and socialization theory. The authors explicitly framed this new review as a normative account of development. For example, in a section entitled "Variations in Core Gender Identity" they concentrated on "research concerned with the etiology of core gender identity and its disorders" (pp. 862-863; emphasis added). Throughout the article, the authors cited normal ranges of continuous variables such as hormones. The words normal or normally appear 18 times, indicating a binary view of development in which the categories of normal and disordered were seen as distinct. Evidence that words such as "disorder", "normal" and "normativity" carry theoretical implications can be found in Martin and Ruble (2010). In contrast to their earlier reviews, in this publication they focused on patterns and processes in gender development and in keeping with the theories discussed in the 2010 review, the words "disorder", "normal" and "normativity" did not appear in the main text (Martin \& Ruble, 2010).

Throughout their reviews Huston (1983), Ruble and Martin (1998) and Ruble et al. (2006) interlaced the terms "sex" and "gender" with the concept of gender identity. Huston (1983) cited Kohlberg's view that gender identity "is a self-categorization as boy or girl, which then serves as an organizer of incoming information and attitudes" (p. 397). Ruble and Martin (2006) added an affective componentwhether a person feels they are male or female. They reviewed studies on non-clinical populations to delineate the timing of gender identity appearance and used studies of clinical populations to assess the etiology of gender identity formation. Judging from Ruble and Martin's (1998), Ruble et al.'s (2006), and Huston's (1983) matrices, all four authors concurred that gender identity had social as well 
as biological components. According to Huston, Kohlberg thought that self-identity formed the basis for subsequent social preferences for male or female toys, companions, and clothing, although subsequent research on the timing of certain preferences, relative to the timing of self-knowledge about gender, has been contentious (Bussey \& Bandura, 1999; Martin, Ruble, \& Szkrybalo, 2004).

Although the data advanced by the above debate are critical to understanding the process of gender identity development, a challenging problem remains. This is evident if one examines Money and Ehrhardt's (1972) definition of gender identity (taken from original work that preceded Kohlberg, 1966) as "the private experience of gender role" and gender role "as the public expression of gender identity" (Money \& Ehrhardt, 1972, p. 284). In other words, the public and the private, the inner feeling and the exhibited behaviors are the inseparable faces of a single coin. While this presents most developmental psychologists with a "chicken and egg" type of problem, process approaches to gender/sex emergence; embrace the inseparability of public and private, of inner feelings and exhibited behaviors.

\section{Studying Gender/sex as a Dynamic Process}

In contrast to the gender development literature, no one has neatly wrapped up studies of gender/sex development as a set of relational processes into four comprehensive review articles. Yet there is a significant literature on the topic. Fausto-Sterling (2019) argued that the tools to study gender/sex identity formation derive from the intersection of three types of inquiry. (1) Beebe and her colleagues focused on the importance of the intra-dyadic dynamics of mother and infant, specifically the importance of "arousal, affect, space and time in the early organization of experience" (Beebe, Lachmann, \& Jaffe, 1997, p. 134).

(2) Dynamic systems researchers have established that cognitive development is embedded in a physical world, and that motor development is a critical aspect of emotional and behavioral development (Smith, 2005; Smith, Thelen, Titzer, \& McLin, 1999; Spencer, Smith, \& Thelen, 2001; Smith, Jayaraman, Clerkin, \& Yu, 2018). (3) Thompson (2007) and Thompson and Varela (2001) applied the concepts of embodied cognition and phenomenology to human development, providing insights applicable to gender/sex development.

\section{Intradyadic Dynamics}

Building on the work of Daniel Stern (1985), psychoanalysts Beatrice Beebe, Frank Lachmann and colleagues connected interactions within mother-infant dyads to the subsequent quality and degree of infant and toddler maternal attachment (Beebe et al., 2010; Beebe \& Lachmann, 2002; Stern, 1985). Recording the second by second behaviors (alone and in mutual response) of the individual contributors to the dyadic unit (usually mother-infant), and using a longitudinal study design, these researchers asked how infants organize and represent to themselves their experience of their primary caregiver (Beebe et al., 2000; Beebe \& Lachmann, 1994). During the first year, they proposed, the infant develops presymbolic internal representations of self and object. These presymbolic formations form the foundation for an infant's emerging ability to use symbols to represent itself and surrounding objects. Beebe and Lachmann (1994) "assume that representations in the first year are encoded in a nonverbal, imagistic, acoustic, visceral, or temporal mode of information and that they may not necessarily be translated into linguistic form." (p. 132). As development proceeds, early representations form the scaffolding for subsequent experiences, leading to a representational restructuring that by 18 months takes on symbolic forms.

The interactive structures Beebe and colleagues describe are neither causal nor symmetrical. Rather, they are based on the expected probability that a particular response or behavior from one dyadic partner will elicit a particular response or behavior from the other. Beebe and Lachmann (2002) highlighted four aspects of dyadic interactions-- state transforming (an expectation that one partner can change a state of arousal of the other--for example, a mother bouncing a baby on her lap to get her to stop crying), facial mirroring (the expectation from one partner that the other will mirror an offered affective signal-for example, a father leaning over an infant and smiling broadly in the hope that the infant will return the smile), disruption and repair, (an expectation of how easily and quickly a dyad can repair a facial-visual mismatch-for example, a caregiver and infant lock eyes, the infant breaks eye contact, and the caregiver leans in to try to re-establish eye contact) and interpersonal timing, (expectations of the extent of vocal rhythm matching and interruption). According to Beebe and Lachmann (1994) these interactions structure the internal organization of dyadic experience, which, in turn, shapes the growing capacity for self-regulation and choreographs dyadic interplay during the first postnatal year.

\section{Dynamic Systems and Motor Development}

Dynamic systems researchers who study infant development argue that motor skills provide an important 
gateway to cognitive and emotional development. Thelen emphasized "the continual bombardment of real-life, multisensory but coherent information that fuels the engines of developmental change as infants learn to act in social and physical worlds." (Thelen, 1995, p. 90). We theorize that early sensorimotor interactions within infant-caregiver dyads generate gender/sex-differentiated behaviors that in turn provide "a variety of perceptualmotor experiences" that infants retain differentially and weave into a developing gender/sex identity. For example, Fogel et al. (1997) described the recruitment of social meaning to a neural reflex and the subsequent sensorimotor transformation of that reflex. Feldman (2015) and Feldman, Masalha, and Alony (2006) studied the dynamic interactions between caregiver and child that shape the emergence of self-regulation and emotional capacity during the first 10 years of life. Although none of these researchers looked specifically at gender/sex, the dynamic processes by which social meaning becomes embedded in inter-neural and neuro-muscular connections ought to be applicable to dyad interactions that are differentiated by infant and parental gender/sex.

Dynamic systems researchers emphasize the study of individual variation, not just aggregated group statistics; they employ the same type of second by second analysis found in studies of intra-dyadic interactions, and they have a mantra which demands longitudinal studies. That mantra is: when examining a phenomenon, look first at the time period before it is measurable, record details of the phenomenon during the period of emergence, and finally, describe and account for the phenomenon's emergence and stabilization as a consistent and measurable skill or personality feature. We wish to apply this mantra to the study of the process of gender/sex identity.

Thelen et al. (1993) and Spencer et al. (2000) illustrate this theoretical and experimental approach. Thelen and her colleagues observed four infants weekly, from 3 to 30 weeks, and biweekly thereafter, until 52 weeks, as the infants learned to reach and grasp a toy. The researchers described a suite of components (e.g. head and torso control, and the ability to touch and grasp nearby objects) needed to achieve stable reaching. During the period that preceded a transition to reaching, each child differed in terms of the timing and order of appearance of specific behaviors. All four infants used different motor strategies as they attempted to reach for a desired toy. Two had large and strong spontaneous movements and damped these down to achieve their goal. In contrast, the others, who were quieter, ramped it up, producing faster and more energetic efforts to lift their arms. Thus, each child accomplished a goal of reaching for a toy by accommodating their individual motor inclinations. By analogy, as infants develop into toddlers, they use individual methods to classify the myriad objects and behaviors that surround them, including discovering gender/sex categories; as time passes, they follow category discovery by figuring out and embodying category belonging. The concepts provided by theories of embodied cognition enable a next step-to conceptualize the application of intra-dyadic dynamics and the dynamic systems of motor development to the study of gender/sex.

\section{Embodied Cognition}

Thompson (2005) employed "an enactive approach" according to which the mind is dispersed throughout the physiologically functioning body, which, in turn, is embedded in the world. Thus, the sense of self does not collapse into structures inside our head. Instead, mental life emerges from three interlinked modes of bodily activity: "self-regulation, sensorimotor coupling and intersubjective interaction". (Thompson, 2005, p. 408). Thompson distinguished between an unconscious "body schema" and a conscious "body image." The latter is what one contemplates when, for example, looking in the mirror. The former, which encompasses an interior sense of gender/sex or orientation, is neither intentional nor conscious. Rather, Thompson defined it as "an integrated set of dynamic sensorimotor principles that organize perception" (p. 411).

Varela $(1996,1997)$ and Thompson and Varela (2001) proposed that an individual's consciousness emerges via a process of radical embodiment. In opposition to standard neuroscience that looks for "the neural correlates of consciousness", they proposed that consciousness emerges within and between the interactions of the brain, body and world. We use Varela and Thompson's ideas in our account of the development of gender/sex identity, described more fully in the final section of this essay. Briefly, we propose that an infant initially absorbs bodily informationincluding information about gender/sex-- within the dyad. Gradually, however, the infant's newly embodied (sensorimotor, regulatory and so on) information and skills promote autonomy, i.e. separation from the dyad. Thus, the individuating infant already has rudimentary elements of gender/sex woven into his or her neuro-sensory repertoire. 


\section{Comparing Models and Assumptions}

Philosophers of biology John Dupré and Daniel Nicholson (2018) urged "that we abandon a theory of things in favor of one centered on processes" (p. 1). Processes, they argued, appear stable at any particular location or timescale. In a compatible discussion, Overton (2014), writing specifically about psychology, compared a Cartesian-split-mechanistic worldview to a process-relational one. He illustrated the process-relational side by citing the traditions of embodied, dynamic, transactional, and psychobiological systems developed in varying forms by Piaget, Lerner, and Liben. Other particularly good examples include Thelen and Smith (1994), Diamond (2007) and Gottlieb (1996; 1997).

In Table 1 we compare a theory of things to a process theory of identity. Depending on the type of theory, gender/ sex identity has different characteristics and origins. Those who rely on a theory of things conceptualize gender/sex identity as a fixed trait that appears in toddlers. The trait is understood as the property of an autonomous individual. As evidenced by a substantial research literature that searches for brain differences between men and women, cis and trans, or gay and straight individuals, a commitment to "thingness" is often linked to the idea of a fixed brain location for identity (Joel \& Mccarthy, 2016; Joel et al., 2018; Manzouri \& Savic, 2018; Swaab \& Hofman, 1995; Sadjadi, 2019). That commitment goes hand in hand with the search for autonomous root causes-for example specific genes or a shout out to prenatal hormones (Bailey et al., 2016; Jordan-Young, 2010).

Table 1. A comparison of two theories of identity

\begin{tabular}{ll}
\hline Theory of things (traits) & Process (dynamic) theory \\
Identity is: & Identity is: \\
\hline A fixed trait & A dynamic process \\
Static-hard wired & Changeable-softly assembled \\
$\begin{array}{l}\text { Individual-autonomous } \\
\text { A "thing" located in the brain }\end{array}$ & $\begin{array}{l}\text { Felt/maintained continuously } \\
\text { throughout the body }\end{array}$ \\
$\begin{array}{l}\text { Produced by } \\
\text { autonomous/biological } \\
\text { processes }\end{array}$ & Produced by stable interactions \\
$\begin{array}{l}\text { Felt subjectively } \\
\end{array}$ & Felt subjectively \\
\hline
\end{tabular}

Process theories offer contrasting points of view. While emphasizing stability, they allow for change or modulation throughout the life cycle. Process theorists emphasized a continuous interplay between autonomy and intersubjectivity (Higgins, 2018; Legrand, 2006), and considered that the biological components of identity are dispersed in the body via peripheral and autonomic as well as centralized physiological systems. The one thing that both theories hold in common is that identity, in the end, is experienced as a subjective state.

\section{Constructing a Timeline}

Gender/sex consists of levels of organization from the molecular to the anatomical to the behavioral. Relevant processes occur on timescales that range from nanoseconds (in the case of a stochastic change in molecular conformation that affects a particular gene's expression), to milliseconds (in the case of nerve impulses that produce a bodily response as rapid as an eye-blink), to the days, weeks and even years that axons and dendrites may require to grow and connect as they stabilize networks of neural transmission. Gender/sex may include anatomical changes during the life cycle (e.g., genital growth during puberty and hormonal changes during aging). Lastly, gender/sex may also be understood to involve a timescale of many generations via evolutionary changes. In this article, we do not discuss the latter, but want to acknowledge that the process of sex includes this multi-generational time scale.

In Figure 1 we summarize critical features of current knowledge about gender/sex processes during the first three years of development. At birth (or likely prenatally during the last trimester) (see Moon, Lagercrantz, \& Kuhl, 2013), the infant nervous system records sensorimotor information. We adopt Beebe and Lachman's (1994) use of the term presymbolic to refer to representations "encoded in a nonverbal, imagistic, acoustic, visceral, or temporal mode of information" (p. 132). As drawn in Figure 1, the gender and development literature suggests a phase shift to symbolism between 15 and 18 months, a period that roughly parallels the emergence of language skills. During the period from 18 to 36 months toddlers or young children consolidate and stabilize gender/sex self-knowledge and gender/sex knowledge of the world. They often express this knowledge symbolically-via a pink/blue color scheme and clothing or play preferences (Eichstedt, Serbin, PoulinDubois, \& Sen, 2002). In the next several sections of this 
A.

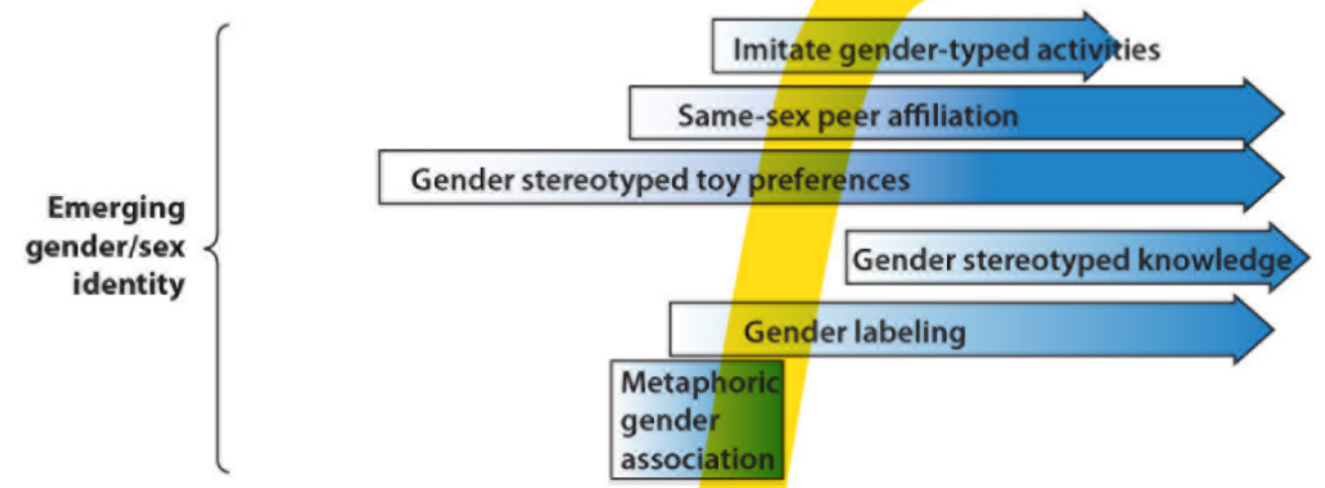

B.

C.

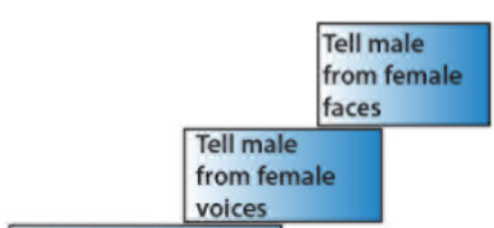

Different patterns of

face to face

communication on

$\mathrm{m} / \mathrm{s}$ and $\mathrm{m} / \mathrm{d}$ dyads

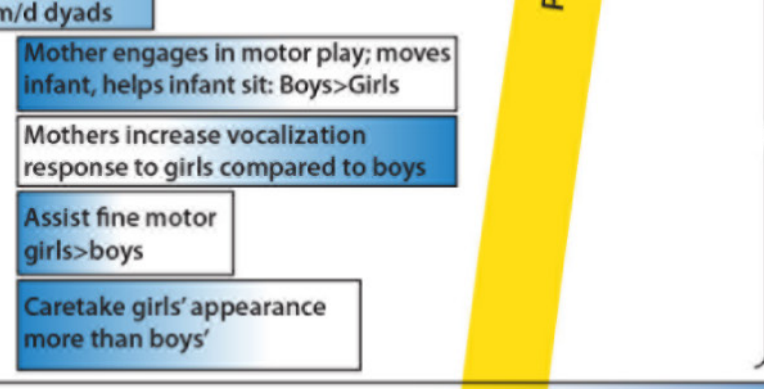

\begin{tabular}{|ll}
\hline Connectome efficiency sex differences & ?(endpoint unknown) \\
\hline Modular to inter-modular connectome structure & ? (endpoint unknown) \\
\hline
\end{tabular}

\begin{tabular}{|l|}
\hline Prefrontal cortex \\
\hline Parietal and temporal association cortex
\end{tabular}

\begin{tabular}{|ll|}
\hline Myelination & ? (endpoint unknown) \\
\hline Synaptogenesis and synaptic pruning & ? (endpoint unknown) \\
\hline
\end{tabular}

Presymbolic gender/sex

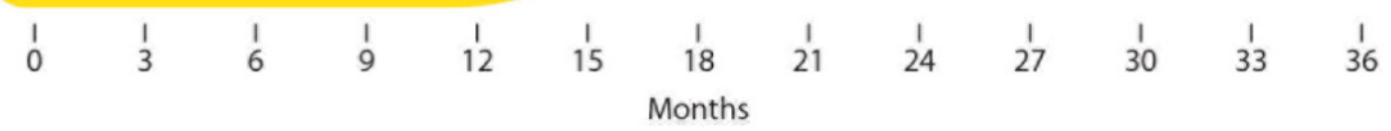

Figure 1: Composite timeline. Illustrates systems undergoing change from birth to three years. Longitudinal lines show gradients of activity, from light (low activity) to dark (high activity). See main text for further explanation. 
essay, using Figure 1 as a guide, we selectively review relevant physiological, dyadic and autonomous behaviors that mark the presymbolic, transitional, and symbolic phases of gender/sex development. We remind the reader that we draw on a literature that comes almost exclusively from studies of white, middle class, European-origin families and that patterns of gender/sex-related behaviors and interests discussed here are not universal (Lew-Levy, Boyette, Crittenden, Hewlett, \& Lamb, 2019).

\section{Neural Processes}

Eliot (2018) wrote, "Toy play may look instinctive in children-as when we see toddlers cuddling a doll or pushing a toy truck across the floor-but every piece of such actions requires learning and tuning of neural circuits to the specific sensory, motor, spatial, social, cultural and motivational demands of both object and environment" (p. 171). To explore Eliot's remark, we selectively review findings from the neurosciences that we believe to be relevant to the presymbolic recording of gender/sex. (See also Figure $1 \mathrm{C}$ )

Even before birth, synaptic connections involved with sensory (i.e. sight, sound, smell, touch, taste) and related motor (involved with movement that responds to and controls sensory input) activities proliferate exuberantly. As neurons attain peak bushiness after birth, they gain specificity by pruning some connections and strengthening others. Nervous transmission also becomes more efficient as the long nerve fibers, that reach, say, from the fingertips to the cerebral cortex, gain electrical insulation via myelination. As represented in Figure 1C, nerve cell branching and development is especially active during the first six months in the sensorimotor,prefrontal, parietal and association cortices (Thompson \& Nelson, 2001), while intense myelination occurs during the first year of infancy. Critical to the idea that gender/sex-at least initially-is a process requiring both dyadic interactions and interactions between the infant and objects in its world is the fact that specific synaptic connections form under the influence of specific experiences. Writing about adolescent brain development, Casey, Tottenham, Liston and Durston (2005) presumed that a change in brain structure and function is "an experiencedriven maturational process that reflects fine-tuning of neural systems with experience and development" (Casey, Tottenham, Liston, \& Durston, 2005, p. 108).

We know little about the appearance of gender/sex differences in the central nervous systems of infants. Giedd and colleagues documented differences in brain structure between boys and girls as young as four years old (Giedd, Castellanos, Rajapakse, Vaituzis, \& Rapoport, 1997; Gogtay et al., 2004; Lenroot et al., 2007) However, to date a dynamic account of gender/sex and neural development during the period of presymbolic learning does not exist.

There is, however, the beginning of a story to tell about gender/sex development and the connectome. Cao, Huang, and $\mathrm{He}$ (2017) defined the connectome as a complete set of neural elements--cells, collections of interwired cells (nodes) and the linkages between nodes. They further defined a module as a set of densely linked nodes that are more tightly connected to each other than to the larger network. The latter facilitates global communication between different brain regions. During fetal development, the number of modules increases with time. After birth, modularity decreases, and inter-module integration best describes developing brain topography. Stated another way, short-range connections seem to dominate the fetal brain. Long range inter-modular connections develop primarily after birth, showing up clearly in one-year olds. As they strengthen in two-year olds, brain networks become more globally integrated. Cao et al. referred to this as a "remarkable reorganization, resulting in the transformation of the connectomic architecture from a relatively randomized configuration to a well-organized one" (Cao, He, et al., 2017, p. 495).

Studies relating gender/sex to the developmental connectomics of fetal, preterm, infant and toddler brains are still in their early days. To date, one publication reported that there are no differences between males and females in the efficiency of either the global or local information transfer in two-week olds and one-year olds, the time frame we have designated for presymbolic formations. However, both local and global brain network efficiency is significantly greater in male compared to female two-year olds (Yap P-T et al., 2011), the time point that marks the end of our proposed phase transition to symbolism. Thus, it appears that gender/sex differentiation of the connectome begins during (and possibly is a driver of?) the transition period. Yap et al. did not speculate on possible mechanisms leading to this divergence. Elucidating the general and specific drivers of this connectome transition awaits further exploration.

\section{Dyadic Behaviors and Presymbolic Gender/sex Skills}

During the presymbolic period a number of gender/sexrelated dyadic behaviors and infant skills emerge. We list 
some examples in Figure 1B. Fausto-Sterling, García Coll and Lamarre (2012) offered a more complete accounting. In the empirical section of this essay we offer and analyze evidence that especially from months three to six, mothers engaged in more gross motor activities with sons than with daughters. Fausto-Sterling et al. (2015) reported that from months three to six, mothers of daughters more frequently adjusted a daughter's (compared to her son's) appearance by combing her hair, straightening her dress or repositioning a hair ribbon or barrette (Fausto-Sterling et al., 2015).

During this same time period mothers vocalize more to daughters than to sons (Sung, Fausto-Sterling, Garcia Coll, \& Seifer, 2013). By six months the infants themselves start to recognize gender/sex in their world. As indicated in Figure $1 \mathrm{~B}$, they start to distinguish male from female voices and faces, and by 12 months they start to associate male voices with male faces and female voices with female faces. We hypothesize that gender/sex differentiated differences in dyadic behavior shape the underlying developing nervous system and produce the ability to recognize aspects of gender/sex (what we refer to as gender/sex skills) in the infant's world. We call these interconnecting processes "presymbolic gender/sex formations".

\section{Statistics and Chunks: Presymbolic Input Becomes Embodied via Touch and Movement}

\section{The Infant as Statistician}

Presented with repeated and diverse sensory inputs, infants measure the frequencies of sequences of motor, visual, object and linguistic events, extracting "chunks", i.e. elements that co-occur, which they store in distributed neural networks. As they reencounter similar chunks, the co-occuring elements become more tightly linked, even as they evolve with the child's age and skill levels. It is through these general learning mechanisms, we hypothesize, that infants extract and stabilize the structures and meanings of gender/sex, first presymbolically, and then via language and symbolism (Balas, Saville, \& Schmidt, 2018; Gliga, 2018; Mareschal, French, \& Quinn, 2000)

To illustrate, we recount two early sequences of dyadic (mother-daughter) interaction. We present the details of videotaping in the Methods section of this article. Watching videos recorded during in-home visits when the baby was 2.4 and 3.2 months, we see the mother washing her child's head. Throughout each two- to four-minute episode, the mother gently narrated her actions. She encouraged the baby to enjoy the wash, saying "doesn't that feel good? Do you want to help?" and, as she massaged the soap into her head, "Oh you smell so good." The baby smiled and tried to participate, which the mother encouraged. In this chunk the infant experienced a combination of what appeared from her reaction to be pleasurable tactile sensations on her head with a maternal narration of events. At 3.4 months, a new element appeared, when the mother decided, during changing and dressing, that the baby's hair was long enough to brush. She brushed gently for 13 seconds and said "that's not bad. All done. All done. That's pretty." The baby, who was sitting on the changing table, had been looking around the room, but as the mother said the first "All done", she looked directly up at her mother's face as the mother bent over her. This was a second chunk in which again, there was a pleasant tactile sensation on the head, this time in combination with positive dyadic contact and maternal patter about how pretty the baby looked. It seems likely (although this warrants empirical study) that the second chunk built on, or interacted with and strengthened the first, while for the first time (in our observations) a gendered comment ("that's pretty") became part of the event network.

What an infant sees, hears, touches and is touched by changes with development. At three months, an infant has few motor skills, and the adult caregiver controls most sensory input, either directly or by controlling the environment. As Smith and colleagues so wonderfully demonstrated using infant head cameras, at three month's an infant pretty much only saw close-ups of adult faces and chests while experiencing tactile and auditory input (Byrge, Sporns, \& Smith, 2014; Clerkin, Hart, Rehg, Yu, \& Smith, 2017; Smith et al., 2018). But as the infant crawls and then walks, the visual world opens up to them. Physical autonomy, in other words, widens the infant's world (Campos et al., 2000).

\section{Neuromuscular Development Meets Culturally Structured Patterns of Infant Care}

Nishiyori et al (2016), examined the activation of motor cortex neurons as infants acquired motor skills such as reaching and stepping (Nishiyori, Bisconti, Meehan, \& Ulrich, 2016). As predicted by developmental systems theorists (Gottlieb, Wahlsten, \& Lickliter, 1998), unskilled infants call on a redundant repertoire of neural circuits. As they gain experience through increasingly goal-directed activities, the initially large areas of neural activation become more restricted and refined. Thus, Nishiyori and colleagues (2016) argued that the neural responses that underpin specific motor activities derive from both the specific goal and the 
experience of pursuing it. Work on multisensory, multimodal processing echoes this general idea that infants process sensory input broadly in early development, but as they gain both sensory and symbolic experience, multi-sensory perception narrows and becomes more culturally specific (Murray, Lewkowicz, Amedi, \& Wallace, 2016) Work on the developing connectome points to a similar theme: the increasingly complex connections established between neural modules during the first year of development (Cao et al., 2017).

The multisensory systems studied by Murray et al (2016) and by Lewkowicz and Ghazanfar (2009), Lewkowicz, Schmuckler and Mangalindan (2018), Kuhl, Tsao, Liu, Zhang, and De Boer (2001), and Kuhl et al. (2006) exemplify the development from diffuse to focused processes. This body of work involves connecting faces to specific vocalizations and language recognition. Studies suggest that both before birth and for the first three to six months after birth, infants exhibit broad, low level responses to sound and sight stimuli. Over time, responses narrow. At first, an infant may respond fully to a non-native spoken syllabic sound or associate a monkey grunt with a monkey's face; with further sensory experience, the response narrows and becomes native language and species specific. Lewkowicz and Ghazanfar (2009) suggested that this perceptual narrowing results from the selective elaboration of synapses in specific response to postnatal experience and Lewkowicz et al. (2018) demonstrated that between the ages of four to five and eight to 12 months, infants develop the abilities to "perceive, learn, and generalize recursive, hierarchical, pattern rules" (p. 1). Does the infant response to gender/sex follow this pattern--broad and inclusive at first, followed by a narrowing introduced by gender/sex specific experiences? The non-invasive neuroscience techniques currently available for infant study (Kuhl, 2010), could be incorporated into the study of dyadic interactions, perhaps offering a neural window through which to infer answers to this question.

As neurologically complex as they are, the multisensory tasks of connecting visuals of faces to the sounds that emerge from them, or of discriminating between native and non-native spoken sounds, seem simple in comparison to the task infants face of identifying and embodying gender/sex. Evidence from the literature on gender and development suggests, however, that we can fruitfully use the general principles gleaned from these simpler cases to narrate the acquisition of embodied gender/ sex identity. This line of thought provides a framework to structure future empirical investigation. We understand our own work, including the data presented in the empirical component of this essay, to be a "proof of principle", a jumping off point for the design of dynamical, multisensory approaches to the study of gender/sex and development.

\section{Symbolic Gender/sex}

As illustrated in Figure 1, between approximately 15 months and three years of age, a phase shift, followed by gender/ sex identity stabilization, occurs as the infant transforms presymbolically embodied gender/sex knowledge into symbolically embodied gender/sex identity (see discussion by Fagot \& Leinbach, 1993). The consolidation of gender/ sex in individual children has been thoroughly reviewed as a progression from gender self-awareness to gender constancy, and finally to the association of genital knowledge with gender identity (Ruble et al., 2006). By the time children enter nursery school, kindergarten and elementary school, gender/sex behaviors and selfknowledge have become well-established (Fabes, Martin, \& Hanish, 2003; Fabes, Martin, Hanish, Anders, \& MaddenDerdich, 2003; Thorne, 1993). In a study that illuminates the phase transition we propose in Figure 1, Zosuls et al. (2009) correlated the emergence of gender-typed play with a child's use of gender labels. They suggested that "knowledge of gender categories might influence gender typing" during this transitional period (Zosuls et al., 2009, p. 688)

Even before language emerges, we saw signs of embodied gender/sex. An example from our videotapes (described in the Methods below) illustrates. In Figure 2, we present drawings derived from still frames of the same girl described above while being bathed and dressed at 2-3 months-now aged 15 months-- as she explored a walk-in playhouse she had just received for Christmas. The house has a mauve door, yellow siding, pastel green shutters and a blue roof. A rocking horse with the same pastel shades of pink and green stands outside. In this roughly eight-minute sequence, the girl walked into her playhouse (Figure 2A) and pulled the door shut behind her. Once inside she explored the interior, stopping in front of the dish sink to perform a bouncy, dancy motion before going to the window and leaning out. She appeared to make eye contact with the videographer and made a face that she sometimes used to express surprise and other times to flirt with (Figure 2B). Then she executed a Queen Elizabeth-style wave, moving her fingers to her palm while holding her arm still (Figure 2C). Returning to the front door, she stood in the doorway 
and again made her special face (Figure 2D) before finally carrying out a chair, placing it front of the doorway and sitting down as if to preside over her home (Figure 2E).

The child in this video already had specific body habits that we (Euro-American) adults associate with femininity. She waved discretely with her hand rather than using a larger arm movement. She engaged socially with the videographer, and flirted using exaggerated facial movements. By 15 months embodied gender/ sex is becoming visible to adult observers. Can we find earlier manifestations or dyadic behaviors that lead to this moment? So far in this essay, we have offered a framework, supported and shaped by existing literature to link the registering of sensory input with a child's nervous and motor systems on the one hand, and systems of gender/sex on the other. In the empirical portion of our work we asked whether we could, during months three to 12 , observe behaviors that differed in frequency or duration in motherson compared to mother-daughter dyads as they played together. Our findings may help to design better studies, using more fully-formed hypotheses that can illuminate the interlinked, hierarchically-connected processes through which gender/sex identities emerge, are sustained, and modulate -in this case in infancy and toddlerhood-but more generally throughout the life cycle.

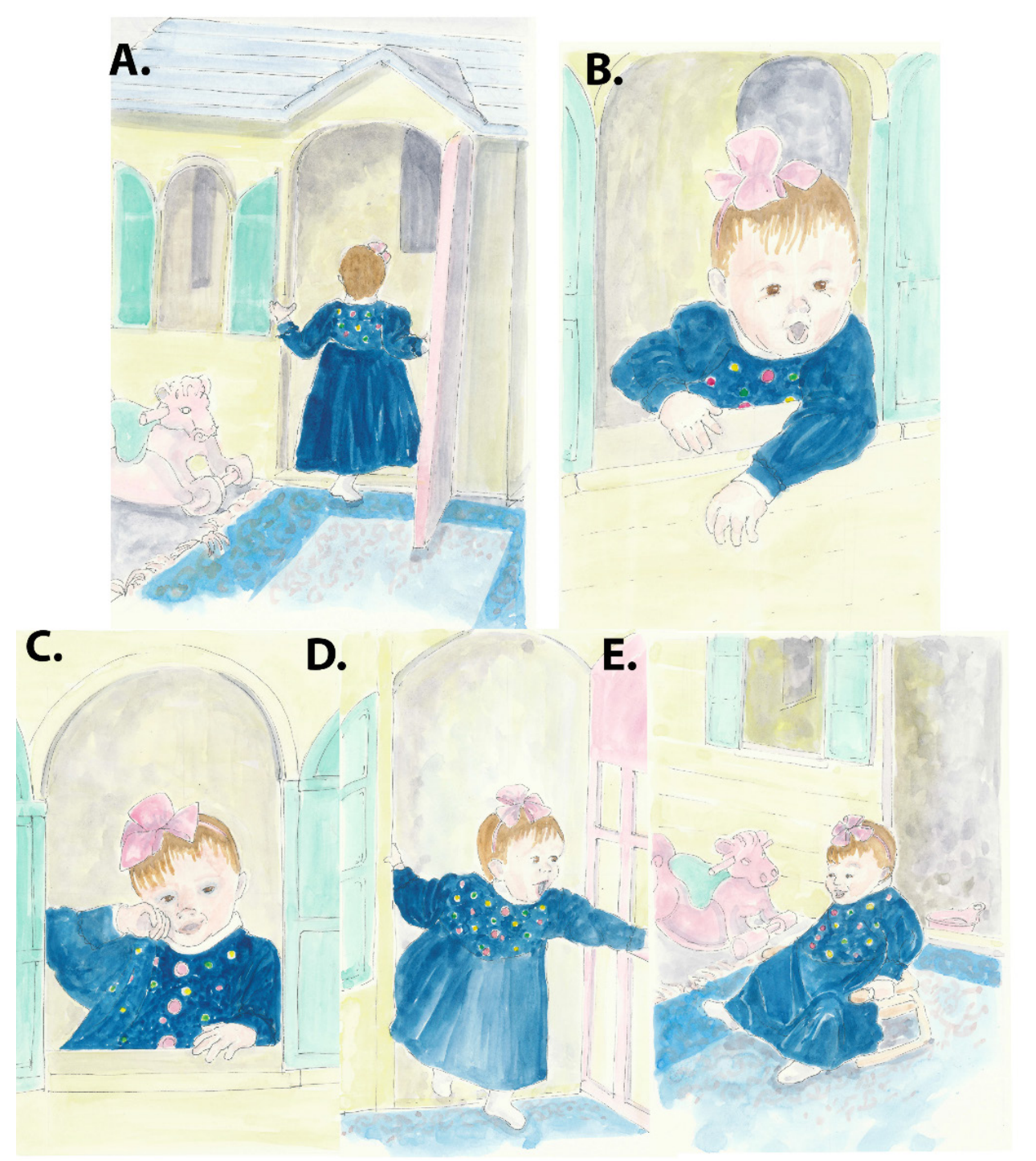

Figure 2: Playhouse sequence showing gender/sex embodiment in a 15-month-old girl. See text for extended description. Drawings by Thea Ernest. (c) first author. 


\section{Methods}

\section{Participants, Taping, and Initial Data Collection}

The observations for this study come from videotapes originally recorded during 1989 and 1990 for a study of infant temperament (Seifer, Sameroff, Barrett, \& Krafchuk, 1994). From a set of 50 infants (24 birth-identified boys and 26 birth-identified girls), we selected those families for whom we had the most complete sets of data, i.e., the ones that started as early as three months and went past twelve months. Then, by matching girls and boys, to the extent possible, by weekly age at the time of each observation, we chose a subset of 30 families, 15 with boys and 15 with girls. The participants were first-time mothers and their fullterm babies. None of the mothers worked full time during their child's first year (Vaughn, Contreras, \& Seifer, 1994); Seifer et al., 1994). The families were middle and working class. The Hollingshead four-factor SES averaged 1.96 (SD = .75 , range 1-4). The mothers' average age at the child's birth was 29.1 years ( $S D=4.2$ years, range 22.2-36.9 years).

The original videotapes, made during hour-long home visits, consisted of three sessions-the infant playing alone, the infant and mother playing together (our coding target), and one or more caretaking episodes such as diapering, feeding or bathing the infant. Before beginning detailed coding, we identified the mother-infant play session. To ensure a full five-minute period, coding began at two minutes after the start of the joint play session. For example, if the scene switched to mother and infant playing together at 12 minutes and 24 seconds into a videotape, the observation would begin at 14 minutes and 24 seconds.

With two mother-infant play observations per month for each of the nine months, we ideally had 18 observations per infant. In practice, there were missing observations. Two infants (one male and one female) were missing three observations, eight (four males and four females) were missing two observations, seven (five males and two females) were missing one observation each. The remaining thirteen infants (five males and eight females) had all eighteen observations. Statistically, we mitigated the effects of these missing points by combining monthly data into twomonth groups.

Infant and maternal behaviors and play type were coded on a second-by-second basis using Observer XT 7 software designed by Noldus Information Technology (https://www. noldus.com/the-observer-xt/gathering-data). This software permits all coding to be entered on parallel timelines that can be visualized in the moment or exported for analysis with SPSS. Details on the organization of coding teams can be found in Appendix 1.

\section{Code Descriptions}

\section{Coding Manual}

Our complete coding manual may be found in Appendix 1. Below we offer descriptions of the codes focused on in this essay. The results of other codes may be found in previous publications (Sung, Fausto-Sterling, García Coll \& Seifer, 2013; Fausto-Sterling, Crews, Sung, García Coll \& Seifer, 2015).

\section{Dyadic Play Type}

In Table 2 we describe five coded mother-infant (dyadic) play types. These consist of sequences of communicative co-action, defined and analyzed as a dynamically stable relational pattern (Fogel, Garvey, Hsu, \& West-Stroming, 2006). Fogel and colleagues referred to the same list of five codes as frames while Bakeman and Adamson (1984) called them types of joint engagement. Coders specified mother-infant play types, by distinguishing segments of communication based on the direction of the mother's and infant's attention, the mother's and the infant's location, the mother's and the infant's' postural co-orientation, and the topic of their communication.

\section{Maternal Behaviors}

We define the codes describing maternal behaviors that were sufficiently frequent to permit statistical analysis in Table 3. These involve the mother touching the infant in order to facilitate infant motor activity including repositioning or moving the infant from one spot to another.

\section{Maternally Supported Infant Standing}

As we examined our initial results for maternally supported infant standing, we began to suspect the existence of more nuanced differences in the ways mothers of boys and mothers of girls supported infant standing. Based on these observations, we developed two subsidiary hypotheses: (1) during assisted standing, mothers held daughters closer to their bodies than they did sons and (2) mothers' speech content during assisted standing differed between mother-son and mother-daughter dyads. Specifically, we hypothesized that mothers of sons were more verbally encouraging of movement and more likely to praise their sons' strength and size. To test these propositions, we revisited previously coded instances of maternally supported infant standing and applied a new code for body distance, 
Table 2

Descriptions of Mother-Infant Play Type Codes

\begin{tabular}{ll}
\hline \multicolumn{1}{c}{ Code } & \multicolumn{1}{c}{ Definition } \\
\hline Joint object play & $\begin{array}{l}\text { Mother and infant play jointly with an object. Mother takes } \\
\text { active role demonstrating, scaffolding infant's use of object. } \\
\text { Mother and infant using same toy simultaneously or tossing toy } \\
\text { back and forth. Some authors refer to this as guided object play }\end{array}$ \\
Motor-Social play & $\begin{array}{l}\text { Mother plays using gestures alone (no objects). Examples } \\
\text { include physical motor play, patty cake, and maternal gestures } \\
\text { that encourage infant motor movement. }\end{array}$ \\
Child-directed play & $\begin{array}{l}\text { Infant plays with objects without mother's direct assistance but } \\
\text { with her ongoing visual attention and/or postural support. Only } \\
\text { the infant (not the mother) holds the object. Some authors refer } \\
\text { to this as not-guided or passive object play. }\end{array}$ \\
Physical-joint object play & $\begin{array}{l}\text { Elements of simultaneous face-to-face motor social and guided } \\
\text { object play as when mother and infant interact with each other } \\
\text { whixed }\end{array}$
\end{tabular}

Table 3

Maternal Behavior Code Descriptions

\begin{tabular}{|c|c|c|}
\hline Code & Subcode & Definition \\
\hline Gross motor stimulation & & $\begin{array}{l}\text { Mother moves infant arms or } \\
\text { legs to mimic infant behaviors } \\
\text { such as kicking or flailing }\end{array}$ \\
\hline \multirow[t]{3}{*}{ Assisted locomotion: } & & $\begin{array}{l}\text { Mother holds infant to facilitate } \\
\text { standing or walking }\end{array}$ \\
\hline & Holding-large limbs & $\begin{array}{l}\text { Mother holds infant's arms, } \\
\text { torso or legs as part of assisting } \\
\text { movement }\end{array}$ \\
\hline & Shift infant & $\begin{array}{l}\text { Mother repositions infant with } \\
\text { little or no impetus from infant }\end{array}$ \\
\hline Lift up & & $\begin{array}{l}\text { Mother lifts infant as a form of } \\
\text { motor play; does not include } \\
\text { repositioning }\end{array}$ \\
\hline \multicolumn{3}{|l|}{ Affectionate touch } \\
\hline & & $\begin{array}{l}\text { Refers to any sort of touch or } \\
\text { behavior that primarily conveyed } \\
\text { affection, e.g. tickles, nuzzles, } \\
\text { kisses, stroking and hugging }\end{array}$ \\
\hline Maternal Vocalization & & $\begin{array}{l}\text { Refers to all vocalizations, } \\
\text { including speech, babble to } \\
\text { infant and other noises }\end{array}$ \\
\hline
\end{tabular}


which we recorded as maternal "arm distance". We used scores of 1-4: (1) mother holds baby with outstretched arms (Figure 3A), (2) mother holds baby with upper and lower arms bent at the elbow at 90 degrees (Figure 3B), (3) mother holds baby with upper and lower arms bent at the elbow at 45 degrees (Figure 3C), and (4) mother holds baby close to body (Figure 3D). Therefore, lower average body-distance scores indicated a baby's more physically distant interaction with the mother. We also measured the presence or absence of maternal vocalization during standing events. If vocalization occurred, we transcribed the complete content and assigned it to one of five categories: (1) general, (2) babbling, (3) encouraging movement, (4) general praise, and (5) praise about body size or strength. Thus, when averaging these scores, higher average numbers indicated infants who received more general praise and praise about body size and strength. For this specific study, we obtained an average kappa of .73.

\section{Infant Behaviors}

Brief descriptions of the infant activity codes for which we present results are listed in Table 4. Fuller descriptions may be found in the Coding Manual in Appendix 1.

\section{Rater Reliability}

Before coding the specific behaviors described above, coders identified the play types (Table 2 ) in which the dyad engaged throughout the five-minute sequence. Then they coded maternal-specific or infant-specific behaviors within each play episode. During their initial training, coders were told that the study's purpose was to collect longitudinal data on mother-infant interaction. We assigned specific behavioral code sets to each coding team, discussing with them the operational definitions of the variables and sub-variables they were to code. To score as an agreement or disagreement, we used the Observer XT 7 reliability calculator (Jansen, Wiertz, Meyer, \& Noldus, 2003) and set
Figure 3: Examples of body distance. A. Outstretched B. Mother's arms at $90^{\circ}$ angle $\mathrm{C}$. Mother's arms at $45^{\circ}$ angle $\mathrm{D}$. Mother holds baby close to the body. Drawings by Thea Ernest. (c) first author.
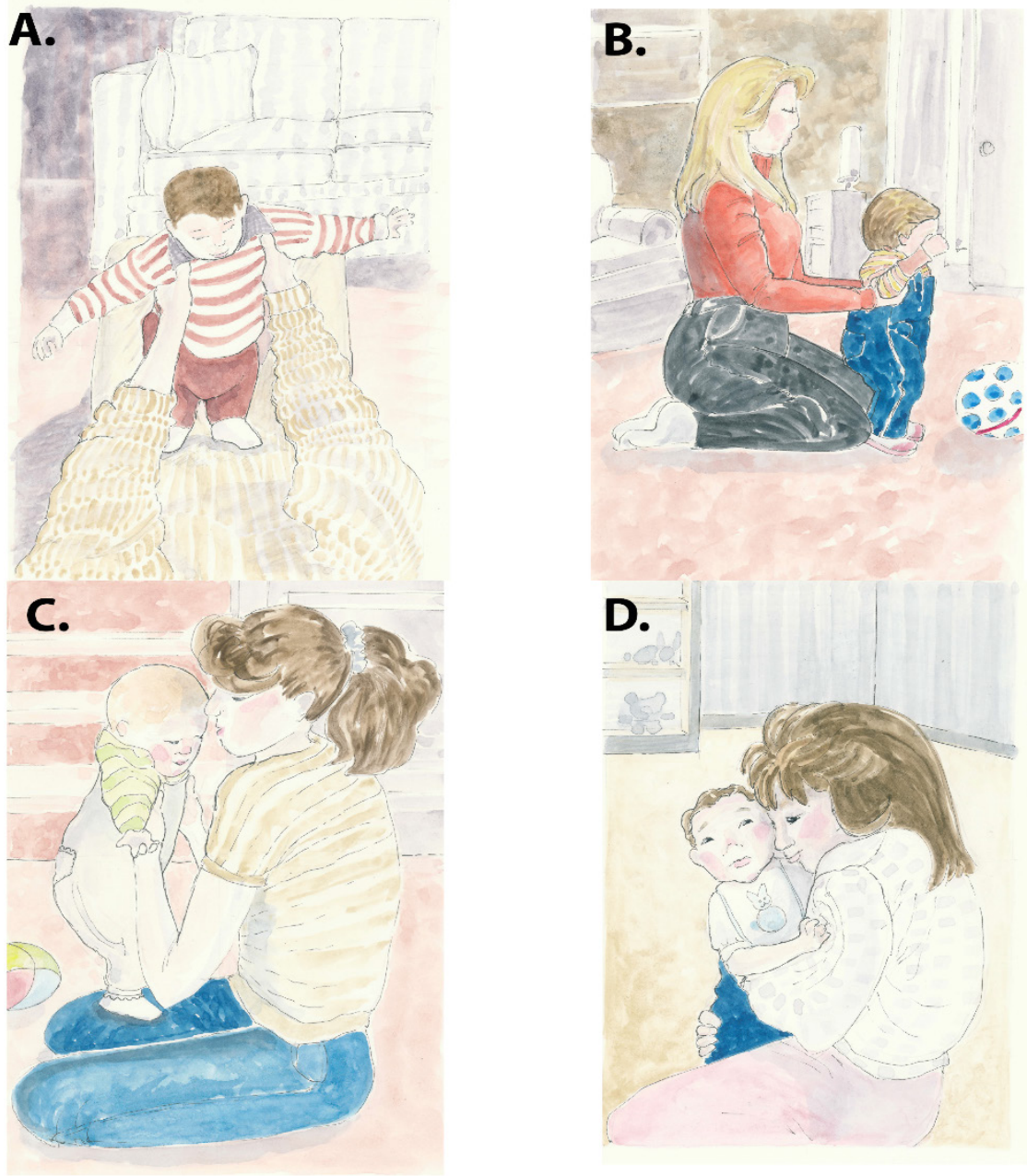
Table 4

Descriptions of Infant Motor Behavior Codes

\begin{tabular}{|c|c|c|}
\hline Code & Subcode & Definition \\
\hline \multirow[t]{4}{*}{ Lying: } & & Infant lies horizontally on front, back or side \\
\hline & Still & Infant does not independently change position \\
\hline & Roll over & Infant rolls over at least 90 degrees \\
\hline & Move or kick & Any gross motor movement of legs \\
\hline \multirow[t]{5}{*}{ Sitting: } & & $\begin{array}{l}\text { Infant sits upright, not strapped to parent or supported by } \\
\text { hands }\end{array}$ \\
\hline & Independent & Infant sits without additional support \\
\hline & Mother-assisted & Infant sits with support from mother \\
\hline & Object-assisted & Infant sits in a seat with back support \\
\hline & Rock-Lean-Bounce & Infant rocks or bounces on its own while sitting \\
\hline Crawling & & $\begin{array}{l}\text { Infant moves by some means other than walking, } \\
\text { including scooting; also stationary crawling position }\end{array}$ \\
\hline \multirow[t]{5}{*}{ Standing: } & & Infant upright on feet without walking \\
\hline & Independent & Infant does not hold on to mother or any object \\
\hline & Mother-assisted & $\begin{array}{l}\text { Mother assists standing by supporting infant trunk or } \\
\text { hands }\end{array}$ \\
\hline & Object-assisted & $\begin{array}{l}\text { Infant uses furniture or a walker to stand with both feet } \\
\text { on ground and weight on the legs and feet }\end{array}$ \\
\hline & Rock-Lean-Bounce & Infant rocks or bounces on its own while standing \\
\hline
\end{tabular}

the tolerance window at two seconds.

Initially, two research assistants double-coded about $15 \%$ of the approximately 505 observations of motherinfant play together scenes. Throughout the long period of data collection, we instituted a regular sequence of spot-checking Kappas via double coding. The inter-rater reliabilities for the codes used in this paper are listed in Appendix 1. All of the codes for which we here present data had a kappa greater than or equal to .70 .

\section{Analyses and Graphing}

\section{Longitudinal Analysis}

Our research aim for this segment of our analysis was to compare developmental patterns of coded behaviors for mother-son and mother-daughter dyads from three to 12 months, and to display the results in a visually accessible manner. We wanted to learn more about individual dyad variation as well as detect the possible emergence of group differences related to the infant's gender/sex. To visualize individual dyadic developmental patterns, we used the steps described by Singer and Willet (2003). We first plotted age at observation (in weeks) on the $\mathrm{x}$-axis and frequency or duration of coded events on the $y$-axis for individual dyads, mothers or infants. Next, using ordinary least squares (OLS) regressions, we transformed these into smooth curves for each dyad, mother or infant. After running a withindyad or within-person linear change regression model separately for each dyad or dyad member, we plotted all individuals or dyads on a single graph (spaghetti plot) thus allowing inter-family comparisons. Before taking the previous step, we grouped the results into two-month bins to allow a smoother visual presentation, or as Singer and Willet wrote "to decrease clutter" (p. 54). On the combined graphs we also plotted an average change trajectory for the entire group. We used the graphing and linear regression programs in the software program GraphPad Prism (https:/l www.graphpad.com/guides/prism/7/curve-fitting/index. 
htm?reg analysischeck linearreg.htm). Finally, also using GraphPad Prism, we used the $\mathrm{F}$ test of overall significance in linear regression to test for significant differences between male and female infants or their mothers in either slopes or $\mathrm{Y}$ intercepts and to produce a unified graph of the data from dyads, or from boys, girls, or their mothers. Within GraphPad, if the slopes differ significantly, it is not possible to compare $Y$ intercepts.

\section{Three-dimensional Scatterplots, Mesh Plots and K-means Cluster Analysis}

Because we hypothesize that the presymbolic gender/sex development results from the repetition of complex, multimodal sensory chunks (see previous section on statistical learning and informational chunks), we wanted to explore methods that allow us to visualize and compare multidimensional, multimodal interactions. We examined the composite (i.e. including all subcodes) assisted locomotion code in order to map how it co-existed with maternal vocalization and affectionate touch. To this end, we compared mother-son and mother-daughter dyads from three to 12 months of age using the SigmaPlot (https:// systatsoftware.com/products/sigmaplot/) graphing program. We first made three-dimensional (3D) scatterplots with maternally assisted locomotion represented by distance along the $x$-axis, maternal vocalization as distance along the $y$-axis, and affectionate touch plotted along the z-axis.

To draw the mesh plots (sometimes called wireframe plots) SigmaPlot fits a smooth surface to the XYZ data used for the 3D scatterplots. Because it constructs the $z$-axis as a function of the $X$ and $Y$ data, the resulting surface does not necessarily go through each point. Instead, the drawn surface relies on estimating values in places where there are no data. Since SigmaPlot does not reveal the exact functions used to draw the surfaces, we cannot use the mesh plots, as we do the scatter plots, for precise quantitative estimates. Instead, we see the mesh plots as a tool to visualize, during similar developmental time periods, whether populations of mother-son and mother-daughter dyads occupy different 3D state spaces for the three plotted behaviors.

Visual inspection of the scatter and mesh plots suggested that, for certain time periods, mother-son and motherdaughter dyads occupied different (although overlapping) 3D state spaces. To explore this impression, we used the K-means cluster tool in SPSS. First, we examined the distribution of behavioral combinations for possible clusters. Then we tried to determine whether cluster membership differed by sex of the infant. To do this we ran a K-means cluster analysis on the full data set (mother-son and mother-daughter dyads combined). We set the tool to provide three clusters and used the ANOVA function to indicate which behaviors might contribute significantly to the clustering. The K-means cluster analysis generated a new variable that indicated the final cluster membership (Cluster 1, 2 or 3) of each dyad. We next used the cross-tabulation procedure on this new variable to map which cluster number each individual dyad belonged to against sex of infant. We displayed the results as bar graphs. We were unable to use a Chi-Square test to assess the null hypothesis that the distribution of cases within each cluster is the same across categories of sex of infant. This inability resulted from the small size of our starting sample, subdivided by assigning our sample into one of three clusters, and then further subdivided by the cross-tabulation by sex of infant within each cluster.

\section{State Space Grids}

We made use of state/space grids in our analysis of gender/sex differences in the lie/still code. To do so, we used the Noldus Observer XT software to transform our original second by second data into trajectories suitable for Gridware analysis (Gridware Version 1.15a, Gridware, Oct. 27, 2017). For the current analysis we were particularly interested in calculating transitional propensity and transitional entropy (Dishion, Bullock, \& Granic, 2002; Hollenstein, 2007, 2013). Gridware defines transitional propensity (TP) as the number of transitions from origin to destination divided by the duration in origin divided by the transitional bin size (the shortest time of a dyadic event during the time period observed). TP is a conditional probability that a mother-infant dyad in a particular twodimensional state space will move in a set time period to a certain destination state space. Entropy is a measure of organization or predictability: low entropy suggests a highly organized and predictable pattern and high entropy indicates a less predictable behavior set. Gridware defines transitional entropy as TP multiplied by $\ln (1 / T P)$.

\section{Study Family Anonymity}

For two of our figures, we began by extracting still shots from our videos. In order to preserve anonymity, however, we hired an artist to draw watercolor figures that are based on the original stills. The first author worked closely with the artist to maintain accuracy on key points (e.g. arm angles or a particular facial expression) while changing identifying details such as clothing or hairstyle. 


\section{Results}

\section{Large Gender/sex Differences}

von Eye, Bergmann \& Hsieh (2015) presented the principles of what they call "person-oriented research", which draws "conclusions in terms of the patterns that describe individuals or groups of individuals" (p. 8) The approach to longitudinal analysis described in the methods section allows us to see the range of individual differences while also discerning significant group differences in behavior patterns over time. We ran longitudinal analyses for both duration and frequency for all of the play types, maternal behaviors, and infant behaviors described in the Methods section and in the coding manual (Appendix 1), that is, upwards of 50 comparisons. Most of these showed no significant differences (with the standard $p \leq .05$ measure) in developmental pattern (slope) or elevation (y-intercept). Because of concerns about the large number of comparisons made, in this report we heightened our criterion for significance, by emphasizing findings for which $p<.01$.

\section{Play Type}

Table 5 shows the results of longitudinal analyses for frequencies and duration of motor-social play type, two maternal behaviors (assisted shift and lifting up) and two infant behaviors (lie:still and sit:assisted mother). The developmental patterns for mother-daughter and motherson dyads were similar (same slope) for motor-social play frequency (Table 5). However, comparison of the $y$-intercepts on these parallel lines shows that the duration of motor-social play was significantly longer for mother-son dyads throughout the observed developmental period, $F(1,147)=12.79, p=$ .0005 (Table 5; Figure 4). For both dyads with boys and with girls the duration of motor-social play is highest in the first few months and declines with increasing infant age.

Figure 4 shows the pattern for duration of motorsocial play over time for each dyad. Visual inspection of the clustering of dyads around the regression line reveals that in Figure 4A (mother-daughter dyads) there are four families with a pattern of well under 50 seconds per month group of motor-social play. In contrast, there are no such families (Figure 4B) for mother-son dyads. This means that although most mothers engage in motor-social play with their sons and daughters for similar durations and that the change with time (same slope) is also the same, four of the 15 mother-daughter dyads show a different pattern. For these dyads there is very little motor-social play throughout the study period.

\section{Maternal Motor Behaviors}

Mothers frequently moved their babies from one position to another. Here too, the slopes for both frequency $F(1,146)=$ $2.0, p=.16$ and duration $F(1,146)=0.05, p=.82$ of assistshift were the same for mothers of both sons and daughters. In both cases, however, the elevations ( $y$-intercept) differed significantly (Figure 5). The y-intercept for assist-shift frequency for mothers of boys was 1.6 times higher than for mothers of girls $F(1,147)=20.07, p<.0001$. The $Y$ intercept for assist-shift duration was 1.9 times higher $F(1$, $147)=34.11, p<.0001$. Figure 5 displays individual dyad patterns for duration of the assist/shift behavior, as well as a summary graph of the analysis.

One other maternal behavior, the frequency of lift up (Figure 6), showed large differences for mothers of girls compared to mothers of boys. We applied this code to instances when the mother plays with the infant by lifting it into the air. While all of the other observed differences reported in Table 5 had the same developmental pattern (same slope) but different frequencies or durations, the frequency of the lift up behavior over our 9-month study period had a different slope for mothers of sons compared to mothers of daughters $F(1,146)=10.37, p<.002$. As can be seen in Figure 6, about half of the boys' mothers frequently lifted them up during months three to six. Later the frequency of this behavior leveled off for all dyads. The duration of lift up behaviors were also longer for mothers of boys, but the level of significance is less striking $F(1,146)=$ $5.66, p<.02$.

\section{Infant Motor Behaviors}

Table 5 shows two robust male-female differences in infant motor behaviors. The first is for the $y$-intercept of lines for the lie:still code (Figure 7). The slopes for both male and female lines did not differ $F(1,146)=0.90, p=.34$; however the $y$-intercept, or line elevation, was almost 1.4 times higher for the boys than the girls $F(1,147)=7.29, p=.008$. As shown in Figure 7, there were no obvious sub-patterns or clusters for either the boys or the girls.

Given the many reports of higher activity levels for boys compared to girls (Campbell \& Eaton, 1999), we were surprised to observe in our sample that on average boys lay still more often than girls. Since we found that mothers stimulated or moved sons more often or for longer duration than for daughters, we wondered whether moments of inactivity induced mothers to pick up and physically stimulate their children. To gain insight into dyadic dynamics during periods of still lying, we used Gridware (Hollenstein, 2007; 2013) to examine maternal responses 
Table 5

Slopes and y-intercepts of selected dyad, infant and maternal behaviors

\begin{tabular}{|c|c|c|c|c|c|c|}
\hline \multirow{2}{*}{ Behavior } & \multicolumn{2}{|c|}{$y$-Intercept } & \multirow{2}{*}{ Significance } & \multicolumn{2}{|c|}{ Slope } & \multirow{2}{*}{ Significance } \\
\hline & Boys & Girls & & Boys & Girls & \\
\hline \multicolumn{7}{|l|}{$\begin{array}{l}\text { Motor-social play } \\
\text { type }\end{array}$} \\
\hline Frequency & 2.14 & 1.77 & $\mathrm{~F}(1,147)=1.51, p=.22$ & -0.10 & -0.08 & $F(1,146)=0.19, p=.66$ \\
\hline Duration & 165.40 & 122.70 & $F(1,147)=12.79, p=.0005$ & -11.96 & -9.74 & $F(1,146)=0.68, p=.41$ \\
\hline \multicolumn{7}{|c|}{ Assist shift (Mother) } \\
\hline Frequency & 6.91 & 4.40 & $F(1,147)=20.07, p<.0001$ & -0.43 & -0.29 & $F(1.146)=1.58, p=.21$ \\
\hline Duration & 26.95 & 13.99 & $F(1,147)=34.11, p<.0001$ & -1.51 & -0.88 & $\mathrm{~F}(1,146)=1.49, p=.22$ \\
\hline \multicolumn{7}{|l|}{ Lift up } \\
\hline Frequency & 1.76 & 0.45 & Can't test & -0.16 & -0.02 & $F(1,146)=10.37, p<.002$ \\
\hline Duration & 9.37 & 2.94 & Can't test & -0.85 & -0.15 & $F(1,146)=5.66, p<.02$ \\
\hline \multicolumn{7}{|l|}{ Lie:Still (Infant) } \\
\hline Frequency & 3.55 & 2.55 & $F(1,147)=7.29, p=.008$ & -0.28 & -0.21 & $F(1,146)=0.90, p=.34$ \\
\hline Duration & 153.90 & 161.70 & $F(1,147)=.02, p=.90$ & -14.84 & -16.12 & $F(1,146)=0.15, p=.70$ \\
\hline \multicolumn{7}{|c|}{ Sit:Assisted mother (Infant) } \\
\hline Frequency & 2.91 & 1.65 & $F(1,147)=16.48, p=<.0001$ & -0.17 & -0.09 & $F(1,146)=2.0, p=.16$ \\
\hline Duration & 101.80 & 90.52 & $F(1.147)=.71, p=.40$ & -6.49 & -5.84 & $F(1,146)=0.05, p=.82$ \\
\hline
\end{tabular}

to male versus female infants as they lay still. We analyzed times during which differences in the still lying behaviors or the hypothesized maternal response were most evident and specifically measured the transitional propensity (TP) and transitional entropy (TE) of moving from a still lying state to each of the following maternal behaviors: stimulate gross motor activity, lift up, assist shift, and holding. After importing measures from Gridware into SPSS we aggregated the data by family and dyad composition before performing a simple ANOVA. The TP from an origin of still lying still to a destination of maternal shift did not differ by infant gender/sex during months three to 12 . However, there was a significant effect on Transitional Entropy, $F(1,28)=7.226$, $p=.012$, Cohen's $d=.988, r=.44$, with mother-son dyads exhibiting greater transitional entropy than mother-daughter dyads. The TP from an origin of still lying to a destination of stimulate gross motor also did not differ by infant gender/ sex during months three to six. However, there was a significant gender/sex-of-infant effect on Transitional Entropy, $F(1,28)=4.413, p=.045$, Cohen's $d=.767, r=$ .358 , with mother-son dyads exhibiting greater transitional entropy than mother-daughter dyads. Finally, neither the TP nor TE for the origin lie:still to the destination of hold using gross motor muscles differed significantly by gender/sex of infant and there were too few cases of still lying or lift up to do a statistical analysis.

The second large difference for infant behaviors was for the code sit:assisted mother. The slopes for both the frequency and duration of this behavior did not differ (for frequency: $F(1,146)=2.0, p=.16$ and duration: $F(1,146)$ $=0.05, p=.82)$. The elevation (y-intercept) of the boys' regression line for sit:assisted mother frequency, however, was 1.76 times higher than the girls' $(F(1,147)=16.48, p=$ $<.0001)$. Figure 8 displays the frequency of individual dyad patterns as well as a summary graph of the analysis. One impression taken from this figure concerns the clustering of individual mother-daughter dyad lines around the mean regression line. This compares to a wider variability for the mother-son dyads. For example at 3-4 months the range among different mother-daughter dyads for sit:assisted mother frequency was zero to two, while for their motherson counterparts the range was from 1.5 to 5.25 (data taken from raw tables used to make spaghetti plots). 
Figure 4: Motor-social Playframe
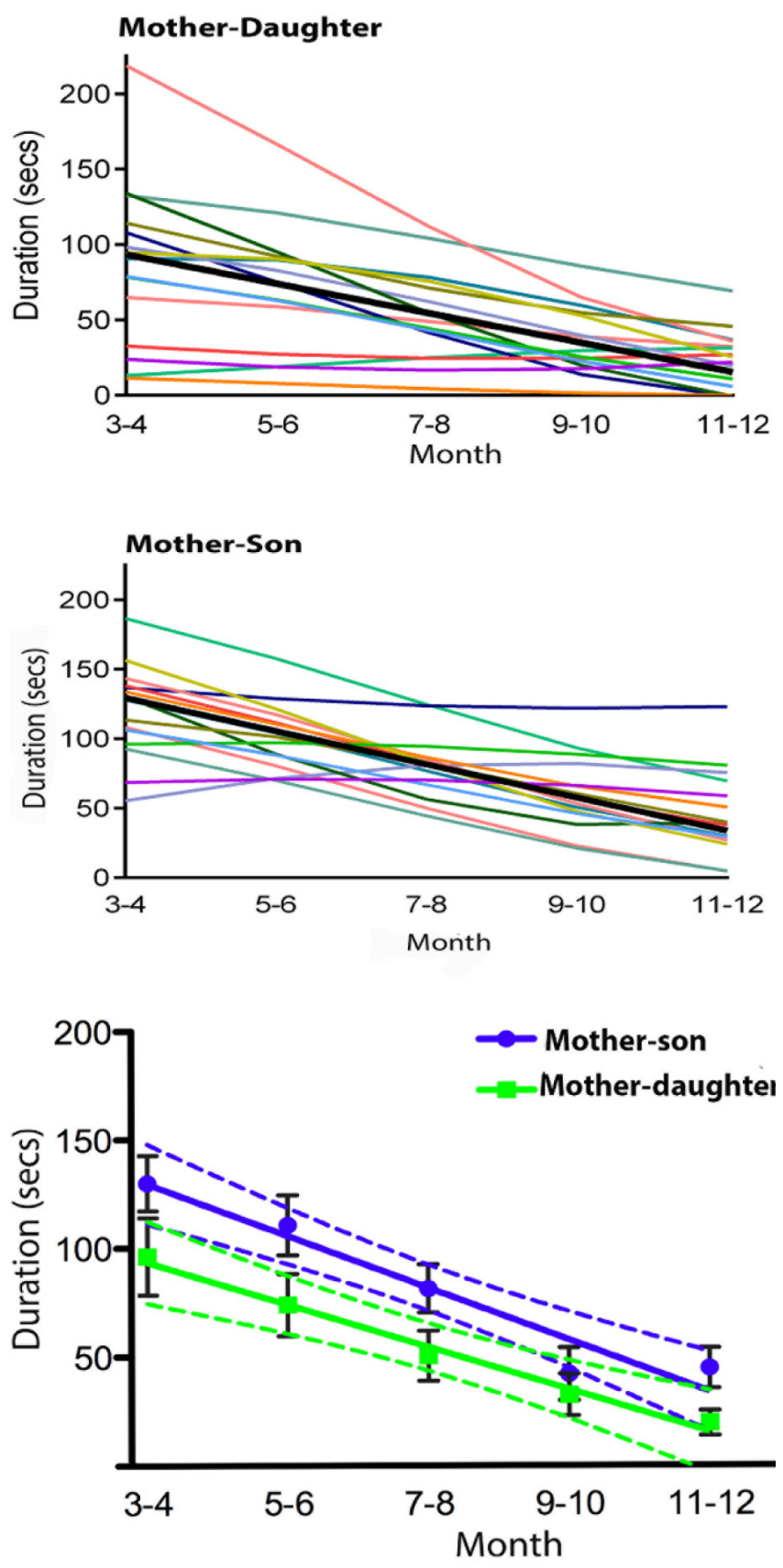

Figure 4: Motor-Social Play Type. Duration in 2-Month groups from 3-12 months. Top panel shows individual dyads with girls (thin colored lines) and a regression line for dyads with girls (thicker black line). Middle panel shows individual dyads with boys (thin colored lines) and a regression line for dyads with boys (thicker black line). The bottom panel shows group regression lines and standard deviations. (Square symbol, green line = mother-daughter dyads; round symbolblue line $=$ mother-son dyads).
Figure 5: Assist Shift
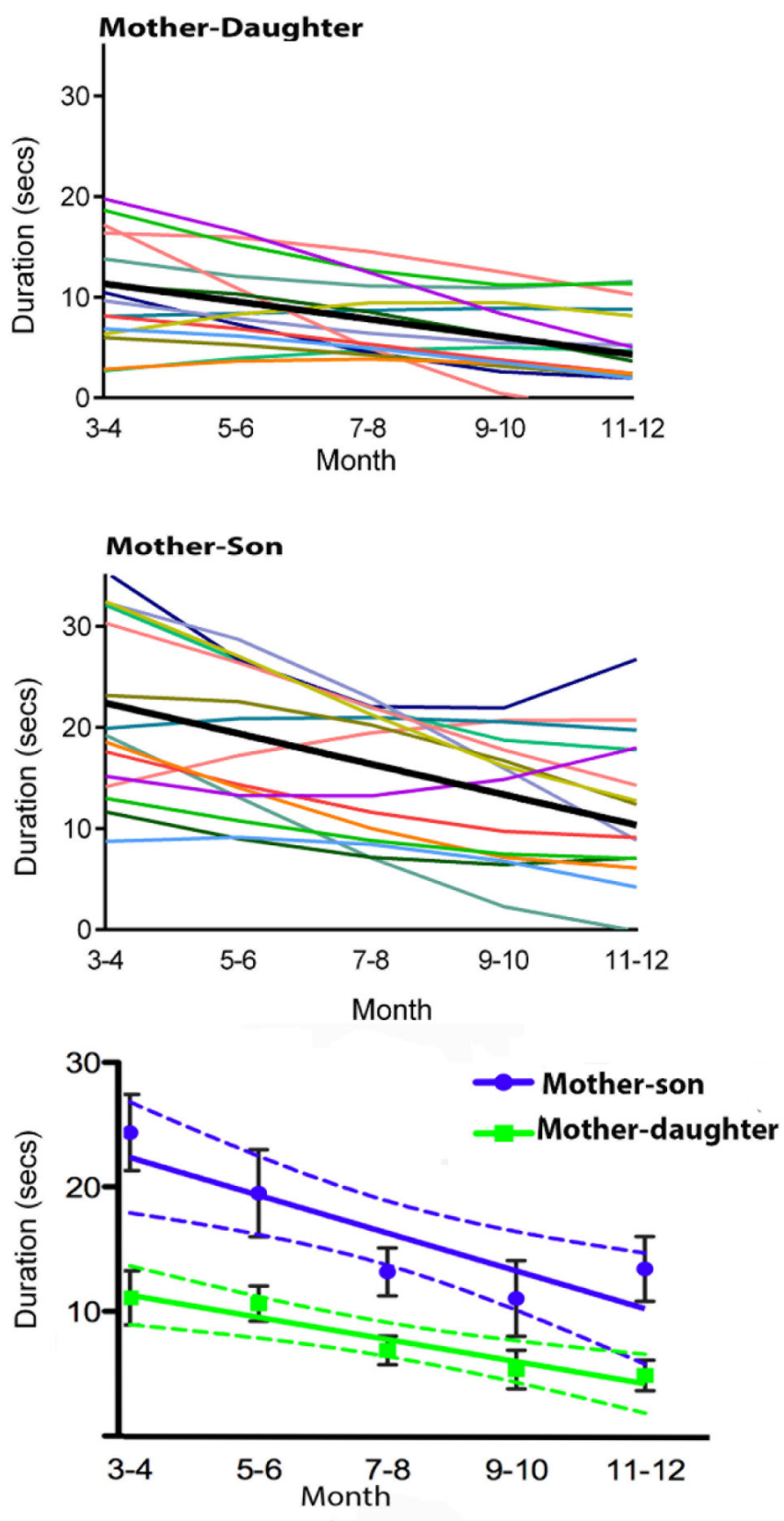

Figure 5: Assist Shift. Duration in 2-Month groups from 3-12 months. Top panel shows individual dyads with girls (thin colored lines) and a regression line for dyads with girls (thicker black line). Middle panel shows individual dyads with boys (thin colored lines) and a regression line for dyads with boys (thicker black line). The bottom panel shows group regression lines and standard deviations. (Square symbol, green line $=$ mother-daughter dyads; round symbol-blue line $=$ mother-son dyads). 
Figure 6: Lift Up
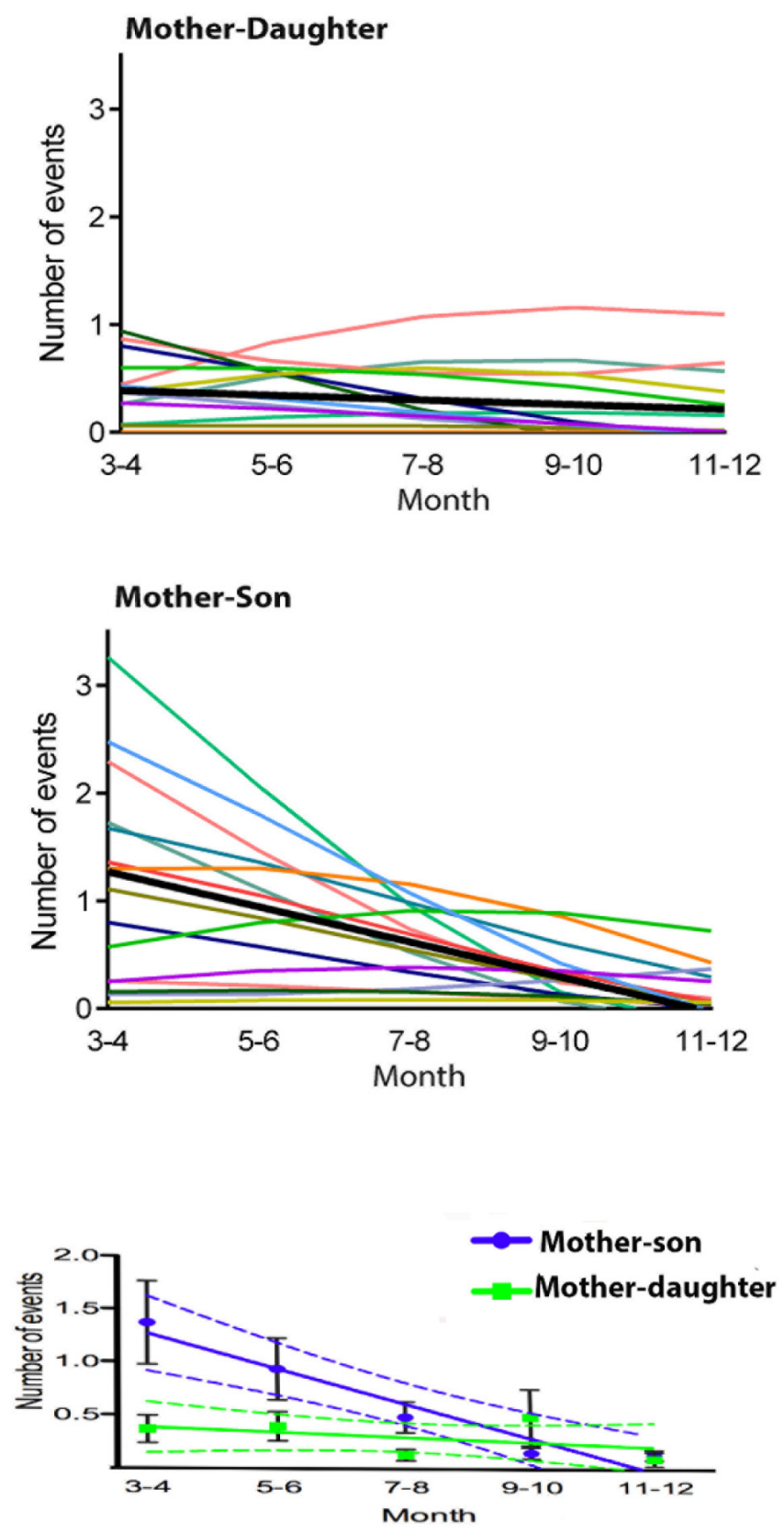

Figure 6: Lift Up. Frequency in 2-Month groups from 3-12 months. Top panel shows individual dyads with girls (thin colored lines) and a regression line for dyads with girls (thicker black line). Middle panel shows individual dyads with boys (thin colored lines) and a regression line for dyads with boys (thicker black line). The bottom panel shows group regression lines and standard deviations. (Square symbol, green line $=$ mother-daughter dyads; round symbol-blue line = mother-son dyads).
Figure 7: Lie-still
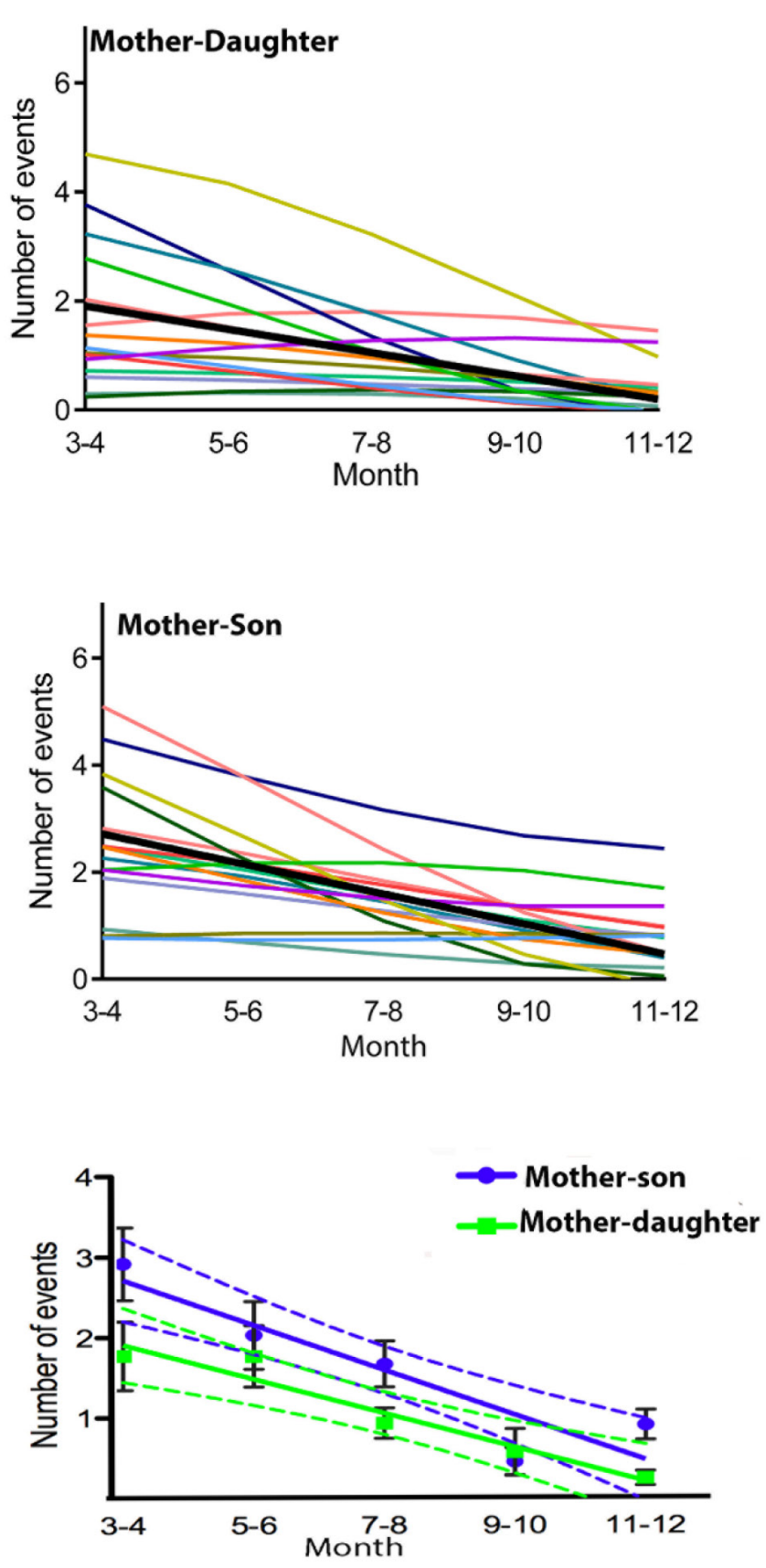

Figure 7: Lie:still. Frequency in 2-Month groups from 3-12 months. Top panel shows individual dyads with girls (thin colored lines) and a regression line for dyads with girls (thicker black line). Middle panel shows individual dyads with boys (thin colored lines) and a regression line for dyads with boys (thicker black line). The bottom panel shows group regression lines and standard deviations. (Square symbol, green line $=$ mother-daughter dyads; round symbol-blue line $=$ mother-son dyads). 


\section{Talking and Touching while Assisting Locomotion}

Three-dimensional state space comparison of mother-son and mother-daughter dyads

Above, we reported findings of significant gender/sexrelated differences in individual maternal behaviors. In this section we examine a more complex situation. Ferber, Feldman and Makhoul (2008) reported that during the first year of development, affectionate touch played an important role in dyadic synchrony for both mother-daughter and mother-son dyads. We built on these findings by studying gender/sex differences in positive, stimulatory and instrumental maternal touch in mother-son and mother daughter dyads during the first year of life (Fausto-Sterling, Crews, Sung et al, 2015). We reported significant gender/sex $x$ age interactions for both instrumental (assists infant in moving, sitting, or handling an object) and stimulatory (play lifting, gross motor stimulation such as"bicycle" play, and rocking and jiggling the infant) touch but no significant gender/sex $x$ age interactions for positive touch (affectionate touch and caretaking such as adjusting appearance). Our group also reported that mothers vocalized more to daughters than sons during the first several months of infancy (Sung, Fausto-Sterling, García Coll, \& Seifer 2013). In this segment of our essay we ask what happens when we look at these behaviors-two of which (assisted locomotion and maternal vocalization) show gender/sex differences in maternal behavior and one of which seems not to-as an interactive group? Are the differences synergistic? Do differences in affectionate touch, which are not evident overall, appear within specific behavioral suites? We explored these possibilities by examining three-dimensional state spaces for assisted locomotion, maternal vocalization and maternal affectionate touch.

As with the spaghetti graphs, we visualized our results using graphs that displayed individual families as well as aggregate results of three behaviors-- assisted locomotion (using the master code that includes the sub codes examined in the previous section), maternal vocalization (including speech and other meaningful sounds as well as babbling) and offering affectionate touch. For the frequency of assisted locomotion the slopes for mother-daughter $(-0.73)$ compared with mother-son dyads $(-1.45)$ differed with marginal significance $F(1,146)=5.4, p=.02$. For the duration of assist locomotion the slopes for mother-daughter (-4.60) compared with mother-son (-7.05) dyads did not differ $F(1,146)=1.81, p=.18$. The $y$-intercepts (sons = 98.56 , daughters $=67.73$ ), however, differed significantly $F$

\section{Figure 8: Sit-assisted Mother}
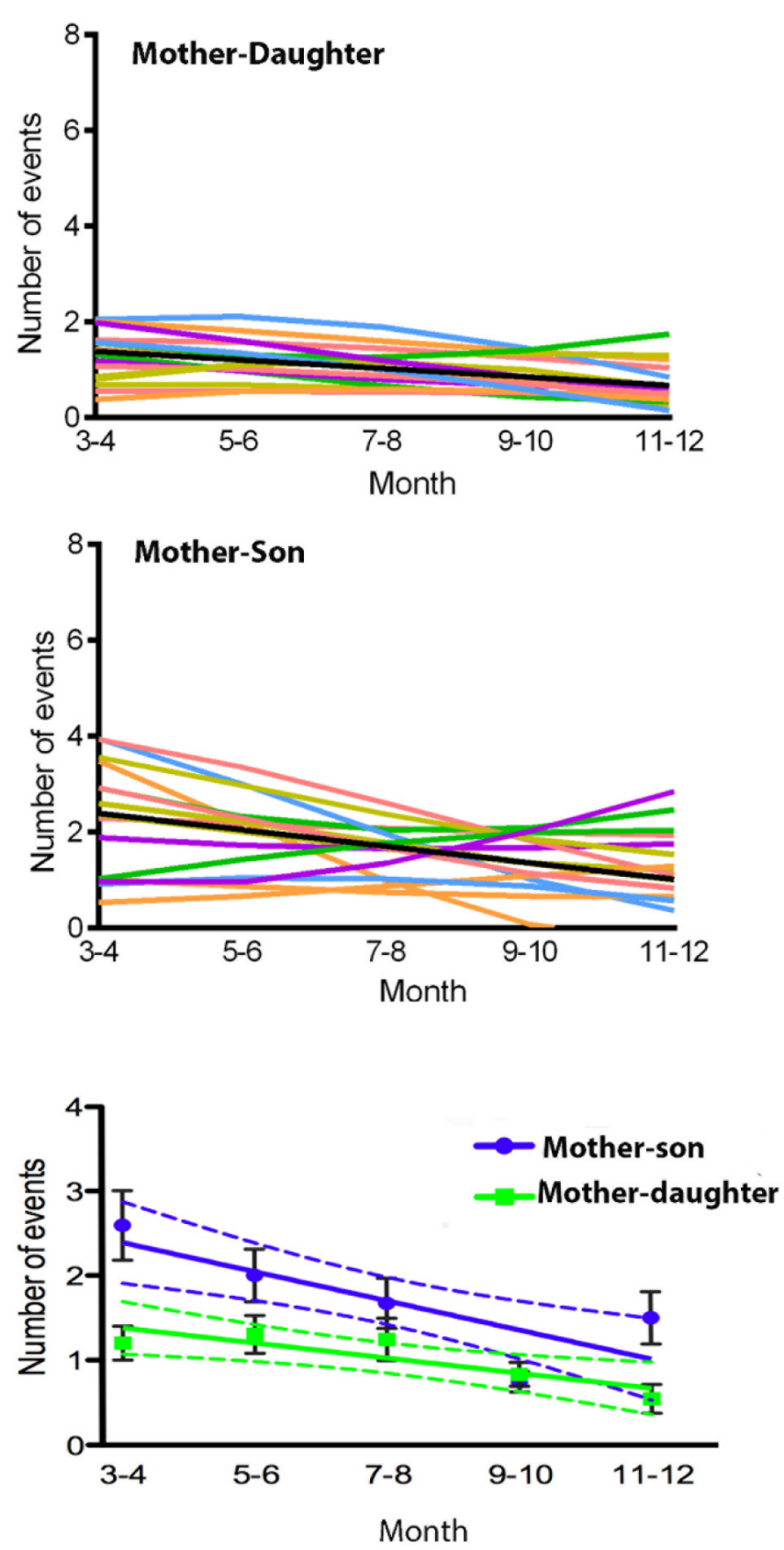

Figure 8: Sit-assisted mother. Frequency in 2-Month groups from 3-12 months. Top panel shows individual dyads with girls (thin colored lines) and a regression line for dyads with girls (thicker black line). Middle panel shows individual dyads with boys (thin colored lines) and a regression line for dyads with boys (thicker black line). The bottom panel shows group regression lines and standard deviations (Square symbol, green line = mother-daughter dyads; round symbol-blue line = mother-son dyads). 
$(, 1,147)=6.94, p=.009$. For frequency of affectionate touch events the pattern of change did not differ for mothers of sons versus daughters $F(1,146)=0.02, p=.90$, slope for sons $=-0.40$ and for daughters $=-0.42$. However, the $y$-intercepts for these regression lines were marginally significant $F(1,147)=4.08 ; p=.05, y$-intercept for sons $=$ 5.29 and for daughters $=4.69$. For duration of affectionate touch events the pattern of change also did not differ for mothers of sons compared to daughters $F(1,146)=$ $0.13, p=.72$, slope for sons $=-1.81$ and for daughters $=-1.19$. However, the elevation (y-intercepts) for these regression lines differed significantly $F(1,147)=7.58, p=$ .007, intercept 28.84 for sons, 17.84 for daughters. For the frequency of vocalization events neither the developmental pattern (slope) $F(1,146)=2.18, p=.14$, slope for sons $=0.11$ and for daughters $=0.89$ nor the $y$-intercepts $F$ $(1,147)=1.45, p=.23, y$-intercept for sons $=26.17$ and for daughters $=18.88$ differed based on gender/sex of infant. For vocalization duration neither the slopes $F(1,136)=1.13$, $p=.29$, slope for sons $=-2.55$ and for daughters $=0.84$ nor the $y$-intercept sons $=147.33$, daughters $=128.33$ differed using 2-month time bins $F(1,137)=0.27, p=.60$.

We used three-dimensional scatter plots and mesh plots to get a combined look at the above behaviors and, with the scatterplots, to gain a sense of individual dyad variability. Figures 9 (Months 3-4) and 10 (Months 5-6) show 3D scatterplots and mesh plots mapping the duration of maternal vocalization, assist locomotion and affectionate touch for dyads with girls and dyads with boys. For each of these figures, the $x$-axis represents assisted locomotion, the $y$-axis represents maternal vocalization and the z-axis affectionate touch. We have used the same scale in all five figures to facilitate visual comparison.

In Figure 9A (scatterplot) it appears as if the behavioral patterns of mothers of sons cluster towards the higher end of the $x$-axis (assisted locomotion) but spread out along the $y$ - (vocalization) axis. The pattern for mothers of daughters seems almost complementary. Also visible are two outlying dyads with greater levels of affectionate touch. For one of these, a mother did not move her daughter from one place to another at all, but offered a great deal of affectionate touch, and talked a lot to her daughter. For the other, a mother-son dyad, the mother was neither very affectionate, nor did she assist locomotion very much, but she vocalized to her son for longer durations. When we use the same data to draw a 3D mesh (Figure 9B) it appears that as populations, the mothers of sons and mothers of daughters occupy overlapping, but clearly different 3D state spaces.
The two "wings" of the mesh (one for the mother-son mesh and the other for the mother daughter mesh) connect to the data points from the outlying dyads. Examined together, the two graphs suggest that for this age period the statespace occupied by these three behaviors is more restricted for mothers of sons, compared to mothers of daughters. By 5-6 months (Figure 10), the state-space occupied by the mother daughter dyads is reduced, with almost total overlap between mother-son and mother-daughter populations, a pattern which persists thereafter (see additional graphs in Appendix 2).

\section{Clustering Comparison of Mother-son and Mother- daughter Dyads}

Visually, especially for months three to four, mother-son and mother-daughter dyads seem to occupy differentalbeit overlapping-- three-dimensional spaces for assisting locomotion, vocalizing and offering affection. To examine these impressions more systematically, we used K-means cluster analysis. $\mathrm{K}$-cluster analysis is a data-mining technique that uses nearest neighbor analysis (Wagstaff, Cardie, Rogers, \& Schroedl, 2001). Using SPSS, we specified a search for three clusters to be discovered for the combined data (all thirty dyads), using 10 iterations. We used the test's ANOVA function to assess the importance of each factor (assist locomotion, vocalization and affectionate touch) for cluster formation (Laerd Statistics, 2015). After initial cluster formation and membership identification for the 30 pooled dyads (i.e. without regard to gender/sex of infant), we determined by gender/sex of the child, which dyads belonged to which cluster. To illustrate the method, we present event duration only.

In Table 6, we present the number of cases in each cluster, and indicate which of the three factors contributed significantly to the clustering result. We considered a contribution to be significant whenever $p \leq .05$ in the ANOVA table produced with the K-means cluster command. Maternal vocalization contributed significantly to cluster formation for all of the month periods. In addition, assist locomotion contributed to four of the five periods (all save five to six months) while affectionate touch only contributed significantly for the 11-12-month period. In two periods (months seven to eight and nine to ten), case membership in one of the clusters rose above $50 \%$ of total cases. For example, during months 7-8, 17 dyads (57\%) were in the lowest vocalization-- middle assist locomotion-affectionate touch state space. During months nine to ten, 16 dyads (53\%) fell into the highest affectionate touch, but intermediate vocalization and assist locomotion clusters. 

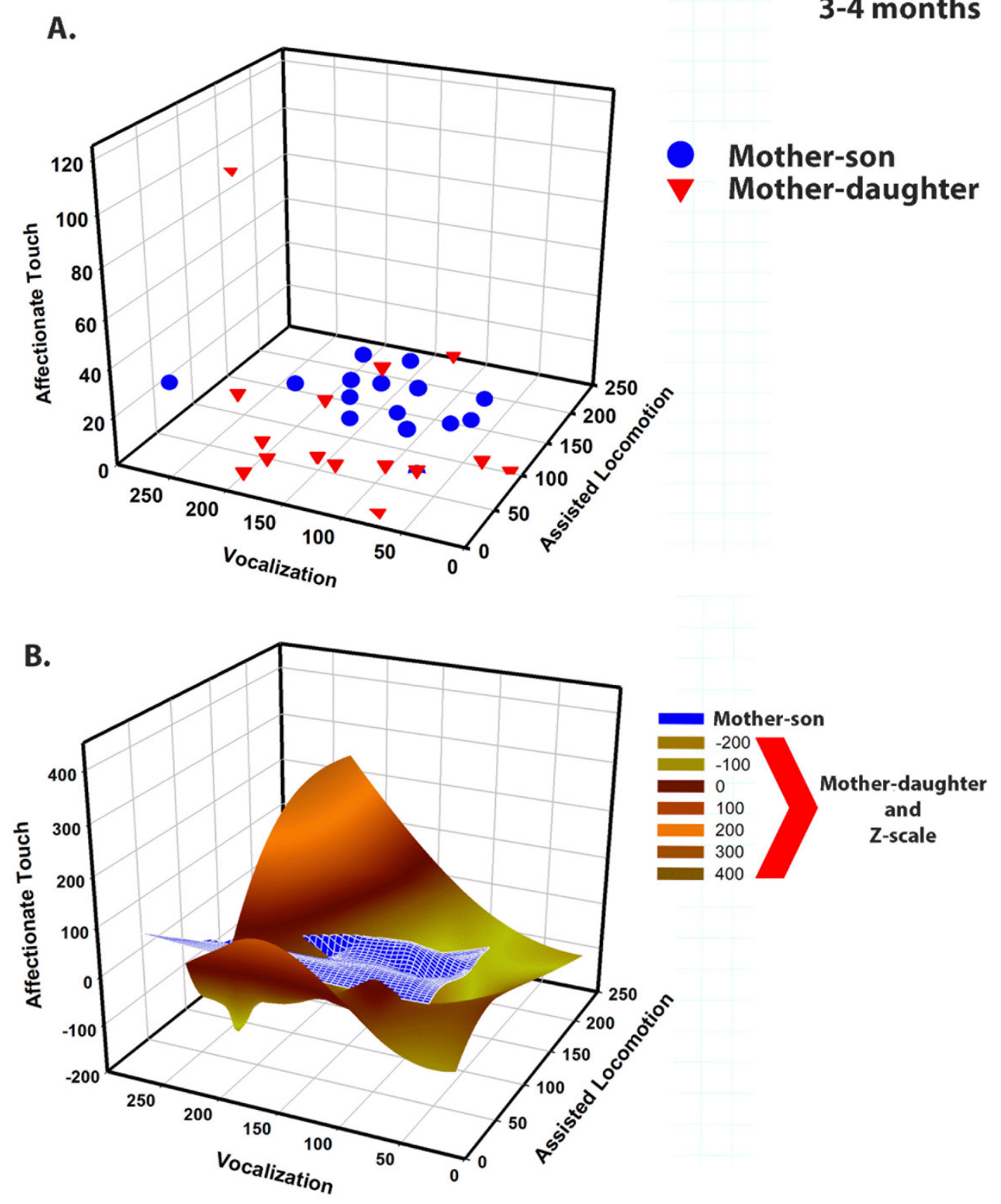

Figure 9: A scatterplot revealing the three-dimensional state space at 3-4 months for duration of the maternal behaviors assisted locomotion ( $x$-axis), vocalization ( $y$-axis) and affectionate touch (z-axis) divided by mothers of sons) and mothers of daughters. The blue circles and red triangles represent individual mother-infant dyads. In B: the mesh indicated as male is presented as single color with lines. The mesh indicated as female is presented as a gradient with the gradient scale indicative of z-axis values. 
A.

\section{5-6 months}

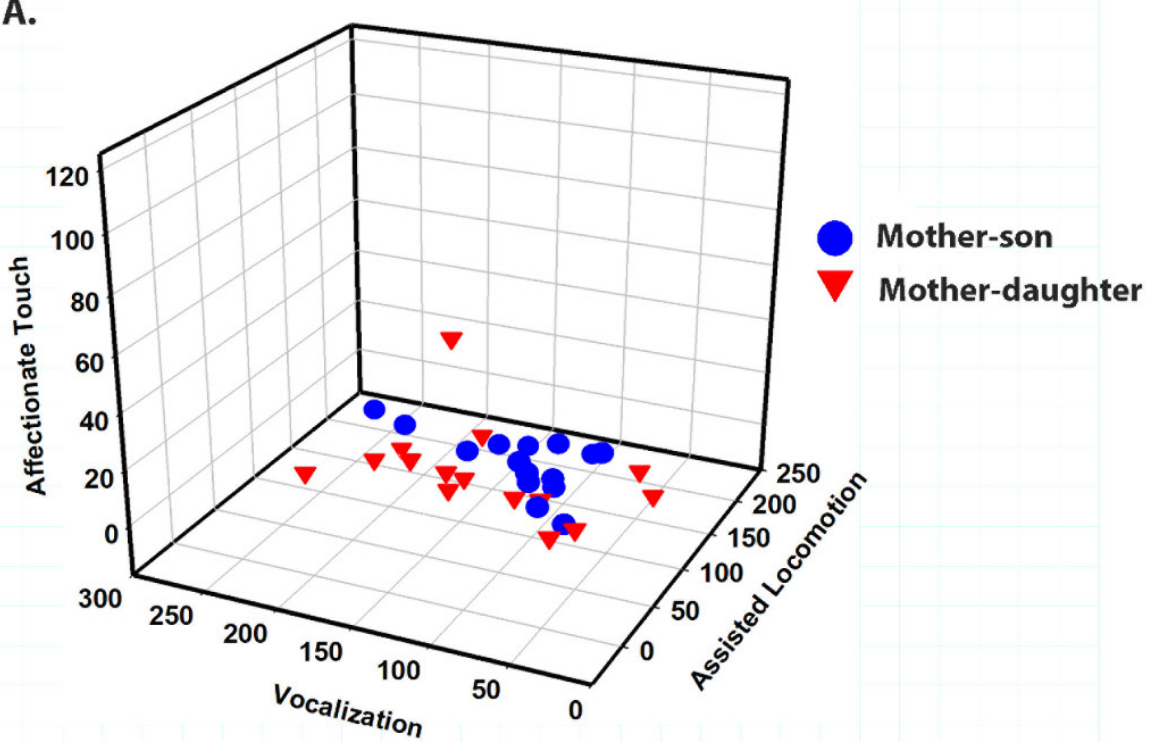

B.

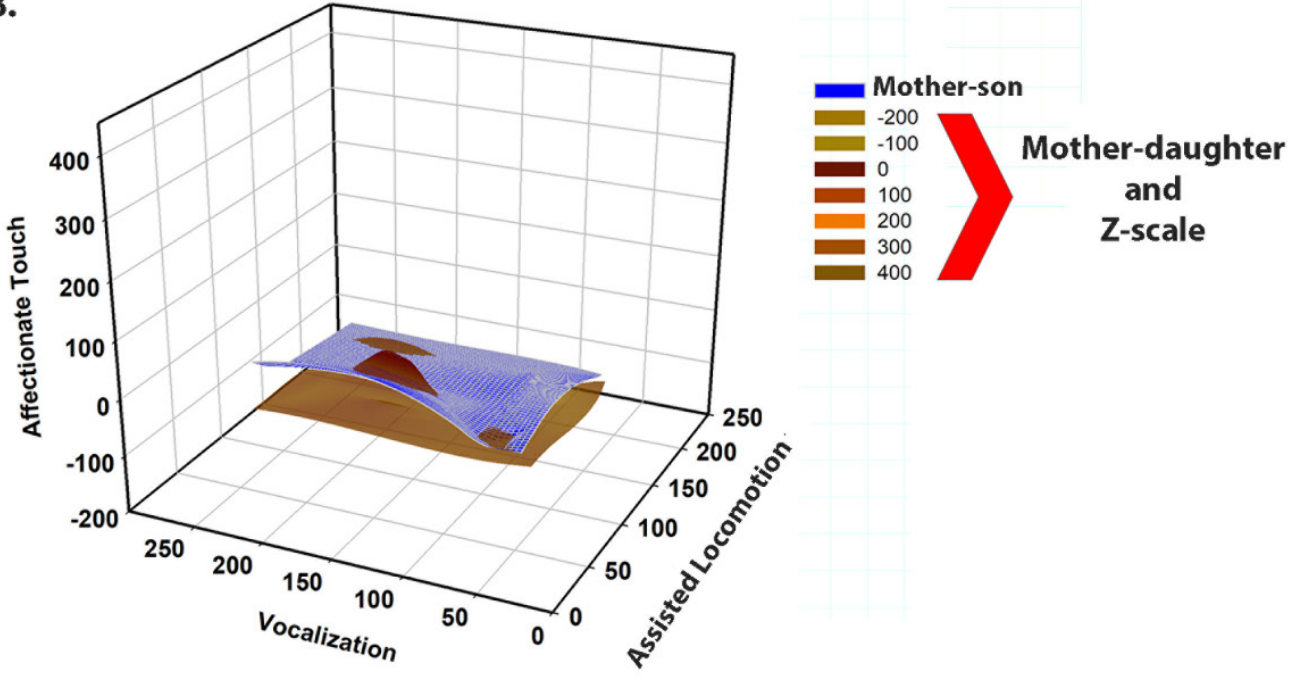

Figure 10: A. A scatterplot revealing the three-dimensional state space at 5-6 months for duration of the maternal behaviors assisted locomotion ( $x$-axis), vocalization ( $y$-axis) and affectionate touch ( $z$-axis) divided by mothers of sons and mothers of daughters. The blue circles and red triangles represent individual mother-infant dyads. In B: the mesh indicated as male is presented as single color with lines. The mesh indicated as female is presented as a gradient with the gradient scale indicative of $z$-axis values. 
Table 6

Factor Significance (ANOVA from K-means cluster) and Number of Cases for Each Cluster by Month Group

\begin{tabular}{|c|c|c|c|c|c|}
\hline Months & & $\begin{array}{l}\text { Significant } \\
\text { cluster } \\
\text { factor }\end{array}$ & $\begin{array}{l}\text { Cluster } \\
1 \text { (\# of } \\
\text { cases) }\end{array}$ & $\begin{array}{l}\text { Cluster } 2 \\
\text { (\# of } \\
\text { cases) }\end{array}$ & $\begin{array}{l}\text { Cluster } \\
3 \text { (\# of } \\
\text { cases) }\end{array}$ \\
\hline \multirow[t]{3}{*}{$3-4$} & Vocalization & Yes & & & \\
\hline & $\begin{array}{l}\text { Assist } \\
\text { Locomotion }\end{array}$ & Yes & 8 & 18 & 2 \\
\hline & $\begin{array}{l}\text { Affectionate } \\
\text { Touch }\end{array}$ & No & & & \\
\hline \multirow[t]{3}{*}{$5-6$} & Vocalization & Yes & & & \\
\hline & $\begin{array}{l}\text { Assist } \\
\text { Locomotion }\end{array}$ & No & 7 & 14 & 9 \\
\hline & $\begin{array}{l}\text { Affectionate } \\
\text { Touch }\end{array}$ & No & & & \\
\hline \multirow[t]{3}{*}{ 7-8 } & Vocalization & Yes & & & \\
\hline & $\begin{array}{l}\text { Assist } \\
\text { Locomotion }\end{array}$ & Yes & 16 & 7 & 7 \\
\hline & $\begin{array}{l}\text { Affectionate } \\
\text { Touch }\end{array}$ & No & & & \\
\hline \multirow[t]{3}{*}{$9-10$} & Vocalization & Yes & & & \\
\hline & $\begin{array}{l}\text { Assist } \\
\text { Locomotion }\end{array}$ & Yes & 12 & 5 & 12 \\
\hline & $\begin{array}{l}\text { Affectionate } \\
\text { Touch }\end{array}$ & No & & & \\
\hline \multirow[t]{3}{*}{$11-12$} & Vocalization & Yes & & & \\
\hline & $\begin{array}{l}\text { Assist } \\
\text { Locomotion }\end{array}$ & Yes & 13 & 9 & 7 \\
\hline & $\begin{array}{l}\text { Affectionate } \\
\text { Touch }\end{array}$ & Yes & & & \\
\hline
\end{tabular}


The main lesson to take from this portion of the clustering exercise is that although each dyad exhibited a unique multimodal pattern of interaction, the patterns can be aggregated into similar clusters.

To see whether the assignment of cases to particular clusters differed by infant gender/sex, we used a new variable, produced by the $\mathrm{K}$-means clustering operation, that indicates dyad gender/sex within each cluster for each month group. We performed a crosstabs operation for this variable with cluster membership in columns and sex in rows. In Figure 11 (three to four months) we present histograms of these distributions along with a table listing the cluster criteria in seconds for the three to four months group. The data for month groups five to six, seven to eight, nine to 10 and 11-12 may be found in Appendix 2. In Figure 11 , we see that maternal vocalization for cluster 1 is over twice the duration for clusters two or three. At the same time, cluster one contains the lowest values for duration of assisted locomotion. From the histogram we read that there are two mother-son dyads compared to six mother daughter dyads assigned to cluster one. A similar cluster of mother-daughter dyads in the low assisted locomotion/high vocalization group can also be seen in Figure 11. Figure 11 also shows that cluster numbers two and three have similar durations of vocalization, but that cluster three has more than two times longer durations for assisted locomotion. From the histogram we read that there are equal numbers of mother-son and mother-daughter dyads (two each). Only cluster two, which has intermediate durations for both vocalization and assisted locomotion has more mother-son (11) than mother-daughter (7) dyads.

Ideally, we would have liked to perform a Chi Square test on the distribution of cluster number by sex for each of the five-month groups. We could not, however, perform a valid test on the two-month groupings because once we divided each grouping into three clusters and then determined gender/sex membership within each cluster, our sample size became too small. To get some sense of whether mother-son and mother-daughter dyads distributed differently within clusters we used the information in Figure 11 and in Appendix 2 to array male and female counts according to membership in one of six groups: highest, intermediate and lowest vocalization duration, and highest, intermediate or lowest assisted/locomotion duration. This gave us high enough counts to perform Chi-Square tests on each of the two-month groups. Our null hypothesis was that membership in each cluster (high vocalization, intermediate vocalization, low vocalization etc.) would be the same for

\section{Final Cluster Centers 3-4 Months Duration (Seconds)}

\begin{tabular}{lr|r|r} 
& \multicolumn{3}{c}{ Cluster } \\
& \multicolumn{1}{c}{1} & \multicolumn{1}{c}{2} & \multicolumn{1}{c}{3} \\
\hline Maternal Vocalization & 197.61 & 87.82 & 86.96 \\
\hline Assisted Locomotion & 50.48 & 69.61 & 184.21 \\
\hline Affectionate Touch & 14.91 & 22.02 & 11.88 \\
\hline
\end{tabular}

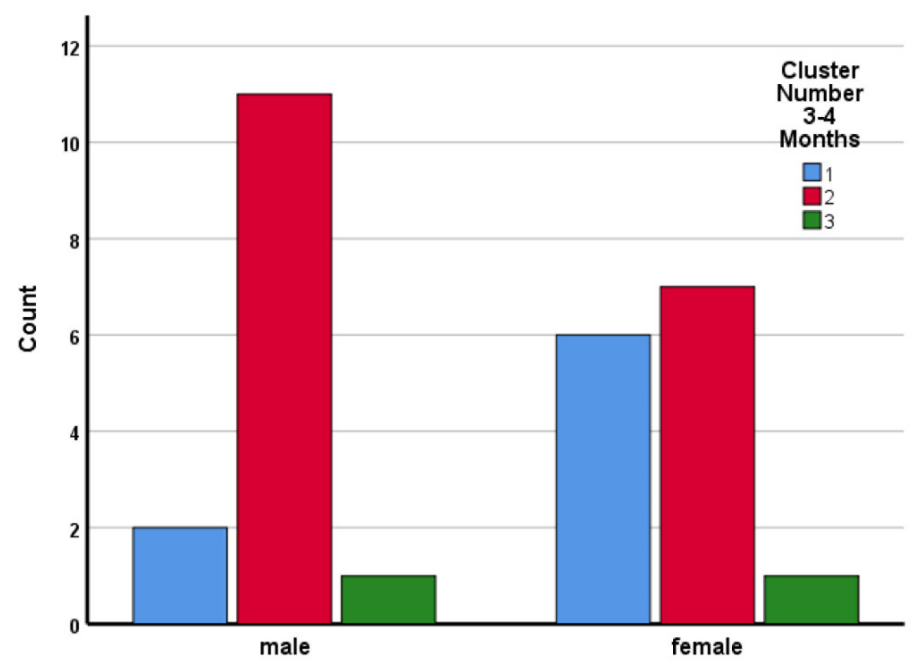

Figure 11: Final cluster centers: 3-4 months duration. The table lists average number of seconds per five-minute coding sequence for each of three maternal behaviors. The histogram indicates the number of dyads with male infants and the number of dyads with female infants in each of the clusters listed in the cluster table. 
dyads with boys and dyads with girls. The null hypothesis did not hold for the three to four-month olds, $\chi^{2}(5)=12.13$, $p=.03$, but was confirmed for the other time periods. Given this result, combined with the 3D scatterplots and meshes, we feel comfortable saying that a pattern for mother-daughter dyads that involves relatively longer periods of vocalization, but shorter periods of maternally assisted movement characterized the three to four month developmental period. This compares with relatively longer durations of assisted locomotion and less maternal vocalization for mother-son dyads. Affectionate touch did not seem to differ by gender/sex during the 3-4 month time period.

Finally, we used a simple correlation analysis on the entire sample (mother-son and mother-daughter dyads combined) to see whether high or low vocalization, assisted locomotion, or affectionate touch observed at three to four months correlated with the same behaviors at 11-12 months. The correlation between duration of maternal speech at three to four months and 11-12 months was 0.62. That for duration of assisted locomotion was 0.15 and for affectionate touch was -0.01 . One would expect less assisted locomotion as the infant becomes more independent, but the results suggest that patterns of affection in the early months do not carry over to later months. In contrast, maternal speech duration patterns within dyads are more consistent over the course of the first year of development.

\section{What are They Talking About? What are They Doing?}

The cluster analysis showed different patterns of talking while assisting locomotion for mother-son compared to mother-daughter dyads. Reading from Figure 9A, we see that at similar durations of speech (50-200 seconds), 10 mothers of daughters engaged in assisted locomotion for 100 seconds or less, while 14/15 of the mothers of sons engaged in such assistance for durations between 100 and 250 seconds. What was a mother saying as she supported her child's movement? Were there differences in the ways in which mothers supported motor movement depending on whether the infant was a boy or a girl?

To explore these questions, we looked in greater depth at maternally assisted standing, an aspect of assisted locomotion. We defined assisted standing as any three+ seconds during which the mother physically supported the infant in a standing position. As described in the Methods section and in Figure 3, we applied new codes to previously
A.

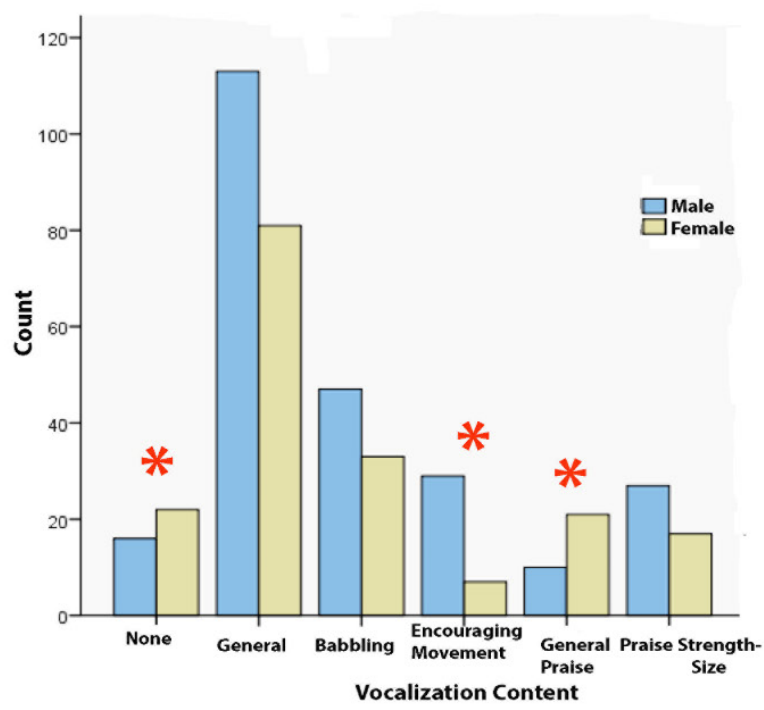

B.

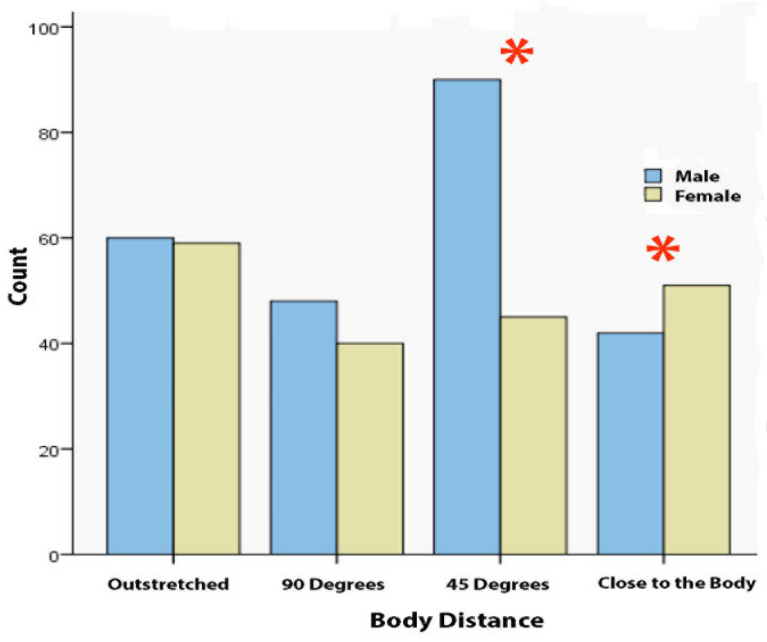

Figure 12: A. indicates the maternal vocalization content during assisted standing on a numerical scale for motherson compared to mother-daughter dyads, as defined in Chapter 3. The asterisk indicates significance at the $p$ $<.05$. B. indicates distance from mother's body during assisted standing as defined in Chapter 3 for mother-son compared to mother-daughter dyads. The asterisk indicates significance at the $p<.05$ 
identified assisted standing events using qualitative descriptors for vocalization and mode of physical support. We transcribed all vocalizations, and organized content into five categories: no vocalization, general, babbling, encouraging movement ("Do you want to walk? "You can do this!"), general praise ("Good job!" "Yes!"), and praise about body size/strength ("Look at those strong legs!"; "Look how strong you are!"). We observed 459 five-minute segments, with an average of 17 five-minute clips per dyad. There were more male maternal assisted standing events $(n=253)$ than female ones ( $n=226$ ), an average of 17.7 events per dyad. The number of events per dyad ranged from one to 33 $(S D=12.603)$.

Figure $12 \mathrm{~A}$ contains histograms comparing the frequency of vocalization content categories and 12B illustrates body distance categories by sex of the child. Using SPSS, we analyzed the results for vocalization and for body distance using an omnibus Chi-Square test of homogeneity, followed by a post hoc analysis using multiple $z$-tests of two proportions. Significant relationships were found between gender/sex and the frequency of categories of vocalization and body distance. For vocalization, $\chi^{2}(5, N$ $=423)=19.91, p=.001$. For body distance, $\chi^{2}(3, N=435)$ $=12.08, p=.007$.

Post hoc analysis involved pairwise comparisons of vocalization during assisted standing subcategories using multiple z-tests of two proportions with a Bonferroni correction. During assisted standing events, mothers were significantly less likely to vocalize to daughters than to sons (6.6 vs. $12.2 \%$ ). During vocalization, sons experienced significantly more episodes of vocal encouragement of movement (12.0 vs. $3.9 \%$ ), while girls experienced significantly more episodes of general praise (11.6 vs. $4.1 \%)$. The post hoc tests of body distance categories revealed two significant differences. Mothers held sons at a 45-degree arm angle more often than they did daughters (37.5 vs. 23.1\%). In contrast, mothers held daughters close to their bodies significantly more often than sons ( $26.2 \%$ vs. $17.5 \%$ ).

\section{Conclusion: wrapping things up}

We have divided this final section into three parts. First, we discuss our specific empirical results and relate them both to existing literature on early development, and to the general ideas set out in the previous essay sections entitled "Modeling Identity" and "Constructing a Timeline". Second, we introduce a more abstract model of gender/sex identity formation as a continuous set of processes. Finally, we propose a way forward for empirical researchers interested in forging new investigative paradigms that explore gender/ sex and gender/sex identity as a process rather than a trait or thing.

\section{Discussion of Reported Data}

\section{Dyads and Motor-related Behaviors}

The most striking gender/sex-related difference observed for maternal-infant play types involved the duration of motorsocial play, which describes instances in which dyadic play involved direct motor activities without any intervening objects. As seen in Table 5 and Figure 4, the developmental pattern (negative slope with increasing age of infant) for such play did not differ for dyads with boys compared to ones with girls. However, the elevation ( $y$-intercept) was of 1.35 times higher duration for dyads with boys, indicating longer periods of motor-social play at younger ages. This finding confirms the patterns of play interactions reported by Fogel, Garvey, Hsu and West-Stroming (2006, especially graphs on p. 107).

Motor-social play is a joint activity of mother and infant. If we were to use a Cartesian-split approach (Overton, 2015) to analyze our findings and those of Fogel et al., we would ask "what part does each individual play in starting and maintaining activity within a motor-social interaction?" Given that motor-social play is more common when the infant is younger and less capable of independent play initiation, it seems likely that in this early period the mothers take most of the initiative. However, mothers could be responding to different behaviors--too subtle for us to capture with our coding scheme--from boys compared to girls. Framing their research using Cartesian-split metatheory would lead researchers to employ additional methods, including a variety of non-invasive neural measures (Kuhl \& Rivera-Gaxiola, 2008), measurement of vagal tone (Feldman, 2015) and infant headcams (Smith, Jayaraman, Clerkin, \& Yu, 2018) to further examine the infant's world; similarly, researchers might probe the caregiver's point of view in these early months by in depth interviews, questionnaires, etc.

Relational/process/systems developmentalists might use the same measuring devices as Cartesians. Process theorists are, however, more interested in examining how dyadic patterns progress over time. For example, Fogel and colleagues provided evidence for three functions for what they call frames (what we have named play types)-historical, bridging and emerging. In their study of 13 dyads recorded weekly from four to 34 weeks of age, they determined that the motor-social frame was historical for nine of the dyads. 
That is, it was seen early in development and sometimes became the bridging behavior to a more stable object play (what we are calling child-directed play-in which the infant plays alone with an object but with maternal engagement at a distance). They also reported that four of their 13 dyads exhibited very low levels of motor-social play across their entire study period. Similarly, we observed a subset-all mother-daughter dyads-that showed low levels of motorsocial play for the entire observation period.

\section{Infant Behaviors}

The literature contains reports of sex-related differences in infant behavior. Moss (1967) observed that at both three weeks and three months male infants were fussier and more irritable than female infants. They were also more passive when awake, and at three weeks were more often supine, a result that is compatible with our findings that male infants more often were coded positive for the lie:still code (see Table 5 and Figure 7). Moss also noted that threeweek-old males had their eyes on the mother more often than did similarly aged females. This observation seems to contrast with a report by Connellan et al. (2000) that female neonates were more interested in human faces. Osofsky and O'Connell (1977) reported male newborns scored higher on measures of reactivity and females on responsivity and habituation. Fausto-Sterling et al. (2012a) summarized scattered reports, many of them single, unconfirmed findings, of sex-related differences in behavior in young infants.

In our results section, we report large sex-related differences in two infant behaviors, the frequency of infant lie:still and the frequency of infant sitting:assisted mother. Both had highly significant y-intercept differences (Table 5 and Figures 7 and 8). Unsurprisingly, the frequency of lying still or sitting with maternal assistance decreases with age, while sitting independently or standing assistedother increases with age. Both patterns presumably result from an overall improvement in motor skill as development progresses. Nevertheless, lie:still and sit:assisted mother exhibit gender/sex-related differences in frequency (y-intercept differences). The observed gender/sex differences in certain infant motor behaviors is of great interest, but they cannot be discussed without considering related maternal behaviors.

\section{Maternal Behaviors}

As shown in Table 5 there were $y$-intercept differences for three movement-related maternal behaviors. The first two were for the assist/shift behavior (Figure 5), which describes instances in which the mother repositions the infant without any obvious input from the infant. For both frequency and duration, slopes decreased with increasing age of the infant. However, the elevation (y-intercepts) for both the frequency and duration of assist/shift was significantly higher for mothers of boys. The second maternal behavior that differed in a highly significant manner for mothers of boys compared to mothers of girls was for the frequency of the motor code lift up (Table 5 and Figure 6). This code marked instances when the mother lifted the infant into the air as a form of play. For mothers of boys, the slope for frequency was steeper and declined rapidly, while for mothers of girls, lift up behavior occurred less often and the slope of the line with age was nearly flat. Given that on average boys weigh more at birth (see for example Australian Institute of Health and Welfare, 2000), we cannot attribute the longer duration of holding boys aloft to the possibility that they are lighter to lift. Lewis and Weinraub (1978) reported that in the first few months of infancy boys received more motor or touch stimulation (e.g. rocking and handling) while mothers talk and look more at girls.

Parental play stimulation differences according to infant sex in early infancy seem only rarely to have been studied (Zosuls \& Ruble, 2018). Instead, with the exception of Korner's analysis (1974), the existing literature focuses on bonding and dyad synchrony, and thus falls squarely within a relational-developmental systems meta approach. For example, Feldman (2003) studied levels of synchrony and patterns of arousal in mother-daughter, mother-son, fatherdaughter and father-son dyads in 5-month-old infants. She observed that same-sex dyads achieved greater synchrony than other-sex dyads. Fathers tended to play sessions that reached a peak of arousal one or more times per session. This contrasted with mothers, who in $44 \%$ of the play sessions with daughters and $35 \%$ for sons had no peak of arousal.

\section{Intra-dyad Interactions}

So far, we have discussed the dyad as a unit (play type) and each side (maternal or infant behaviors) of the dyadic equation. But how might specific behaviors influence one another? For example, after finding that boys lay still more frequently than girls, we explored the possibility that increased frequencies or durations of maternal motordirected behaviors involved a maternal response to infant inactivity. Using state-space grids we found that transitional entropy (TE), which is a measure of interactive organization, differed significantly for mother-son compared to motherdaughter dyads. High transitional entropy suggests less predictable interactions and lower scores indicate more 
organized or predictable interactions (Dishion et al., 2004; Hollenstein, 2013). Thus, the maternal response to boys lying still was less predictable than to girls.

\section{Dyads, Motor Behaviors, Speech Acts and Affectionate Touch}

Infants develop motor skills in part by processing sensoryespecially visual and tactile-input (Dusing, 2016; Williams et al., 2015). We asked whether there were gender/ sex differences in affectionate touch and speech during episodes of assisted locomotion. We first used 3D scatter and mesh plots grouped by gender/sex and age (Figures 9 and 10) to gain a visual impression of the differing patterns. Examining these 3D representations led us to explore further using K-means clustering (Table 6 and Figure 11). Assisted locomotion and vocalization were the main contributors to cluster membership except at 11-12 months, when affectionate touch also became important. At 3-4 months (Figure 11) and again at 11-12 months (Online Resource 2), more mother-daughter dyads belonged to clusters with high vocalization duration and low assisted locomotion duration. In contrast, at 3-4 months mother-son dyads most often appeared in clusters with intermediate durations of assisted locomotion and vocalization. ChiSquare analysis confirmed that cluster membership $X$ sex differed significantly at 3-4 months but not in the other time periods. Because our sample sizes became so small as we divided them into clusters and again by gender/sex we recommend that future analyses using these methods begin with a much larger sample size.

\section{Individual Differences}

A striking aspect of our longitudinal analyses was the array of individual family patterns. Consider, for example, the developmental shape of motor-social play in motherdaughter dyads. At one extreme (Figure 4), was a motherdaughter pair which virtually never engaged in motor play (an average of 10 seconds per five-minute sequence at three to four months declining to zero by nine to ten months). At the other, we observed a mother-daughter pair that averaged over 200 seconds in motor-social play, declining to 50 seconds per five-minute segment by months 11-12. For mother-son pairs, the divergence was also striking. For example, one dyad engaged in just over 50 seconds of motor-social play during months three to four, compared to a dyad engaging in about 190 seconds of motor-social play during months three to four. The wide range of individual patterns of behavior are also evident on the 3D scatter and mesh plots (Figures 9 and 10) that place families in a three- dimensional state space for maternal vocalization, assisted locomotion and affectionate touch.

The fact that behavior patterns for individual families are highly variable is unsurprising given the large number of factors that contribute to patterns of child rearing (a child's irritability, weight, and birth experience, and prior maternal experience with younger siblings or baby-sitting, maternal coping mechanisms and abilities, etc.). Thelen (2000b) noted that individual infants reach the same end state (e.g. reaching or walking) by different means. Thelen et al. (1993) emphasized physical constraints (e.g., body weight or postural position), as well as individual levels of physiological development (e.g., muscle tone and neuromuscular connectivity) as possible sources of variability. Fogel, Garvey, Hsu \& West-Stroming (2006) found that although dyads reached the endpoint of what they termed "not-guided object play" (infant playing independently with object with mother's attention), each dyad followed a different pathway to this common destination. They assumed that for infants still too young to move on their own or sit upright without support, "virtually all of the infant's exposure to objects occurred in a social context," and that the achievement of not-guided object play required an adult "who arranged the infant in proximity to objects and brought objects into infant reach space" (p. 223).

\section{General Data Characteristics: Strengths and Weaknesses}

A strength of the empirical component of this essay is the density of the longitudinal data-- second-by-second coding of five-minute segments of two recordings per month for nine months of in-home naturalistic interactions between mothers and infants detailing dozens of maternal and infant behaviors. However, the time-consuming nature of such a large coding effort dictated that we rely on a relatively small sample size (15 mother-son and 15 mother-daughter dyads). The fact that the data for each sex-group were often highly variable was both to be expected and a source of considerable interest. On the other hand, variability and relatively small sample size sometimes limited the statistical evaluation of group differences. (For further discussion of statistical concerns, see Appendix 1). Other weaknesses include the sample's lack of social and ethnic diversity, the absence of observations for the periods from birth to three months and 13 to 36 months, and the lack of interactions with siblings, peers, fathers or other caregivers. Future studies using newly collected samples can rectify each of these problems.

Our data remain relevant despite the fact that gender 
norms and behaviors have changed since 1989-1990 when the tapes we analyzed were recorded. Because much of the most frequently-cited literature on sex role and gender socialization stems from the same period (e.g. Fagot \& Hagan, 1991; Lytton \& Romney, 1991; Pomerantz \& Ruble, 1998), our analysis enhances our ability to interpret this still heavily-cited literature. We also suspect that our results retain significance for contemporary child-rearing practices. For example, Poulin-Dubois et al. (1994) reported that infants at nine months could distinguish matched adult female voices and faces, but it took until 12 months to acquire the same skill for adult male voices and faces. This timing is the same as that reported twenty years later by Boisferon et al. (2015). The fact that this result has not changed even with twenty years of cultural change is supported by a recent study of thirty racially mixed families, showing that $70 \%$ of the faces infants see during their first three months are female (Sugden, Mohamed-Ali, \& Moulson, 2014). Even if, just as in 1989-1990, the majority of primary caregivers in infancy are still women, it is possible that these caretakers no longer handle boy and girl infants differently (Eliot, 2009). This, however, strikes us as unlikely given the renewed emphasis on identifying the sex of the child even before birth. Historical evidence suggests that the more equal public gender roles have become, the greater the tendency to mark the sex of the infant (Paoletti, 1987; 1997). If, nevertheless, the subtle, day-to-day handling of, and interactions with, male and female infants have changed in the past twenty years, this study still provides a historical baseline and a theoretical framing for future comparisons.

\section{An Embodiment Model (To be a Girl or a Boy? That is the Question).}

Embodied gender/sex and gender/sex identity are both relational and individual phenomena. Thelen, for example, focused on an individual child's relationship between the physicality of the body and the physical world with which it interacts. The cognitive and affective development of gender/sex (identity), however, are inseparable from an infant's interactions with other humans. Varela's model of the co-emergence of autonomous selfhood and the world (Varela, 1997) examines the back and forth between the autonomous individual operating within a social world. Gender/sex contains a contradiction between autonomous and social identity. On the one hand, from birth through about age five, identity usually becomes stable and autonomous. On the other, acquiring both autonomy and a self-defined gender/sex requires profound interactions with others (Raczaszek-Leonardi, Nomikou, \& Rohlfing, 2013). Many infancy researchers emphasized that during an infant's first six months the caregiver-infant dyad forms a single developmental unit (Beebe et al., 2000; Beebe et al., 1997; Feldman, Golan, Hirschler-Guttenberg, Ostfeld-Etzion, \& Zagoory-Sharon, 2014; Feldman, et al., 2006; Fogel, Garvey, Hsu, \& West-Stroming, 2006). One of the infant's initial tasks is to absorb bodily information as a subunit of the dyad, and with such information, gradually bud off as an autonomous individual.

In Figure 13, we present a model, based on the ideas developed by Varela (1997), of the underlying interrelationships that create and maintain gender/sex identities. In this model, individual identities involve interactive domains. Thus, a child cannot arrive at a stable sense of self as boy or girl (Upper left-hand ellipse quadrant: Identity) without engaging in dyadic interactions, and specific sorts of gender/sex-specified activities (Upper right-hand ellipse quadrant: Domain of Interactions). At the same time, self-identity in the autonomous individual (Left side: Autonomous individual insert) requires larger-world interactions that produce contextualized meanings about gender/sex (Right side: Individual in interaction with others and objects insert). As indicated by the large top arrow that links the Individual with the World, individuals cannot separate or articulate an understanding of self, outside of their location in the world's meanings.

Such contextualized meanings may be what others refer to as gender schema (Liben \& Signorella, 1980; Martin $\&$ Halverson, 1981). For example, "girls are people who play with dolls" emerges as a generally known fact or a component of a gender schema. The domain of interactions (Figure 13. Upper right ellipse quadrant) starts early with the absorption of bodily information as a subunit of the dyad (Beebe \& Lachmann, 1994). Over time, the interactive domain expands to include interactions such as choice of clothing and toys as well as peer interactions. These social interactions gain significance (Figure 13. lower right quadrant) as others, through words and actions, interpret them as gender/sex. As infants observe positive, negative, or neutral valences attached to their own and others' gender/ sex representations, they develop what Thompson calls an intentional link (see extensive discussions of intentionality in Thompson, 2007). One set of intentional behaviors, focused on by some gender/sex developmentalists is selfsocialization, understood as a child's active efforts to match their own behaviors to perceived standards (Zosuls, Ruble, \& 
Tamis-Lemonda, 2014; Zosuls et al., 2009).

The emergence of intentional behavior moves the child from the domain of "Significance in the World" to the lower left quadrant of Figure 13: "operational closure in the individual". In terms of gender/sex, we define operational closure as the multi-month process during which children acquire linguistic labels, the ability first to label gender/sex of self and others passively, then actively and over time to acquire the concepts of gender/sex constancy and gender/ sex stability (Bem, 1989; Fagot \& Hagan, 1991; Fagot, Hagan, Leinbach, \& Kronsberg, 1985; Fagot \& Kavanagh, 1993). According to Varela, operational closure gives rise to a global property (what we call identity) without requiring "a central controller" such as an identity gene or a special group of identity cells in the brain.

Operational closure stabilizes the underlying processes that maintain gender/sex identity, thus allowing individuals to establish themselves as autonomous beings. We see gender/sex identity as an autopoietic system, that is, a network that reproduces itself, "and that also regulates the boundary conditions necessary for its ongoing existence as a network" (Bourgine \& Stewart, 2004, p. 327). Establishing autopoesis completes the circular flow by stabilizing individual identity (Figure 13: upper left quadrant. As constituted by the flow of events in Figure 13, identity is at once a property of the individual body/mind and a collective property involving interactions with others and with objects in the world. In the metatheoretical language introduced earlier in this essay, while identity may appear to be a "thing", it is actually a stable set of processes. Its development and continued maintenance and shaping depends on underlying activities that are both autonomous and intersubjective.

We can summarize this abstract model by considering the common view of "properly" gender-identified boys and girls. The stereotypical boy runs around shooting laser guns (or whatever) and engages socially by chasing and running. The stereotypical girl plays more quietly and engages in face-to-face social activities. Children in these idealized categories prefer different clothes that seemingly suit their activity patterns as well as their fickle preferences (Maccoby, 1998). Through these physical and interactive presentations, that vary continuously rather than in the stereotypical binary fashion so often presented, they come to understand themselves as a boy or a girl. They reinforce a blooming sense of identity by the very activities and codes of dress and conduct that led them to self-label in the first place. By the time children are three to four years old gender/sex has become autopoietic and self-socialized (Thompson, 2007; Varela, 1996; Zosuls \& Ruble, 2018).

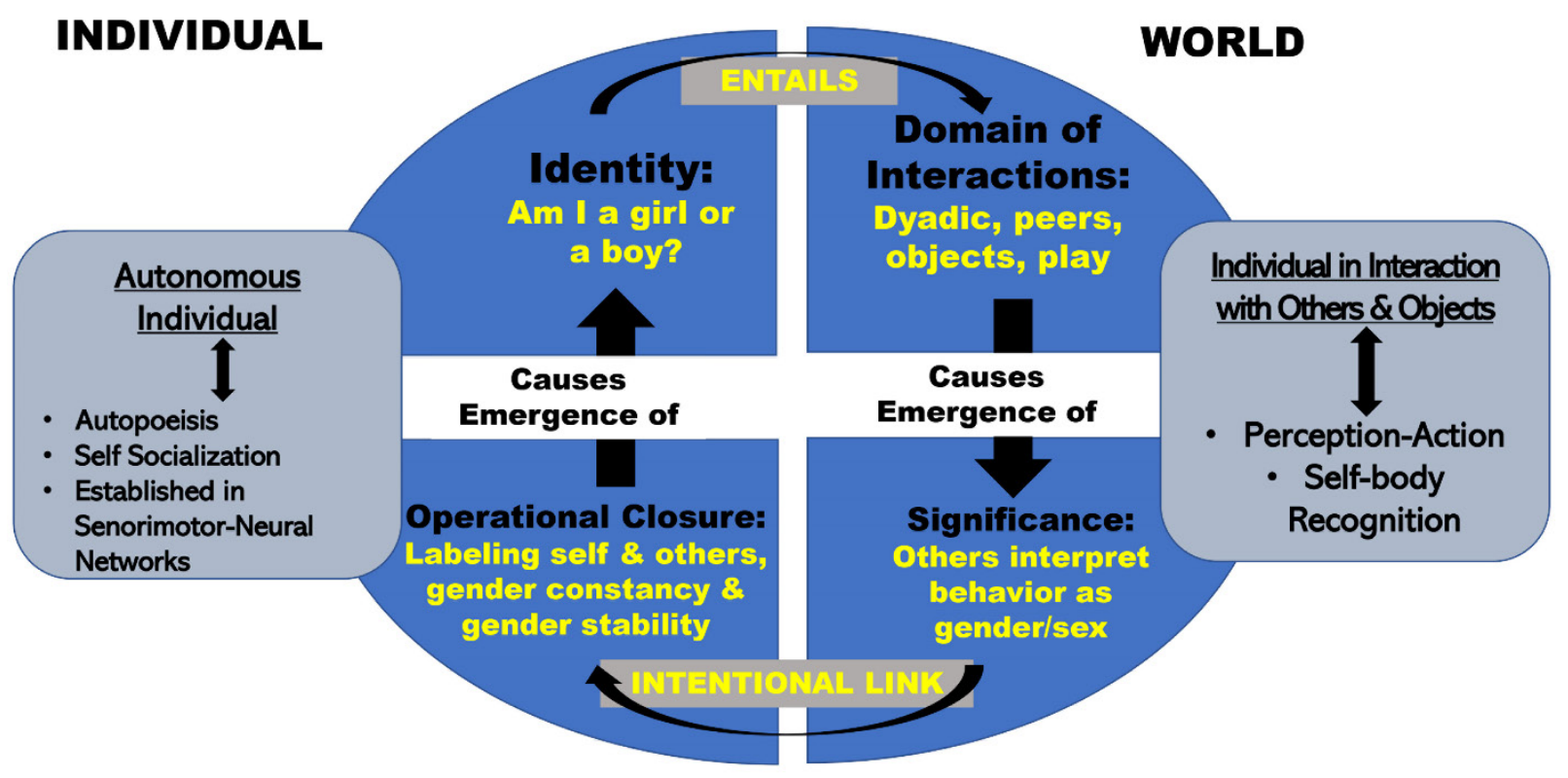

Figure 13: Model of embodied development and gender/sex. See text for extended description. 
As it develops, the path to gender/sex identity travels through and integrates into the body. We demonstrated several differences in dyadic behavior between mothers and their daughters compared with mothers and their sons. We believe these behavioral clusters contribute importantly to an infant's physical sense of self and immerse them within an existing cultural pattern of gender that the infant integrates into her or his emerging gender/sex identity. We have found differences in behaviors related to developing motor and body sense (dyadic distance, verbal emphasis on body size and strength, and more or less maternally aided motor workouts). Others have reported dyadic differences in patterns of vocalizing (Ahl et al., 2013; Sung et al., 2013), object play (de Barbaro et al., 2013; Landerholm \& Scriven, 1981), clothing, and types of toys offered and generally available (Paoletti, 1987, 1997; Todd et al., 2016; Wu et al., 2011; Zosuls et al., 2009).

In other words, at the same time that infants are learning to recognize gender/sex differences in their surroundings, they are literally weaving gender/sex into their neuro-muscular capacities and related neural routines into their connectomes (Yap et al., 2011). Furthermore, individual differences in infant responses to the same stimuli may magnify or dampen effects from adult-child exchanges. During the first two years an infant's neuromuscular and sensorimotor repertoires narrow, focus, and link to gender/sex in the world. Daily, moment-to-moment dyadic interactions are the crucial intermediaries connecting developing neural networks to "gender-in-the-world". Finally, the neural networks that mediate "gender-in-theworld" reverberate as gender/sex identity in the individual mind/body of the emergent toddler.

Our model is compatible with the idea that gender/sex expression and identity are interlaced continua. As a culture, through a variety of institutions, we usually force gender/ sex identity and expression into a social and structural binary. For example, we only offer two possibilities on a birth certificate, two types of bathrooms, and until recently children had only two identity options-boy or girl. In order to fit a continuum into a binary structure, researchers produced the concepts "gender non-conforming" or "gender variant". We hypothesize instead that the range of individual infant, parent and infant-parent dyad differences in motor (and probably other) behaviors-such as those reported on this study-- shapes a range of gender/sex embodiment. Such shaping ultimately feeds into the stream of information out of which identity itself coalesces. If this is so, the behaviors currently labeled and measured as "gender non-conforming, gender variant, gender atypical or gender incongruent" simply fall among a number of possible gender/sex identities (Zucker \& Wood, 2011; Drescher, Cohn-Kettenis \& Reed, 2016).

\section{Future Directions}

\section{Expanding the Field's Approach}

Though detailed and informative, the results presented here are just a first step toward an empirically supported developmental account of embodied gender/sex. Theoretical approaches need to accommodate individual and cultural diversity. Thus, we encourage future researchers to study more ethnically and economically diverse families as well as less conventional families, for example, families with a single parent or with same sex parents. Our findings and theoretical framing offer information that will enable future researchers to increase sample size and diversity by streamlining the coding, focusing on behaviors that we have already shown to be of possible interest. Newer methods exist for automating some aspects of the analysis (Johnson, Caskey, Rand, Tucker, \& Vohr, 2014; Roy et al., 2006), and for more precisely describing interactions through the use of both infant and caregiver head cameras or multiple angle camera shoots. In new analyses we would want to track historical, bridging and newly emerging interactive dyadic frames (play types) and integrate how dyads incorporate socio-cultural aspects of gender into their relational play patterns (Fogel, Garvey, Hsu \& West-Stroming 2006).

\section{Infant Components}

Our aspirations cannot be achieved with a single larger study. We suggest that an interdisciplinary consortium for the process-relational study of gender/sex development is in order. We need more information about individual features that contribute to a child's developmental pattern. These include birthweight, Apgar scores, length of gestation, type of delivery (cesarean, with or with anesthetic, prematurity), circumcision, and vagal tone at birth (see the work of Feldman, 2006; Feldman, 2008) and (Dipietro et al., 2010; Dipietro \& Voegtline, 2015).

Especially in the early months, infants have relatively few codeable behaviors. It is, however, possible to gather the sort of information just mentioned and, while studying infants interacting dyadically, including the use of physiological measures (Mcfarland, Fortin, \& Polka, 2019) and non-invasive brain scan techniques (Kuhl 
\& Rivera-Gaxiola, 2008). With innovative use of data from head cameras it would also be possible to capture what each dyad member is seeing in any particular interaction.

\section{Caregiver and Ecosystem Components}

The proposed interdisciplinary consortium must include studies that are ethnically and sexuality-diverse, because gender/sex is not identically embodied in all cultures (see results and literature discussion in Lew-Levy et al., 2019). Our current study sample controlled for maternal experience by choosing only first-time mothers. First-time mothers, however, may have been single children, or may have come from large families in which the study participant had already gained considerable infant care experience. It might also make sense to recruit families with more than one child so that prior experience and sibling effects could be explored (Rust, Golombok, Hines, Johnston, \& Golding, 2000). A richer group of studies would administer questionnaires about parental gender attitudes and take a regular census of toy and clothing types and colors, as well as bedroom and play area décor, thus providing data about the gender/sex-related ecosystem in which caregiver-infant dyads interact.

New studies should use an expanded time frame, Ideally, data collection should begin during the first month after birth and continue through three years of age. By extending to the third year we could observe the emergence of a range of gender/sex identities, perhaps developing measures that offer nuance beyond the traditional binary, i.e. incorporating identities that many currently label as non-conforming. Thereafter, follow-ups aimed at assessing developing gender identity, gender roles and gender expression should occur at five and 10 years. Such long-term studies are rare but those that exist are richly informative (Feldman, 2015; Li, Kung, \& Hines, 2017).

\section{Methods}

It should go without saying that the study of gender/ sex embodiment requires detailed longitudinal, dataintense investigations of the sort Lavelli et al. (2008) call microgenetic (Lavelli, Pantoja, Hsu, Messinger, \& Fogel, 2008). Microgenetic studies of dyadic interaction should include observations of diapering, dressing, and bathing, as these involve intimate moments of touch, instruction, and commentary on infant appearance and responsiveness. We further urge the use of graphing methods such as those illustrated in our results section, so that researchers can visualize and communicate complex change on the page as well as in the mind's eye. For example, with a larger data set collected from birth to three years, it would be revealing to visualize the three-dimensional state space of hypothesized gender/sex-related behavioral interactions of infants, toddlers and caregivers. Our graphing methods allowed us to look at individual variation and population differences and similarities at the same time, with the potential to understand gender/sex as continuous variation both within and between groups and as a combined function of several modalities.

We close, then, by repeating the developmental systems mantra: look first at the period before a phenomenon of interest is measurable, record details of the phenomenon during its emergence, and describe and account for the phenomenon's emergence and stabilization as a consistent and measurable skill or personality feature. To this we add: embodied gender/sex identity is both an individual property and a feature of the world that envelopes each person. As researchers we need to be alert to the ways in which each stabilizes and also changes the other.

\section{Acknowledgements}

This research was supported by funds from the Ford Foundation and the Pembroke Center for Teaching and Research on Women awarded to Anne Fausto-Sterling. The authors thank Dr. Ronald Seifer for the use of videotapes he originally recorded in order to study infant temperament. We also thank Dr. Cynthia García Coll for her collaboration and essential advice during the early years of this project, especially as she mentored a non-psychologist in the ways of the field of research psychology. We thank Dr. Tom Hollenstein of Queen's University, Kingston, Ontario for generous advice over a period of several years in the use of State Space Grids. Dr. Damian Kelty-Smith generously helped us with the post-hoc analysis of sample size. Our data result from the tireless coding efforts carried out over a number of years of a large group of Brown University undergraduates. Without them there would be no data!

Different versions of this publication underwent thorough peer review. We thank the anonymous peer reviews for their input; the resulting product responded substantially to their suggestions.

The watercolor figures were commissioned by the first author (who retains the copyright) from artist Thea Ernest. Any use of her artwork, even in the classroom or informal talks, should acknowledge her (\#TheaErnestDesign), and copyright questions should be addressed to the first author. 


\section{References}

Ahl, R. E., Fausto-Sterling, A., García Coll, C., \& Seifer, R. (2013). Gender and discipline in 5-12-month-old infants: A longitudinal study. Infant Behavior and Development, 36, 199-209. doi:10.1016/j.infbeh.2013.01.005

Australian Institute of Health and Welfare, National Perinatal and. Statistics Unit. (2000). Australia's mothers and babies (12). Retrieved from Canberra: http://www.npsu.unsw.edu.au/ ps12high.htm

Bailey, J. M., Vasey, P. L., Diamond, L. M., Breedlove, S. M., Vilain, E., \& Epprecht, M. (2016). Sexual orientation, controversy, and science. Psychological Science in the Public Interest, 17, 45-101. doi:10.1177/1529100616637616

Bakeman, R., \& Adamson, L. B. (1984). Coordinating attention to people and objects in mother-infant and peer-infant interaction. Child Development, 55, 1278-1289. doi: 10.2307/1129997https://www.jstor.org/stable/1129997

Balas, B., Saville, A., \& Schmidt, J. (2018). Neural sensitivity to natural texture statistics in infancy. Developmental Psychobiology, 60, 765-774. doi:10.1002/dev.21764

Beebe, B., Jaffe, J., Lachmann, F., Feldstein, S., Crown, C., \& Jasnow, M. (2000). Systems models in development and psychoanalysis: The case of vocal rhythm coordination and attachment. Infant Mental Health Journal, 21, 99-122. doi:10.1002/(SICI)10970355(200001/04)21:1/2<99::AID-IMHJ11>3.0.CO;2-\#

Beebe, B., Jaffe, J., Markese, S., Buck, K., Chen, H., Cohen, P., . . . Feldstein, S. (2010). The origins of 12-month attachment: A microanalysis of 4-month mother-infant interaction. Attachment and Human Development, 12, 3-141. doi:919621246 [pii]10.1080/14616730903338985

Beebe, B., \& Lachmann, F. (2002). Infant research and adult treatments: Co-constructing interactions (Kindle ed.). Hillside, NJ: The Analytic Press.

Beebe, B., \& Lachmann, F. M. (1994). Representation and internalization in infancy: Three principles of salience. Psychoanalytic Psychology, 11, 127-165. doi:http://dx.doi. org/10.1037/h0079530

Beebe, B., Lachmann, F. M., \& Jaffe, J. (1997). Mother-infant interaction structures and presymbolic self-and object representations. Psychoanalytic Dialogues, 7, 133-182. doi:10.1080/10481889709539172

Bem, S. L. (1989). Genital knowledge and gender constancy in preschool children. Child Development, 60, 649-662. doi:10.1111/j.1467-8624.1989.tb02745.x

Blakemore, J. E. O., Berenbaum, S. A., \& Liben, L. S. (2009). Gender development. New York: Psychology Press.

Boisferon, A. H. d., Dupierrix, E., Quinn, P. C., Lœvenbruck, H., Lewkowicz, D. J., Lee, K., \& Pascalis, O. (2015). Perception of multisensory gender coherence in 6- and 9-month-old infants. Infancy, 20, 661-674. doi:10.1111/infa.12088
Bourgine, P., \& Stewart, J. (2004). Autopoesis and cognition. Artificial Life, 10, 327-345.

Bussey, K., \& Bandura, A. (1999). Social cognitive theory of gender development and differentiation. Psychological Review, 106, 676-713. doi:http://dx.doi.org/10.1037/0033295X.106.4.676

Byrge, L., Sporns, O., \& Smith, L. B. (2014). Developmental process emerges from extended brain-body-behavior networks. Trends in Cognitive Sciences, 18, 395-403. doi:https://doi. org/10.1016/j.tics.2014.04.010

Byrne, A. (2019). What is gender identity? The elusive true gender self. In: Arcdigital media. https://arcdigital.media/what-isgender-identity-10ce0da71999

Campbell, D. W., \& Eaton, W. O. (1999). Sex differences in the activity level of infants. Infant and Child Development, 8, 1-17. doi:10.1002/(SICI)1522-7219(199903)8:1<1::AIDICD186>3.0

Campos, J. J., Anderson, D. I., Barbu-Roth, M. A., Hubbard, E. M., Hertenstein, M. J., \& Witherington, D. (2000). Travel broadens the mind. Infancy, 1, 149-219. doi: 10.1207/ S15327078in0102_1

Cao, M., He, Y., Dai, Z., Liao, X., Jeon, T., Ouyang, M., .. . Huang, H. (2017). Early development of functional network segregation revealed by connectomic analysis of the preterm human brain. Cerebral Cortex, 27, 1949-1963. doi:10.1093/cercor/bhw038

Cao, M., Huang, H., \& He, Y. (2017). Developmental connectomics from infancy through early childhood. Trends in Neurosciences, 40, 494-506. doi:http://dx.doi. org/10.1016/j.tins.2017.06.003

Casey, B. J., Tottenham, N., Liston, C., \& Durston, S. (2005). Imaging the developing brain: What have we learned about cognitive development? Trends in Cognitive Sciences, 9, 104-110. doi:10.1016/j.tics.2005.01.011

Clerkin, E. M., Hart, E., Rehg, J. M., Yu, C., \& Smith, L. B. (2017). Real-world visual statistics and infants' first-learned object names. Philosophical Transactions of the Royal Society B: Biological Sciences, 372. doi:10.1098/ rstb.2016.0055

Connellan, J., Baron-Cohen, S., Wheelwright, S., Batki, A., \& Ahluwalia, J. (2000). Sex differences in human neonatal social perception. Infant Behavior and Development, 23, 113-118. doi:https://doi.org/10.1016/S01636383(00)00032-1

Deaux, K. (1993). Commentary: Sorry, wrong number--a reply to Gentile's call. Psychological Science, 4, 125-126. doi:https://doi.org/10.1111/j.1467-9280.1993.tb00474.x

de Barbaro, K., Johnson, C. M., \& Deák, G. O. (2013). Twelvemonth "social revolution" emerges from motherinfant sensorimotor coordination: A longitudinal investigation. Human Development, 56, 223-248. doi: $10.1159 / 000351313$ 
Diamond, L. M. (2007). A dynamical systems approach to the develoment and expression of female same-sex sexuality. Perspectives in Psychological Science, 2, 142-161. doi: 10.1111/j.1745-6916.2007.00034.x

DiPietro, J. A., Kivlighan, K. T., Costigan, K. A., Rubin, S. E., Shiffler, D. E., Henderson, J. L., \& Pillion, J. P. (2010). Prenatal antecedents of newborn neurological maturation. Child Development, 81, 115-130. doi:10.1111/j.14678624.2009.01384.x

DiPietro, J. A., \& Voegtline, K. M. (2015). The gestational foundation of sex differences in development and vulnerability. Neuroscience. doi:http://dx.doi. org/10.1016/j.neuroscience.2015.07.068

Dishion, T. J., Bullock, B. M., \& Granic, I. (2002). Pragmatism in modeling peer influence: Dynamics, outcomes, and change processes. Developmental Psychopathology, 14, 969-981. doi:https://doi.org/10.1017/ $\underline{\mathrm{S} 0954579402004169}$

Dishion, T. J., Nelson, S. E., Winter, C. E., \& Bullock, B. M. (2004). Adolescent friendship as a dynamic system: Entropy and deviance in the etiology and course of male antisocial behavior. Journal of Abnormal Child Psychology, 32, 651663. doi:10.1023/b:jacp.0000047213.31812.21

Downey, G. (2009). Throwing like a girl's (brain). Retrieved from http://neuroanthropology.net/2009/02/01/throwing-likea-girls-brain/

Drescher, J., Cohen-Kettenis, P. T., \& Reed, G. M. (2016). Removal of gender incongruence of childhood diagnostic category: A human rights perspective - authors' reply. The Lancet Psychiatry, 3, 406. doi:10.1016/s2215-0366(16)30038-4

Dupré, J., \& Nicholson, D. (2018). Manifest of a processual philosophy of biology. In D. J. Nicholson \& J. Dupré (Eds.), Everything flows: Towards a processual philosophy of biology (pp. 1-38). Oxford: Oxford University Press.

Dusing, S. C. (2016). Postural variability and sensorimotor development in infancy. Developmental Medicine \& Child Neurology, 58, 17-21. doi:10.1111/dmcn.13045

Eichstedt, J. A., Serbin, L. A., Poulin-Dubois, D., \& Sen, M. G. (2002). Of bears and men: Infants' knowledge of conventional and metaphorical gender stereotypes. Infant Behavior and Development, 25, 296-310. doi:https://doi. org/10.1016/S0163-6383(02)00081-4

Eliot, L. (2009). Pink brain, blue brain. Boston: Houghton, Mifflin, Harcourt.

Fabes, R. A., Martin, C. L., \& Hanish, L. D. (2003). Young children's play qualities in same-, other-, and mixed-sex peer groups. Child Development, 74, 921-932. https://doi. org/10.1111/1467-8624.00576
Fabes, R. A., Martin, C. L., Hanish, L. D., Anders, M. C., \& MaddenDerdich, D. A. (2003). Early school competence: The roles of sex-segregated play and effortful control. Developmental Psychology, 39, 848-858. http://dx.doi. org/10.1037/0012-1649.39.5.848

Fagot, B. I., \& Hagan, R. (1985). Aggression in toddlers - responses to the assertive acts of boys and girls. Sex Roles, 12, 341351. doi: $10.1007 /$ Bf00287600

Fagot, B. I., \& Hagan, R. (1991). Observations of parent reactions to sex-stereotyped behaviors: Age and sex effects. Child Development, 62, 617. doi: 10.1111/j.1467-8624.1991. tb01556.x

Fagot, B. I., Hagan, R., Leinbach, M. D., \& Kronsberg, S. (1985). Differential reactions to assertive and communicative acts of toddler boys and girls. Child Development, 56, 1499. doi:10.2307/1130468

Fagot, B. I., \& Kavanagh, K. (1993). Parenting during the second year: Effects of children's age, sex, and attachment classification. Child Development, 64, 258-271. DOI:10.1111/i.1467-8624.1993.tb02908.x

Fagot, B. I., \& Leinbach, M. D. (1993). Gender-role development in young children: From discrimination to labeling. Developmental Review, 13, 205-224. doi: 10.1006/ drev.1993.1009

Fausto-Sterling, A. (2012). Sex/gender: Biology in a social world. New York: Routledge.

Fausto-Sterling, A. (2019). Gender/sex, sexual orientation, and identity are in the body: How did they get there? Journal of Sex Research, 56, 529-555. doi:10.1080/00224499.20 19.1581883

Fausto-Sterling, A., Crews, D., Sung, J., García Coll, C., \& Seifer, R. (2015). Multimodal sex-related differences in infant and in infant-directed maternal behaviors during months three through twelve of development. Developmental Psychology, 51, 1351-1366. doi:10.1037/dev0000033

Fausto-Sterling, A., García Coll, C., \& Lamarre, M. (2012a). Sexing the baby: Part 1- what do we really know about sex differentiation in the first year of life? Social Science and Medicine, 74, 1684-1692. doi:10.1016/j. socscimed.2011.05.051

Fausto-Sterling, A., García Coll, C., \& Lamarre, M. (2012b). Sexing the baby: Part 2 applying dynamic systems theory to the emergences of sex-related differences in infants and toddlers. Social Science and Medicine, 74, 1693-1702. doi: 10.1016/j.socscimed.2011.06.027

Feldman, R. (2003). Infant-mother and infant-father synchrony: The coregulation of positive arousal. Infant Mental Health Journal, 24, 1-23. doi:doi:10.1002/imhj.10041

Feldman, R. (2006). From biological rhythms to social rhythms: Physiological precursors of mother-infant synchrony. Developmental Psychology, 42, 175-188. doi:10.1037/0012-1649.42.1.175 
Feldman, R. (2008). The intrauterine environment, temperament, and development: Including the biological foundations of individual differences in the study of psychopathology and wellness. Journal of the American Academy of Child Adolescent Psychiatry, 47, 233-235. doi:10.1097/ CHI.0b013e3181613a92

Feldman, R. (2015). Mutual influences between child emotion regulation and parent-child reciprocity support development across the first 10 years of life: Implications for developmental psychopathology. Developmental Psychopathology, 27, 1007-1023. doi:10.1017/ S0954579415000656

Feldman, R., Masalha, S., \& Alony, D. (2006). Microregulatory patterns of family interactions: Cultural pathways to toddlers' self-regulation. Journal of Family Psychology, 20, 614-623. doi:10.1037/0893-3200.20.4.614

Feldman, R., Golan, O., Hirschler-Guttenberg, Y., OstfeldEtzion, S., \& Zagoory-Sharon, O. (2014). Parent-child interaction and oxytocin production in pre-schoolers with autism spectrum disorder. British Journal of Psychiatry. doi:10.1192/bjp.bp.113.13751

Ferber, S. G., Feldman, R., \& Makhoul, I. R. (2008). The development of maternal touch across the first year of life. Early Human Development, 84, 363-370. doi:10.1016/j.earlhumdev.2007.09.019

Fogel, A., Dickson, L., Hsu, H.-c., Messinger, D., Nelson-Goens, G. C., \& Nwokah, E. (1997). Communication of smiling and laughter in mother-infant play: Research on emotion from a dynamic systems perspective. New Directions in child Development, 77, 5-24. doi: 10.1002/cd.23219977702

Fogel, A., Garvey, A., Hsu, H.-C., \& West-Stroming, D. (2006). Change processes in relationships: A relational-historical research approach. Cambridge: Cambridge University Press.

Giedd, J. N., Castellanos, F. X., Rajapakse, J. C., Vaituzis, A. C., \& Rapoport, J. L. (1997). Sexual dimorphism of the developing human brain. Progress in Neuropsychopharmacology and Biological Psychiatry, 21, 1185-1201. doi:S0278584697001589 [pii]

Gliga, T. (2018). Telling apart motor noise from exploratory behaviour, in early development. Froniers in Psychology, 9, 1-9. doi:https://doi.org/10.3389/fpsyg.2018.01939

Gogtay N, Giedd JN, Lusk L, Hayashi KM, Greenstein D, Vaituzis AC, ... PM, T. (2004). Dynamic mapping of human cortical development during childhood through early adulthood. Proceedings of the American Academy of Science, 101, 8174-8179. doi: 10.1073/pnas.0402680101
Gottlieb, G. (1996). Developmental psychobiological theory. In R. B. Cairns, G. H. Elder, \& E. J. Costello (Eds.), Developmental science (pp. 63-77). Cambridge, UK: Cambridge University Press.

Gottlieb, G. (1997). Synthesizing nature-nurture: Prenatal roots of instinctive behavior. Mahwah: Lawrence Erlbaum.

Gottlieb, G., Wahlsten, D., \& Lickliter, R. (1998). The significance of biology for human development: A developmental psychobiological systems view. In W. Damon (Ed.), Handbook of child psychology (5th ed., Vol. 1, pp. 210257): John Wiley \& Sons, Inc.

Harris, A. (2005). Gender as soft assembly. Hillsdale: The Analytic Press.

Haslanger, S. A. (2012). Resisting reality: Social construction and social critique. New York: Oxford University Press.

HealthyChildren.Org. (2015). Gender identity development in children. Retrieved from https://www.healthychildren.org/ English/ages-stages/gradeschool/Pages/Gender-Identityand-Gender-Confusion-In-Children.aspx $1 /$

Higgins, J. (2018). Biosocial selfhood: Overcoming the 'body-social problem' within the individuation of the human self. Phenomenology and the Cognitive Sciences, 17, 433-454. doi:10.1007/s11097-017-9514-2

Hollenstein, T. (2007). State space grids: Analyzing dynamics across development. International Journal of Behavioral Development, 31, 384-396. doi:10.1177/0165025407077765

Hollenstein, T. (2013). State space grids depicting dynamics across development. In. New York : Springer.

Huston, A. C. (1983). Sex-typing. In P. H. Mussen \& E. M. Hetherington (Eds.), Handbook of child psychology (Vol. 4, pp. 387-468). NewYork: Wiley.

Hyde, J. S., Bigler, R., Joel, D., Tate, C., \& van Anders, S. M. (2018). The future of sex and gender in psychology: Five challenges to the gender binary. American Psychologist. doi:doi: 10.1037/amp0000307

Jansen, R. G., Wiertz, L. F., Meyer, E. S., \& Noldus, L. P. J. J. (2003). Reliability analysis of observational data: Problems, solutions, and software implementation. Behavior Research Methods, Instruments, \& Computers, 35, 391-399. doi:10.3758/bf03195516

Joel, D., \& McCarthy, M. M. (2016). Incorporating sex as a biological variable in neuropsychiatric research: Where are we now and where should we be? Neuropsychopharmacology. doi:10.1038/npp.2016.79 
Joel, D., Persico, A., Salhov, M., Berman, Z., Oligschläger, S., Meilijson, I., \& Averbuch, A. (2018). Analysis of human brain structure reveals that the brain "types" typical of males are also typical of females, and vice versa. Frontiers in human neuroscience, 12. doi:10.3389/ fnhum.2018.00399

Johnson, K., Caskey, M., Rand, K., Tucker, R., \& Vohr, B. (2014). Gender differences in adult-infant communication in the first months of life. Pediatrics, 134, e1603-1610. doi:10.1542/peds.2013-4289

Jordan-Young, R. M. (2010). Brainstorm: The flaws in the science of sex differences. Cambridge, MA.: Harvard University Press.

Kagan, J. (1994). Galen's prophecy: Temperament in human nature. New York: Basic Books.

Kohlberg, L. (1966). A cognitive-developmental analysis of children's sex-role concepts and attitudes. In E. E. Maccoby (Ed.), The development of sex differences (pp. 82172). Stanford, California: Stanford University Press.

Korner, A. F. (1974). Methodological considerations in studying sex differences in the behavioral functioning of newborns. In Friedman, Richard C.; Richart, Ralph M. et al. (Eds.) (1974). Sex differences in behavior.( pp. 197-208) Oxford, England: John Wiley \& Sons.

Kuhl, P., \& Rivera-Gaxiola, M. (2008). Neural substrates of language acquisition. Annual Review of Neuroscience, 31, 511-534. doi:10.1146/annurev.neuro.30.051606.094321

Kuhl, P. K. (2010). Brain mechanisms in early language acquisition. Neuron, 67, 713-727. doi:10.1016/j.neuron.2010.08.038

Kuhl, P. K., Stevens, E., Hayashi, A., Deguchi, T., Kiritani, S., \& Iverson, P. (2006). Infants show a facilitation effect for native language phonetic perception between 6 and 12 months. Developmental Science, 9, F13-F21. doi:10.1111/ j.1467-7687.2006.00468.x

Kuhl, P. K., Tsao, F.-M., Liu, H.-M., Zhang, Y., \& De Boer, B. (2001). Language/culture/mind/brain. Annals of the New York Academy of Sciences, 935, 136-174. doi:10.1111/j.1749-6632.2001.tb03478.x

Laerd Statistics (2015). Statistical tutorials and software guides. https://statistics.laerd.com/

Landerholm, E. J., \& Scriven, G. (1981). A comparison of mother and father interaction with their six-month-old male and female infants. Early Child Development and Care, 7, 317321. doi:10.1080/0300443810070405

Lavelli, M., Pantoja, A. P. F., Hsu, H.-C., Messinger, D., \& Fogel, A. (2008). Using microgenetic designs to study change processes. In Handbook of research methods in developmental science (pp. 40-65): Blackwell Publishing Ltd.

Legrand, D. (2006). The bodily self: The sensori-motor roots of pre-reflective self-consciousness. Phenomenology and the Cognitive Sciences, 5, 89-118. doi:10.1007/s11097-0059015-6
Lenroot, R. K., Gogtay, N., Greenstein, D. K., Wells, E. M., Wallace, G. L., Clasen, L. S., ... Giedd, J. N. (2007). Sexual dimorphism of brain developmental trajectories during childhood and adolescence. Neuroimage, 36, 10651073. doi:S1053-8119(07)00234-0 [pii]10.1016/j. neuroimage.2007.03.053

Lew-Levy, S., Boyette, A. H., Crittenden, A. N., Hewlett, B. S., \& Lamb, M. E. (2019). Gender-typed and gendersegregated play among tanzanian hadza and congolese bayaka hunter-gatherer children and adolescents. Child Development, 0. doi:10.1111/cdev.13306

Lewis, M., \& Weinraub, M. (1978). Sex of parent * sex of child: Socioemotional development. In R. C. Friedman, R. M. Richart, \& R. L. Vande Wiele (Eds.), Sex differences in behavior (pp. 165-190). Huntington, NY: Robert E. Krieger Publishing.

Lewkowicz, D. J., \& Ghazanfar, A. A. (2009). The emergence of multisensory systems through perceptual narrowing. Trends in Cognitive Sciences, 13, 470-478. doi:https://doi. org/10.1016/j.tics.2009.08.004

Lewkowicz, D. J., Schmuckler, M. A., \& Mangalindan, D. M. J. (2018). Learning of hierarchical serial patterns emerges in infancy. Developmental Psychobiology. doi:10.1002/ dev. 21614

Li, G., Kung, K. T. F., \& Hines, M. (2017). Childhood gender-typed behavior and adolescent sexual orientation: A longitudinal population-based study. Developmental Psychology, Advance online publication. doi:http://dx.doi.org/10.1037/ $\operatorname{dev} 0000281$

Liben, L. S., \& Signorella, M. L. (1980). Gender-related schemata and constructive memory in children. Child Development, 51, 11-18. doi:http://dx.doi.org/10.2307/1129584

Lytton, H., \& Romney, D. M. (1991). Parents' differential socialization of boys and girls: A metaanalysis. Psychological Bulletin, 109, 267-296. doi:doi:10.1037/0033-2909.109.2.267

Maccoby, E. E. (1998). The two sexes: Growing up apart: Coming together. Cambridge: Harvard University Press.

Manzouri, A., \& Savic, I. (2018). Possible neurobiological underpinnings of homosexuality and gender dysphoria. Cerebral Cortex, 29, 2084-2101. doi:10.1093/cercor/ bhy090

Mareschal, D., French, R. M., \& Quinn, P. C. (2000). A connectionist account of asymmetric category learning in early infancy. Developmental Psychology, 36, 635-645. doi:10.1037/0012-1649.36.5.635

Martin, C. L., \& Halverson, C. F. J. (1981). A schematic- processing model of sex typing and stereotyping in children. Child Development, 52, 1119-1134. doi: 10.2307/1129498.

Martin, C. L., \& Ruble, D. N. (2010). Patterns of gender development. Annual Review of Psychology, 61, 353-381. doi:10.1146/annurev.psych.093008.100511 
Martin, C. L., Ruble, D. N., \& Szkrybalo, J. (2004). Recognizing the centrality of gender identity and stereotype knowledge in gender development and moving toward theoretical integration: Reply to Bandura and Bussey (2004). Psychological Bulletin, 130, 702-710. doi:10.1037/00332909.130.5.702

McFarland, D. H., Fortin, A. J., \& Polka, L. (2019). Physiological measures of mother-infant interactional synchrony. Developmental Psychobiology, 0. doi:10.1002/dev.21913

Money, J., \& Ehrhardt, A. A. (1972). Man and woman, boy and girl. Baltimore: John's Hopkins University Press.

Moon, C., Lagercrantz, H., \& Kuhl, P. K. (2013). Language experienced in utero affects vowel perception after birth: A two-country study. Acta Paediatrica, 102, 156-160. doi:10.1111/apa.12098

Murray, M. M., Lewkowicz, D. J., Amedi, A., \& Wallace, M. T. (2016). Multisensory processes: A balancing act across the lifespan. Trends in Neurosciences, 39, 567-579. doi:http:// dx.doi.org/10.1016/j.tins.2016.05.003

Nishiyori, R., Bisconti, S., Meehan, S. K., \& Ulrich, B. D. (2016). Developmental changes in motor cortex activity as infants develop functional motor skills. Developmental Psychobiology, 58, 773-783. doi:10.1002/dev.21418

Osofsky, J. D., \& O'Connell, E. J. (1977). Patterning of newborn behavior in an urban population. Child Development, 48, 532-536. doi:10.2307/1128650

Overton, W. F. (2014). The process-relational paradigm and relational-developmental-systems metamodel as context. Research in Human Development, 11, 323-331. doi:10.108 0/15427609.2014.971549

Paoletti, J. B. (1987). Clothing and gender in america: Children's fashions 1890-1920. Signs, 13, 136-143. doi:10.1086/494390

Paoletti, J. B. (1997). The gendering of infants' and toddlers' clothing in america. In K. Martinez \& K. L. Amers (Eds.), The material culture of gender; the gender of material culture (pp. 27-35). Hanover: University Press of New England.

Pitts-Taylor, V. (2016). The brain's body: Neuroscience and corporeal politics Durham, NC: Duke University Press.

Pomerantz, E. M., \& Ruble, D. N. (1998). The role of maternal control in the development of sex differences in child self-evaluative factors. Child Development, 69, 458-478. doi:http://dx.doi.org/10.2307/1132178 ...

Poulin-Dubois, D., Serbin, L. A., Kenyon, B., \& Derbyshire, A. (1994). Infants' intermodal knowledge about gender. Developmental Psychology, 30, 436-442. doi:doi:10.1037/0012-1649.30.3.436

Raczaszek-Leonardi, J., Nomikou, I., \& Rohlfing, K. J. (2013). Young children's dialogical actions: The beginnings of purposeful intersubjectivity. Autonomous Mental Development, IEEE Transactions on, 5, 210-221. doi:10.1109/ TAMD.2013.2273258
Roy, D., Patel, R., DeCamp, P., Kubat, R., Fleischman, M., Roy, B., ... Gorniak, P. (2006). The human speechome project. Paper presented at the 28th Annual Conference of the Cognitive Science Society.

Ruble, D., \& Martin, C. L. (1998). Gender development. In N. Eisenberg (Ed.), Social, emotional and personality development (5th edition ed., Vol. 3, pp. 933-1016). New York: Wiley.

Ruble, D. N., Martin, C. L., \& Berenbaum, S. A. (2006). Gender development. In N. Eisenberg (Ed.), Handbook of child psychology (6th ed., Vol. 3, pp. 858-932). Hoboken: John Wiley and Sons.

Rust, J., Golombok, S., Hines, M., Johnston, K., \& Golding, J. (2000). The role of brothers and sisters in the gender development of preschool children. Journal of Experimental Child Psychology, 77, 292-303. doi:10.1006/ jecp.2000.2596S0022-0965(00)92596-7 [pii]

Sadjadi, S. (2019). Deep in the brain: Identity and authenticity in pediatric gender transition. Cultural Anthropology, 34, 103-129. doi:10.14506/ca34.1.10

Schlittler, R. (Producer). (2017). Definitions related to sexual orientation and gender diversity in apa documents. Retrieved from https://www.apa.org/pi/lgbt/resources/ sexuality-definitions.pdf

Seifer, R., Sameroff, A. J., Barrett, L. C., \& Krafchuk, E. (1994). Infant temperament measured by multiple observations and mother report. Child Development, 65, 1478-1490. doi:10.1111/j.1467-8624.1994.tb00830.x

Singer, J. D., \& Willett, J. B. (2003). Applied longitudinal data analysis: Modeling change and event occurrence. Oxford: Oxford University Press.

Smith, L. B. (2005). Cognition as a dynamic system: Principles from embodiment. Developmental Review, 25, 278-298. doi:10.1016/j.dr.2005.11.001

Smith, L. B., Jayaraman, S., Clerkin, E., \& Yu, C. (2018). The developing infant creates a curriculum for statistical learning. Trends in Cognitive Sciences, 22, P325-336. doi:https://doi.org/10.1016/j.tics.2018.02.004

Smith, L. B., Thelen, E., Titzer, R., \& McLin, D. (1999). Knowing in the context of acting: The task dynamics of the a-not-b error. Psychological Review, 106, 235-260. doi:10.1037/0033-295X.106.2.235

Spencer, J. P., Smith, L. B., \& Thelen, E. (2001). Tests of a dynamic systems account of the a-not-b error: The influence of prior experience on the spatial memory abilities of two-year-olds. Child Development, 72, 1327-1346. doi: 10.1111/1467-8624.00351

Spencer, J. P., Vereijken, B., Diedrich, F. J., \& Thelen, E. (2000). Posture and the emergence of manual skills. Developmental Science, 3, 216-233. doi:10.1111/14677687.00115 
Stern, D. E. (1985). The interpersonal world of the infant: A view from psychoanalysis and developmental psychology. New York: Basic Books.

Sugden, N. A., Mohamed-Ali, M. I., \& Moulson, M. C. (2014). I spy with my little eye: Typical, daily exposure to faces documented from a first-person infant perspective. Developmental Psychobiology, 56, 249-261. doi:10.1002/ dev.21183

Sung, J., Fausto-Sterling, A., Garcia Coll, C., \& Seifer, R. (2013). The dynamics of age and sex in the development of motherinfant vocal communication between 3 and 11 months. Infancy, 18, 1135-1158. doi:10.1111/infa.12019

Swaab, D. F., \& Hofman, M. A. (1995). Sexual differentiation of the human hypothalamus in relation to gender and sexual orientation. Trends in Neurosciences, 18, 264-270.

Thelen, E. (1995). Motor development: A new synthesis. American Psychologist, 50, 79-95. doi:10.1037/0003-066X.50.2.79

Thelen, E. (2000a). Grounded in the world: Developmental origins of the embodied mind. Infancy, 1, 3-28. doi: 10.1207/ S15327078IN0101_02

Thelen, E. (2000b). Many roads lead to rome: Locomotion and dynamics. Infancy, 1, 221-224. doi:10.1207/ S15327078IN0102_2

Thelen, E., Corbetta, D., Kamm, K., Spencer, J. P., Schneider, K., \& Zernicke, R. F. (1993). The transition to reaching: Mapping intention and intrinsic dynamics. Child Development, 64, 1058-1098. doi: 10.2307/1131327

Thelen, E., \& Smith, L. B. (1994). Dynamic systems: Exploring paradigms for change. In E. Thelen \& L. B. Smith (Eds.), A dynamic systems approach to the development of cognition and action (pp. xxiii, 376 p.). Cambridge, Mass.: MIT Press.

Thompson, E. (2005). Sensorimotor subjectivity and the enactive approach to experience. Phenomenology and the Cognitive Sciences, 4, 407-427. doi:10.1007/s11097-005-9003-x

Thompson, E. (2007). Mind in life: Biology, phenomenology, and the sciences of mind. Cambridge: Harvard University Press.

Thompson, E., \& Varela, F. J. (2001). Radical embodiment: Neural dynamics and consciousness. Trends in Cognitive Sciences, 5, 418-425. doi:https://doi.org/10.1016/S13646613(00)01750-2

Thompson, R. A., \& Nelson, C. A. (2001). Developmental science and the media: Early brain development. American Psychologist, 56, 5-15. doi:10.1037/0003-066X.56.1.5

Thorne, B. (1993). Gender play: Girls and boys in school. New Brunswick: Rutger's University Press.

Todd, B. K., Barry, J. A., \& Thommessen, S. A. O. (2016). Preferences for 'gender-typed' toys in boys and girls aged 9 to 32 months. Infant and Child Development, 26, e1986 doi:10.1002/icd.1986
Unger, R. K., \& Crawford, M. (1993). Commentary sex and gender-the troubled relationship between terms and concepts. Psychological Science, 4, 122-124. doi:http://dx.doi. org/10.1111/j.1467-9280.1993.tb00473.x ...

van Anders, S., \& Dunn, E. (2009). Are gonadal steroids linked with orgasm perceptions and sexual assertiveness in women and men? Hormones and Behavior, 56, 206-213. doi:10.1016/j.yhbeh.2009.04.007

van Anders, S. M. (2015). Beyond sexual orientation: Integrating gender/sex and diverse sexualities via sexual configurations theory. Archives of Sexual Behavior, 44, 1177-1214. doi:10.1007/s10508-015-0490-8

van Anders, S. M., Galupo, M. P., Irwin, J., Twist, M. L. C., \& Reynolds, C. J. (2019). Talking about transgender experiences, identities, and existences at conferences. Retrieved from https://docs.google.com/document/d/ 1iHodSA16oP0itTjZPkB5tsIBjMHOiMdy9lt9zmTPKPs/ edit?usp=sharing

Varela, F. J. (1996). Neurophenomenology: A methodological remedy for the hard problem. Journal of Consciousness Studies, 3, 330-349.

Varela, F. J. (1997). Patterns of life: Intertwining identity and cognition. Brain and Cognition, 34, 72-87. doi:https://doi. org/10.1006/brcg.1997.0907

Varela, F. J., Thompson, E., \& Rosch, E. (c1991). The embodied mind: Cognitive science and human experience. Cambridge, Mass.: MIT Press.

Vaughn, B. E., Contreras, J., \& Seifer, R. (1994). Short-term longitudinal study of maternal ratings of temperament in samples of children with down syndrome and children who are developing normally. American Journal of Mental Retardation, 98, 607-618.

von Eye, A., Bergman, L. R., \& Hsieh, C.-A. (2015). Person-oriented methodological approaches. In R. M. Lerner (Ed.), Handbook of child psychology and developmental science (7th ed., pp. 2-53). New York: John Wiley and Sons, Inc.

Wagstaff, K., Cardie, C., Rogers, S., \& Schroedl, S. (2001). Constrained k-means clustering with background knowledge. Proceedings of the Eighteenth International Conference on Machine Learning, 577-584.

West-Eberhard, M. J. (1989). Phenotypic plasticity and the origins of diversity. Annual Review of Ecological Systems, 20, 249-278. https://doi.org/10.1146/annurev. es.20.110189.001341

Williams, J. L., Corbetta, D., \& Guan, Y. (2015). Learning to reach with "sticky" or "non-sticky" mittens: A tale of developmental trajectories. Infant Behavior and Development, 38, 82-96. doi:http://dx.doi.org/10.1016/j. infbeh.2015.01.001 
Wu, R., Gopnik, A., Richardson, D. C., \& Kirkham, N. Z. (2011). Infants learn about objects from statistics and people. Developmental Psychology, 47, 1220-1229. doi:10.1037/ a0024023

Yap, P-T., Fan, Y., Chen, Y., Gilmore, J.H., Lin, W., \& Shen, D. (2011). Development trends of white matter connectivity in the first years of life. PLoS One, 6, e24678. doi:https://doi. org/10.1371/journal.pone.0024678

Young, I. M. (1990). Throwing like a girl and other essays in feminist philosophy and social theory. Bloomington: Indiana University Press.

Young, I. M. (1998). Throwing like a girl: Twenty years later. In D. Welton (Ed.), Body and flesh: A philosophical reader (pp. 286-290). Oxford: Blackwell.

Zosuls, K. M., \& Ruble, D. N. (2018). Gender-typed toy preferences among infants and toddlers. In E. S. W. L. M. Dinella (Ed.), Gender typing of children's toys: How early play experiences impact development (pp. 49-72). Washington, DC: American Psychological Association.

Zosuls, K. M., Ruble, D. N., \& Tamis-LeMonda, C. S. (2014). Selfsocialization of gender in African American, Dominican immigrant, and Mexican immigrant toddlers. Child Development, 85, 2202-2217. doi:10.1111/cdev.12261

Zosuls, K. M., Ruble, D. N., Tamis-Lemonda, C. S., Shrout, P. E., Bornstein, M. H., \& Greulich, F. K. (2009). The acquisition of gender labels in infancy: Implications for gender-typed play. Developmental Psychology, 45, 688-701. doi:200905916-007 [pii]10.1037/a0014053

Zucker, K. J., \& Wood, H. (2011). Assessment of gender variance in children. Child Adolesc Psychiatric Clinics of North America, 20, 665-680. doi:10.1016/j.chc.2011.07.006

\section{Appendix 1}

\section{Coding and Team Organization}

Infant and maternal behaviors and play type were coded on a second-by-second basis using Observer XT 7 software (Noldus Information Technology, Wageningen, Netherlands; https://www.noldus.com/the-observer-xt/gathering-data).

This software permits all of the coding to be entered on parallel timelines that can be visualized in the moment or exported for analysis with SPSS. We organized data collection by dyad identification number and accumulated coding sessions on separate USB drives with internal folders for weekly age at each visit. Coding was performed in passes by different coding teams, with two coders per team. One team was assigned play types; another team coded infant behaviors, while a third team coded maternal behaviors. Coders further specialized in behavior categories such as vocalization, or types of motor behavior. Coders who specialized in maternal gross motor-related behaviors, for example, could reopen the USB-stored file and code each of these behaviors as they started and stopped using the same timeline on which play type had been coded. This resulted in parallel timelines that allowed us to see which maternal or infant behaviors occurred during a particular play type. Data were exported to SPSS for separate analyses both as discrete event frequencies and event durations.

The coders were undergraduate students (about 15 total), coding in pairs over a period of about 3 years. Continuity first was ensured, by the supervision of a postdoctoral fellow (the second author of this paper) with extensive training in coding, and second, by appointing one of the experienced undergraduates as coordinator. During personnel transitions we ensured that one member of each coding pair was experienced. The new member had to reach reliability with the experienced member for data collection to proceed.

\section{Statistical Concerns}

\section{Sample Size}

At the time we planned and designed the project (2005), we followed the commonly-held belief that we needed a minimum sample size of thirty to obtain a data set with normal distributions (see discussion on https://www. researchgate.net/post/What is the rationale behind the_magic_number_30_in_statistics). We ran longitudinal analyses as described in the Methods section of the main paper for both duration and frequency for all of the motor-related play types, maternal behaviors and infant behaviors described in the Coding Manual, that is, upwards of 50 comparisons. Most of these showed no significant differences (with the standard $p \leq .05$ measure) in developmental pattern (slope) or elevation ( $y$-intercept). Because of concerns about the large number of comparisons made, we raised our criteria for significance, by emphasizing findings for which $p<.01$. We also performed a post hoc power analysis on the data used to test for group differences in this section. We used the method described by Efron $(2005$; 2013), who provides a computational method to test how replicable the results are under many variations in sampling. We performed 1000 re-samplings of the data (i.e., random selection with replacement of the same sample size). Across the resulting 1000 sets of coefficients, we found that all significant factors reported in this essay had coefficients greater than zero in more than 800 of these re-samplings (see also, Teng, Wallot \& KeltyStephen, 2016). We thus remain confident that despite 
Table A1-1.

Average Kappa Scores for Mother-Infant Play Type, Maternal, and Infant Behavior Codes

\begin{tabular}{lll}
\hline & Agreement & Average kappa score \\
\hline Mother-Infant Play Type & & \\
Joint object play & $88 \%$ & 0.76 \\
Motor-social & $81 \%$ & 0.76 \\
Child-directed object play & $72 \%$ & 0.49 \\
\hline Maternal Behaviors & & \\
Gross motor stimulation & $85 \%$ & 0.77 \\
Assist locomotion & $82 \%$ & 0.70 \\
Lift up & $88 \%$ & 0.80 \\
Infant-directed speech & $71 \%$ & 0.61 \\
Affectionate touch & $94 \%$ & 0.90 \\
\hline Infant Behaviors & & 0.75 \\
Standing & $94 \%$ & 0.81 \\
Sitting & $88 \%$ & 0.77 \\
Lying & $89 \%$ & 0.75 \\
Crawling & $88 \%$ & \\
\hline
\end{tabular}

the relatively small sample size necessitated by our laborintensive coding scheme, the study has enough power to support the conclusions we offer.

\section{Preregistration}

In a discussion of statistically valid experimental design in the field of psychology Wicherts, Veldkamp, Augusteijn, Bakker et al. (2016) provide a "Checklist to avoid p Hacking". They describe a number of design or process errors that can lead to statistically invalid inferences. Our choice to dig more deeply into the details of maternal behaviors during assisted standing seems to fall into their category D4, "Measuring additional constructs that could potentially act as primary outcomes" (Wicherts et al., 2016, Table 1, p. 3). We do not present the results of this sub-study as our primary outcome. Our choice to further explore the initial observations of assisted standing, however, produced results interesting enough to warrant a (future) carefully-designed study that compares gender/sex differences in body-distance holding, infant positioning, and vocalization content in mother/son compared to mother/ daughter dyads during infancy. Obtaining these sorts of data are essential for testing theories of gender/sex embodiment as a continuous process. 


\section{Coding Manual: Maternal Vocalization.}

Table A1-2

Maternal vocalization:overview (Code:subcode)

Speech
Tone
Neutral
Positive
Neutral/Negative
Content
Girl
Boy

Babble to Infant

Sing

Other Noise

\section{Definitions And Coding Directions}

Speech. The mother's coherent words, no matter where directed (to the infant, the camera person, or to herself), are coded as speech. The words need not be complete phrases. Even single words are coded as speech. If the utterance is identifiable as a word, even if it is not in proper English (e.g. "dipey" instead of "diaper"), it still falls under the category of speech. Animal noises that sound like words, or are otherwise word-like (i.e. "woof woof" or "moooo") should be coded as speech. Most sound-like 'words' (i.e. "wow," "whoa," "ho ho ho") are typically coded as "speech," but there are exceptions. In these cases, the coding of "speech" rather than "other noise" often depends on how these 'words' are said. See the section on "other noise" for more information.

Neutral Comment. any speech that does not directly refer to the child as a boy or girl. Most speech will fall under this category.

Girl. Any time the mother refers to her child (boy or girl) as a girl. This can includes the word "girl" and any other word directly addressing the child's gender such as "miss," "lady," etc.

Boy. Any time the mother refers to her child (boy or girl) as a boy. This can includes the word "boy" and any other word directly addressing the child's gender such as "little man," "cowboy," "mister," etc.

Positive. Many different things may fall under the category of positive vocalization. Look for celebratory phrases (e.g. yay! Good job!) and clear examples of motherese which feature more variation in frequency and pitch than more flat everyday speech. The difference between motherese considered to be positive vocalizations and neutral/negative vocalizations will be dependent on thresholds designed during reliability. It must be obvious to anyone listening to the tapes that speech defined as positive is positive, as the infant's ability to distinguish between positive and neutral/negative speech is necessary for the speech to have any effect on the responses of the infant. If a period of speech is mixed positive and neutral/ negative speech, code the entire block as positive.

Neutral/Negative. This type of speech does not fall into the category of positive speech, either because it does not pass the thresholds that have been set, or because it does not demonstrate the characteristics of positive speech. When in doubt, code as neutral speech-do not be concerned if much of the vocalization falls under neutral.

Babble to infant. Nonsense syllables either spoken one at a time, or strung together to make word-like compounds, are coded as babbling. (e.g. "do do do ba ba la la la"). Short, quick, slurred repetitions of a single word (e.g. "tickle") should be coded as babble. "Babble" is most commonly found when a mother repeats the babbling of her infant. However, "babble" is also coded in other instances (i.e. spontaneous babbling on the part of the mother that is not in response to infant babbling) and not all vocalizations that a mother makes in response to her infant are coded as "babble." "Other noise" is also common.

If a "babble" is followed, or surrounded by (using the 1.5 second threshold of separation) speech and is less than 1 second in duration, the "babble" should be lumped in with the "speech" code (In other words, only "speech" should be coded). However, "babble" events lasting longer than 1 second should receive its own code. Note that this time threshold for "babble" and "speech" is different from the time threshold used for "other noise" and "speech;" see the section on "other noise" for more information.

Sing. If the mother sings to the infant, whether she is singing real words, humming or babbling with rhythm, it is coded as sing.Coders should be careful not to code rhythmic speech as "sing;" a melody must be present for the vocalization to be sing. However, instances that are borderline should be coded as sing due to its infrequent nature. Whistling is included under Sing. 
Other Noise. Non-syllabic noises such as hissing or shushing or any other vocalization that does not fall under one of the four categories above should fall under the category of other noise. If the other noise is preceded, followed, or surrounded by (using the 1.5 second threshold of separation) speech and is less than 2 seconds in duration, the other noise should be lumped in with the "speech" code. If the noise lasts between 2-3 seconds the noise can be coded either as a separate, "other noise" event or as a part of "speech," depending on the judgment call of the coder. "Other noise" events lasting longer 3 seconds should receive their own code. Food noises, such as "mmm" or "ohmm," should be coded as "other noise." "Yum" or "yummy" should be coded as "speech" unless they are extremely vowelheavy, in which case they should be coded as "other-noise.") However, the noises "hmm?" or "huh?" (see infant 121 for examples) will generally be coded as "speech" unless the noise is extremely brief, grunt-like or clearly not being used as a form of speech.

The difference between "speech" and "other noise" on borderline vocalizations that are not clearly identifiable as either speech or noise (such as "weeeee") depends partly on how vowel/consonant-heavy the vocalization is. For instance, an extremely prolonged "weeeee" with a strong emphasis on the vowel sounds would be coded as "other noise," whereas a shorter "weeeee" (generally under three seconds on length) would be coded as "speech." In general, borderline cases should be skewed towards "speech." A vocalization that is a mixture of speech and laugh will usually be coded as positive. While high pitch is often found in "positive speech," high pitch alone does not make speech positive. Speech that is high-pitched but low in variation/ volume is usually coded as "neutral/negative."

General Rules: If a vocalization happens in the $1^{\text {st }}$ half second of an observation, code the action as beginning at 0:00 for reliability purposes. Maternal vocalization codes should be turned off if there is more than 1.5 seconds between vocalizations. The exception to this rule is if the infant vocalizes in between maternal events. If this happens, an effort should be made to turn the maternal code on and off even if the pause is less than 1.5 seconds. Some discretion should be used (i.e. if the breaks are too short to capture, or if there is overlap between mother and infant vocalization and the mother is clearly not responding to the infant's vocalization).

\section{Coding Manual: Dyadic Play Type (Frame)}

\section{Overview}

This category of codes is designed to capture the motherinfant dyadic play types that frame both maternal and infant play behaviors and interactions. The coding process included two hierarchical steps.

\section{Definitions And Coding Directions}

Object play with infant (guided object play). This code refers to instances in which the mother plays with an object jointly with the infant. For this code the mother takes an active role in demonstrating and scaffolding the infant's use of an object. The mother's actions are directed toward demonstrating an object's properties or supporting an object. The mother or infant may be holding an object. Object use can include games such as tossing a toy back and forth or playing keys on a piano together.

This code should be used whenever a mother and infant both make play-related contact involving an object, except when such contact is clearly accidental. (A mother unintentionally dropping a ball into the infant's lap is an example of "accidental contact".) This code applies when the mother and infant are using the same toy simultaneously, even if they are using different parts of the toy.

Motor-social play (not-guided object play). For this play type (frame) the topic of communication is the participants themselves with no intervening objects. This code captures all instances in which the mother plays using gestures alone (i.e. no toys). Such play can include face-to face play, patty-cake, clapping, or playing peek-a-boo with one's hands, as well as physical motor play such as "bicycle legs" or spreading the infant's arms wide and the closing them together. Presenting hands in an act of encouragement should also be coded under this play type.

Child-directed play (passive or not-guided object play). This play type (frame) records sequences in which the infant plays with objects without the mother's direct assistance but with her ongoing visual attention. She may also at times provide the infant with postural support. However, the mother does not touch the object or act on it in any direct manner. The infant holds the object.

Physical-joint object play mixed. This is coded when elements of face-to face play and guided object play appear at the same time, as when a mother uses a toy to touch 
the infant's face or body while vocalizing in an expressive manner typical of either physical play or social play.

Pushing the infant on a swing or a slide (or similar object) should be coded as "physical-joint object play mixed". If the infant is in a rolling chair and the mother moves the chair back and forth, the mother's action should be coded "physical-joint object play mixed." In this case, the mother and the infant do not focus on the object (e.g., a chair, a ball, a swing etc.) and the mother's intention is to entertain the infant by moving it, or to share the enjoyment with the infant. That is, the mother and the infant socially interact with each other via the mediation of physical play with an object.

Off play-other. Cleaning out the toy without any offering to the infant or transitioning from one play frame to another play frame.

\section{Coding Manual : Maternal Behaviors (Infant motor-directed)}

\section{Table A1-3}

Infant motor directed Maternal behaviors overview (Code:subcode)

\begin{tabular}{l}
\hline Manipulation-direction \\
Offering an object \\
Point \\
Take-shift object \\
Responding \\
Hold object \\
Affectionate touch \\
Gross motor stimulation \\
Assisting locomotion \\
$\quad$ Hold-gross motor \\
$\quad$ Assist-fine motor \\
Shift infant \\
Lifting up \\
Caretaking \\
Rocking/jiggling \\
Observing infant
\end{tabular}

\section{Definitions and Coding Directions}

Manipulation-Direction. Any sort of behavior that uses hands or in an otherwise skillful manner to move, arrange, operate, play or control a toy or an object. This code includes pretend behavior to demonstrate a skill by using a toy. For example, the mother hugs a teddy bear or greets or feeds a doll. Rolling a ball or turning pages of a book can be coded as manipulation. Mothers show or demonstrate how a toy works by holding a toy up or out towards the infant while looking at, gesturing, or verbalizing to the infant.

Offering an object. Offering the infant a toy by handing a toy or an object towards the infant (within arm's reach); rearranging or setting toy down in front of the infant or on its lap; throwing or kicking a ball directly to the infant. The mother may or may not release the toy if the infant tries to accept the toy.

Point. This code captures instances in which the mother points to direct the infant's attention to something. Pointing can involve the use of more than one finger.

Take-shift object. This code should be applied whenever the mother removes a toy from the infant or adjusts or shifts a toy that the infant is playing with. Moving a toy so that it is within the infant's reach should not receive this code; instead it should be coded as "offer." If the mother moves an object that the infant has played with in the recent past (during the same 5-minute segment) but is not currently using, and the infant does not seem to be aware that the mother is moving it, the mother's action is labeled as "holdother." If the infant appears to be aware of the mother's action, it should be labeled as "take-shift." If the infant offers a toy and the mother takes it from the infant, the mother's action is still coded as "take-shift." When a mother moves her infant away from a toy, her action should be coded as "take-shift." This situation is especially common if the toy is stationary (such as a slide). However, shifts of the infant resulting in a toy falling out of the infant's grasp should not be coded as take-shift.

Hold object. This code is designed to capture any other activity the mother does with an object including passively holding it. If a mother is "holding" an object without using her hands (such as placing it in her lap), this "holding" should not be coded. For situations that fall on the borderline between "hold-other" and another code, such as "take-shift," the other code should take precedence. 
Responding. Any behavior that imitates the infant's behavior or accepts or acknowledges the infant's offered or shared toy. More generally, the code refers to any sort of maternal imitative response to an infant-initiated behavior.

Affectional touch. Any touch or behavior that primarily conveys affection, including tickles, nuzzles, kisses, stroking, and hugging. Additionally, stroking of the infant's hair in the absence of a hair decoration, hair clip or brush should be coded as affectional.

Gross motor stimulation. All instances in which the mother moves the infant's limbs so as to mimic infant gross motor behavior such as kicking or flailing the arms. Moving the limbs in the service of some activity that falls under another stimulation code (e.g. caretaking or readjusting position) should not be coded as gross motor stimulation. Restraining the infant's arms or legs or preventing them from moving can, in some instances, be coded as "gross motor stimulation."

Assist locomotion. This code should be applied whenever the mother holds the infant in such a way to facilitate standing, walking, etc. A common instance of this code occurs when a mother supports an infant's hands so they can stand or walk before they are able to do so independently. Assist locomotion also includes times when a mother manipulates a toy with the infant's hand to teach or show how to use or manipulate a toy. This code can be interrupted by other codes (e.g. affectional touch) that reflect a more pronounced physical exchange with the infant.

Holding infant's gross limb. To assist infant's locomotion or movement, the mother holds infant's arms, torso, or legs.

Assisting infant's fine motor/hand. To assist the infant's manipulation or object use, the mother holds the infant's hand or fingers.

Shift infant. This code refers to instances in which the mother repositions the infant. This might include lifting the infant from a sitting to standing position or shifting the infant closer to a toy. Little to no effort should be displayed by the infant. Thus, if the infant's feet clearly leave the ground a shift should be coded. Minor adjustments with little effect on the infant's position (e.g. when a mother is holding an infant and readjusts her grip on the infant or hikes the infant up slightly) should not be coded.
Lift up. This code records instances when the mother lifts the infant into the air as a kind of motor play type. Brief lifts that only serve the purpose of repositioning the infant should not be coded as lift up, but instead should be coded as assist locomotion:shft infant.

Caretaking. This code should be applied when the mother engages physically with the infant to perform a caretaking task, such as wiping the nose, diapering, dressing or adjusting clothing, cleaning, feeding, burping, or protecting (i.e. removing something from mouth, etc.). A sustained caretaking act may involve minor interruptions without requiring that the code be turned on and off. The code should be turned off if the pauses last more than one and $a$ half seconds. For example, during feeding the code should be turned on only when the spoon is in contact with the mouth and therefore should turn off during refilling. Burping, and brief interludes of cleaning during feeding (e.g. wiping the mouth) should also be coded under caretaking. Codes such as affectionate touch that occur simultaneously with caretaking should take precedence over the caretaking code.

Rock/Jiggle. This code refers to instances in which the mother rocks, jiggles, or otherwise rhythmically moves the infant.

Observing infant. This code applies when the mother watches the infant's face or infant's activity.

General rules. Do not code a mother's action (such as bouncing a ball) if it takes place entirely off screen, even if sounds from the action can be heard. However, if an action that is initially shown on screen moves off screen or otherwise out of view can still be heard, it should be coded. Similarly, if an audible action starts off-screen but moves into view, the entire sequence should be coded. Visual evidence that implies that an action took place (such as the moving shadow of an audible bouncing ball) can count as sufficient "on screen visual evidence."

Note. Make a note when a video clip contains a significant amount of play with a swing set or moveable chair. 


\section{Coding Manual: Infant motor behaviors and vocalization}

Table A1-4

Infant motor behaviors and vocalization overview (Code:subcode)

Infant Gross Motor Behavior
Lying:
Roll over
Kick/Leg move
Still
Sitting:
Independent
Assisted mom
Assisted other
Rock/Lean/Bounce
Crawling
Standing:
Independent
Assisted mom
Assisted other
Rock/Lean/Bounce
Walking:
Independent
Assisted mom
Assisted other
Reaching:
To grasp/touch
To offer or show
Other reaching
Pointing

Infant Motor (other)

Bat-bang-clap-flail

Reach

Kick

Other gross motor

Infant Vocalization:

Babble

Cry/Fuss

Word

No Vocalization

\section{Definitions And Coding Directions}

Infant motor behaviors are designed to capture the postural and locomotive status and gross motor limb movement of the infant. Big five locomotion or activities (Lying, Sit, Crawl, Stand, Walk) reflect whole body activity or locomotion, while the remaining three activities (Reaching, Bat/Banging, Pointing) reflect upper body, that is, shoulder, arm, elbow, and hand movements. Pauses in active states which last less than 1.5 seconds will not be coded as interruptions of the active state. (e.g. An infant who is crawling for 4 seconds, then pauses in crawling position for 1 second, then continues to crawl for another 5 seconds should be coded as having crawled for the entire 10 seconds.) In contrast, a short burst of activity should be coded regardless of duration. When an infant is transitioning from one locomotion/position (e.g. sitting to walking) to another, coding switches over when the new activity begins (e.g. first step), not when the old activity ends. Long transitions that contain codeable intermediary motions or positions should be parsed and coded as separate behaviors.

Lying. This code captures all instances in which the infant is lying on the front, on the back or side, or is rolling over. Although infants sitting in rockers, car seats, etc may not be fully upright, these instances will still be coded as sitting, rather than lying on the back, unless the infant is extended nearly horizontally.

Still. When the infant does not move and his/her position is static, lying is coded as still.

Roll over. When the infant rolls over- whether rolling from front to back, back to front, or rolling for an extended time on his/her side. The threshold for this code is 90 degrees.

Kick-Leg movement. When the infant is lying, the infant displays any medium to large gross motor movement of the legs. As with "flail", it includes clear leg limb extensions caused by imprecise muscle flexing or twitching. The movements should be either prominent or repetitive.

Sitting. The infant will be coded as sitting whenever he or she is in an upright position and supported on the bottom or legs. Sitting on a lap, in a swing, car seat, or any other apparatus is included in this code. This code also includes instances in which the infant is sitting on its knees in an upright position and not supported by the hands. (Instances in which the infant is strapped to the parent will be coded as Held and not Sit.)

Independent. Instances in which the infant is seated anywhere without additional support. Instances in which the infant is sitting on his/her hip/bottom and weight is supported by one or both arms and the hip/bottom should be included in this modifier. This modifier does not include sitting on the mother's lap or in a seat designed to assist the 
position (such as a baby swing or a walker. These should be coded as sit: assisted other).

Assisted mother. Infant sits with support (typically on trunk) from mother.

Assisted other. Infant sits, typically in a seat with back support. Instances where an infant is in a walker and is stationary with the feet not supporting his/her weight fall under this modifier. Also, use this modifier for all instances in which the infant is seated in a high chair or a swing with a back. (If the infant is sitting at the top of a slide, code sit: independent)

Rock/Lean/Bounce. When the infant is sitting independently or sitting on another chair or on a swing, and the infant shows prominent movement of posture or position. Examples include rocking back and forth or bouncing up and down in a crawl position. The key to this code is repetitive movements. If the infant is in an assisted seat or walker and initiates rocking, leaning, or bouncing movements, use this code. However, instances in which the mother bounces the infant fall under the maternal stimulation code rock/jiggle. This code does not include instances of limb movement while the trunk is stationary.

Crawling. This code captures all instances in which the infant moves directionally by some means other than walking. This includes the typical hands and knees version of crawling but is not limited to such expressions of crawling (e.g. includes locomoting on hands and feet). Scooting or any other means by which the infant manages to pull him/ herself around (aside from walking) qualify for this code. It also includes the stationary crawling position.

Standing: The infant will be coded as standing when he or she stands upright on the feet, without walking. This may occur with or without the support of an object or adult.

Independent. The infant stands without holding onto his or her mother or any object (e.g., chair, couch, table edge). Instances in which the infant is merely resting a hand on an object (a table, for example), and not using it for assistance in the stand position fall under this code

Assisted mother. The infant stands and the mother assists by holding the infants hands or trunk.

Assisted other. The infant stands while holding onto something, such as a table, chair, etc., for support. This modifier also captures instances in which an infant is in a seat or walker and both feet are on the ground, and are supporting the infant's weight (if no weight is supported, this should be coded as sit: assisted other). If the infant is clearly leaning on an object such as a table, and using its trunk, but not hands for support, this falls under this modifier. However, if the infant merely has a hand on a table, but is not using the table to assist in standing, this should be recorded as stand: independent.

Rock/Lean/Bounce. When the infant is standing with assistance or independently and shows prominent movement of posture or position. Examples include rocking back and forth or bouncing up and down in a crawl position. The key to this code is repetitive movements. If the infant is in an assisted seat or walker and initiates rocking, leaning, or bouncing movements, use this code. However, instances in which the mother bounces the infant, fall under the maternal stimulation code rock/jiggle. Instances of limb movement while the trunk is stationary do not fall under this code.

Walking. This code captures all instances in which the infant moves directionally with alternating steps (at least one alternation). The infant may be aided by an object or adult but must, him or herself, be responsible for leg movement and at least partial support of weight.

Independent. The infant walks without the assistance of the mother, an object for support (couch, table), or a walker

Assisted mother. Mother assists in the locomotion, by holding the infant's hands, supporting his/her body, etc

Assisted other. The infant walks with the assistance of another object, such as the edge of a couch, a walker, etc (note: with a walker, must alternate steps to be coded as walk: assisted other)

Bat, Clap, Bang, Flailing. In contrast to reaching, batting consists of efforts to hit or bang an object, or it consists of repetitive medium-to-large arm movement that is not directed at a specific object (e.g., flailing). Batting will generally refer to instances in which the infant repeatedly hits an object with his/her hand. If the infant is holding an object and banging it against something else, it should receive this code. To receive this code, the infant (or toy) must clearly or repetitively contact something. Brief incidental contact is not sufficient to warrant this code, but, single brief contacts involving the palms/hands/fists or an object can be coded provided they are clear and distinct. If contact is sustained with an object, it should be coded as a reach, rather than a bat. A bat should involve both hitting and retracting (although it can include retracting from the object to hit it again in the same place or one very close to it).

Pointing. Points for which both arm and finger are extended in the direction of the object of interest. 
Reaching. This code captures all instances in which the infant extends an arm to grasp or get an object, or to offer or show an object. The beginning of a reach is coded when the first movement in the service of the reach is evident, even if this movement is postural or in the opposite direction of the reach. The end of the reach is coded when the infant makes contact with the object or gives up reaching for the object. (Note that successful grasping isn't necessary to receive a reach code). A reach can be coded without large-scale arm movement, if the arms are extended and clearly straining toward an object or a person. Reaching can include both hitting and retracting from the object to hit it again in the same place or one very close to it.

To grasp or to touch the object. This code applies to the infant's arm extension to grasp or to touch toy or an object.

To offer or to show the objects. This code applies to the infant's arm extension to offer or show a toy or an object to the mother.

Other. When the infant's arm reaching is not clearly toward an object or a person, the behavior is coded as other.

\section{Specific Rules for coding "Reaching".}

- If smaller reaching occurs for an amount of time slightly longer than the 1.5 second grace period, even if this reaching would normally not be coded, leave the code on if the infant begins reaching again afterwards. This does not apply if the intervening reaching is primarily fine motor rather than gross motor based

- In a sequence of reaches, do not bother coding the pull backs as "other." Just leave the reach code on. However, if the pull back is distinguishable from the reach because of the duration of intervening time or the length of the pullback (e.g. if the pullback is just as long as the reach, it will probably distinguish itself as its own code under other).

- While the object being reached for does not necessarily need to be touched, it must be clear that the infant has the hand position to either touch or grasp the object. Incidental contact, therefore, by an infant hand that is not in the appropriate conformation to grasp or touch does not cause a movement to qualify as a reach.

- Reaches must be clearly intentional by some sort of visible orientation towards the object being reached for. This can come in the form of bodily or visual orientation in the direction of the object. When in doubt, do not code as reach.

- If the infant is already holding an object and extends the same arm holding that object to either displace the object onto another spot, the movement should be coded under other. However, if the infant is already holding an object and extends the same arm holding that object to displace the object in a smaller spot (which means that the movement requires more precision, such as dropping a toy piece into a corresponding small hole) the movement should be coded under reach. Other examples of reaching include movements of the arm to put objects into the mouth, to stack small objects on top of each other, or when placing back pieces into a puzzle-like toy.

- A small arm or small leg movement such as muscle twitching or flexing at one time, need not be coded. But 1) when these movements last over 1.5 seconds even though the flexing or twitching is small, 2) when these movement occur independently over 1.5 seconds between multiple gross motor movements, or 3) when these movements occur very clearly, they should be coded. When these movementsare repetitive, they should be coded even though the duration of the specific behavior does not last over 1.5 seconds.

\section{Notes for reliability.}

- A reach has to be obvious and the movement comes from the shoulder. If the reach looks as if it originates from the elbow, it is not a reach.

- When two events occur simultaneously the longer event is coded, i.e., if a reach occurs while the baby is flailing and has been flailing for a longer period, then the reach is not coded and the flailing code continues.)

- Try to be exact with your start and stop coding because it makes a difference in terms of reliability.

- All of the events you code must be obvious, clear, and must follow aforementioned standards. 
- There must be clear intent involved in any action that is coded. ( if something appears to be caused by gravity, it should not be coded)-follow the gaze.

- A crawl is defined by hand and knee contact. If an infant is not moving, but both hands and knees are on the ground, this is a stationary crawl. If the infant is on both hands and both feet, this is NOT a crawl. No big five action should be coded as a crawl.

\section{Infant vocalization.}

Babble. This code refers to all non-distressed, syllabic vocalizations where clear words can not be easily distinguished by the coder. This includes squeals, squeaks, etc. If there is an aspiration (grunt) that directly precedes and turns into a babble, the whole vocalization can be coded as babble. Vegetative sounds (e.g., sneezes, coughs, and hiccups), effort sounds (e.g., grunts), and negative vocalizations (e.g., fusses and cries) should be excluded from babble.

Cry-Fuss. This code captures all instances in which the infant's vocalizations indicate distress. Distressed facial expression (e.g. furrowed brow) during vocalization is sufficient to warrant the Cry-Fuss code. This can include a closed mouth whine.

Word. This code refers to infant's word used. Without mother's repetition or rephrase, the infant's word must be understandable to the coder. An unfinished syllable cannot be included as a word. However, if the child uses a different pronunciation of a word (for example, "raffe" for "giraffe" or "sketti" for "spaghetti"), code the word.

\section{References}

Efron, B. (2005). Bayesians, frequentists, and scientists. Journal of the American Statistical Association, 100, 1-5. doi:10.1198/016214505000000033

Efron, B. (2013). A 250-year argument: Belief, behavior, and the bootstrap. Bulletin of the American Mathematical Society, 50, 129-146. doi:doi=10.1.1.306.4592\&rep=rep1\&type=pdf

Teng, D. W., Wallot, S., \& Kelty-Stephen, D. G. (2016). Single-word recognition need not depend on single-word features: Narrative coherence counteracts effects of single-word features that lexical decision emphasizes. Journal of Psycholinguistic Research, 45, 1451-1472.
Wicherts, J. M., Veldkamp, C. L. S., Augusteijn, H. E. M., Bakker, M., van Aert, R. C. M., \& van Assen, M. A. L. M. (2016). Degrees of freedom in planning, running, analyzing, and reporting psychological studies: A checklist to avoid p-hacking. Frontiers in Psychology, 7. doi:10.3389/ fpsyg.2016.01832

\section{Appendix 2}

\section{Contents}

A. 3-D state-space for maternal behaviors: assist locomotion, vocalization and affectionate touch for month groups 7-8, 9-10 and 11-12. See main text for discussion of graphing methods.

Figure A2-1: A.

A scatterplot revealing the three-dimensional state space at 7-8 months for duration of the maternal behaviors assisted locomotion ( $x$-axis), vocalization ( $y$ axis) and affectionate touch (z-axis) divided by mothers of sons (marked male) and mothers of daughters (marked female). The blue circles and red triangles represent individual mother-infant dyads. In B: the mesh indicated as male is presented as single color with lines. The mesh indicated as female is presented as a gradient with the gradient scale indicative of $z$-axis values.

\section{Figure A2- 2: A.}

A scatterplot revealing the three-dimensional state space at 9-10 months for duration of the maternal behaviors assisted locomotion ( $x$-axis), vocalization ( $y$ axis) and affectionate touch (z-axis) divided by mothers of sons (marked male) and mothers of daughters (marked female). The blue circles and red triangles represent individual mother-infant dyads. In B: the mesh indicated as male is presented as single color with lines. The mesh indicated as female is presented as a gradient with the gradient scale indicative of z-axis values.

\section{B. Figure A2-3: A.}

A scatterplot revealing the three-dimensional state space at 11=12 months for duration of the maternal behaviors assisted locomotion ( $x$-axis), vocalization ( $y$ axis) and affectionate touch (z-axis) divided by mothers of sons (marked male) and mothers of daughters (marked female). The blue circles and red 
triangles represent individual mother-infant dyads. In B: the mesh indicated as male is presented as single color with lines. The mesh indicated as female is presented as a gradient with the gradient scale indicative of $z$-axis values.

Final cluster centers and average duration for maternal behaviors assist locomotion, vocalization and affectionate touch. See main text for discussion of k-means cluster analysis.

\section{Figure A2-4:}

Final cluster centers: 5-6 months duration. The table lists average number of seconds per five-minute coding sequence for each of three maternal behaviors. The histogram indicates the number of dyads with male infants and the number of dyads with female infants in each of the clusters listed in the cluster table.

\section{Figure A2-5:}

Final cluster centers: 7-8 months duration. The table lists average number of seconds per five-minute coding sequence for each of three maternal behaviors. The histogram indicates the number of dyads with male infants and the number of dyads with female infants in each of the clusters listed in the cluster table.

\section{Figure A2-6:}

Final cluster centers: 9-10 months duration. The table lists average number of seconds per five-minute coding sequence for each of three maternal behaviors. The histogram indicates the number of dyads with male infants and the number of dyads with female infants in each of the clusters listed in the cluster table.

\section{Figure A2-7:}

Final cluster centers: $11-12$ months duration. The table lists average number of seconds per five-minute coding sequence for each of three maternal behaviors. The histogram indicates the number of dyads with male infants and the number of dyads with female infants in each of the clusters listed in the cluster table 


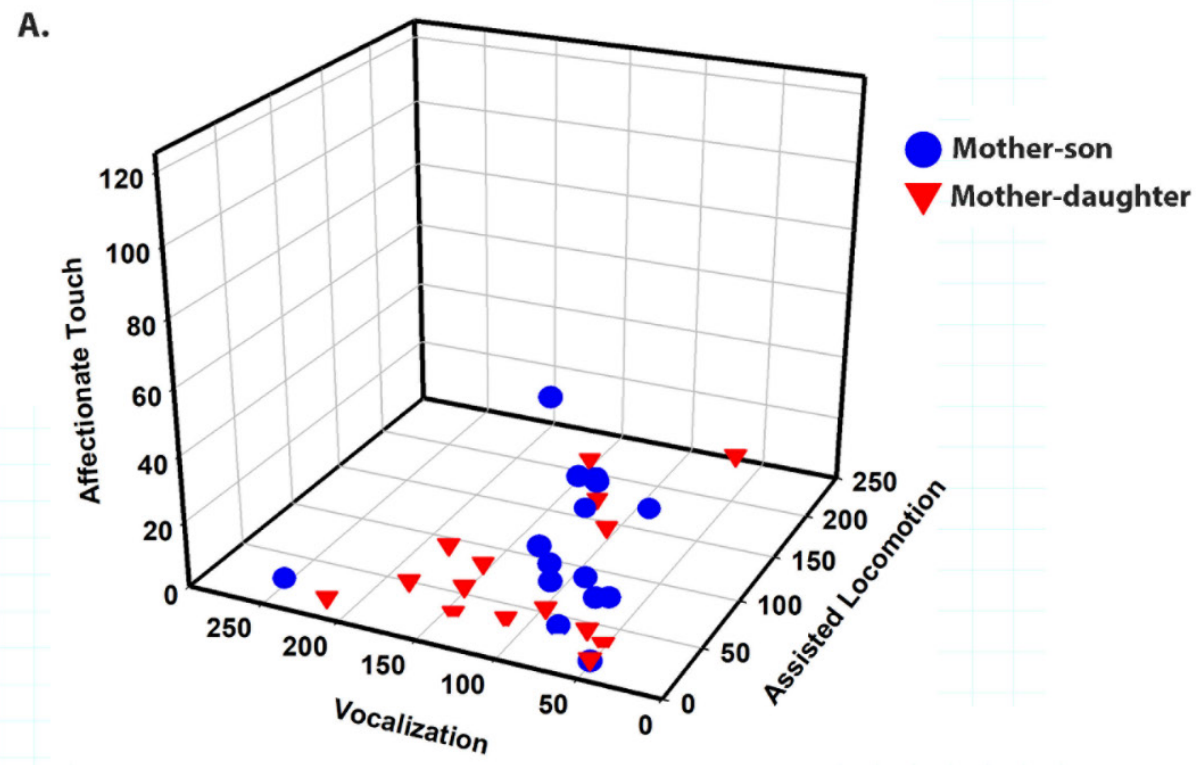

B.

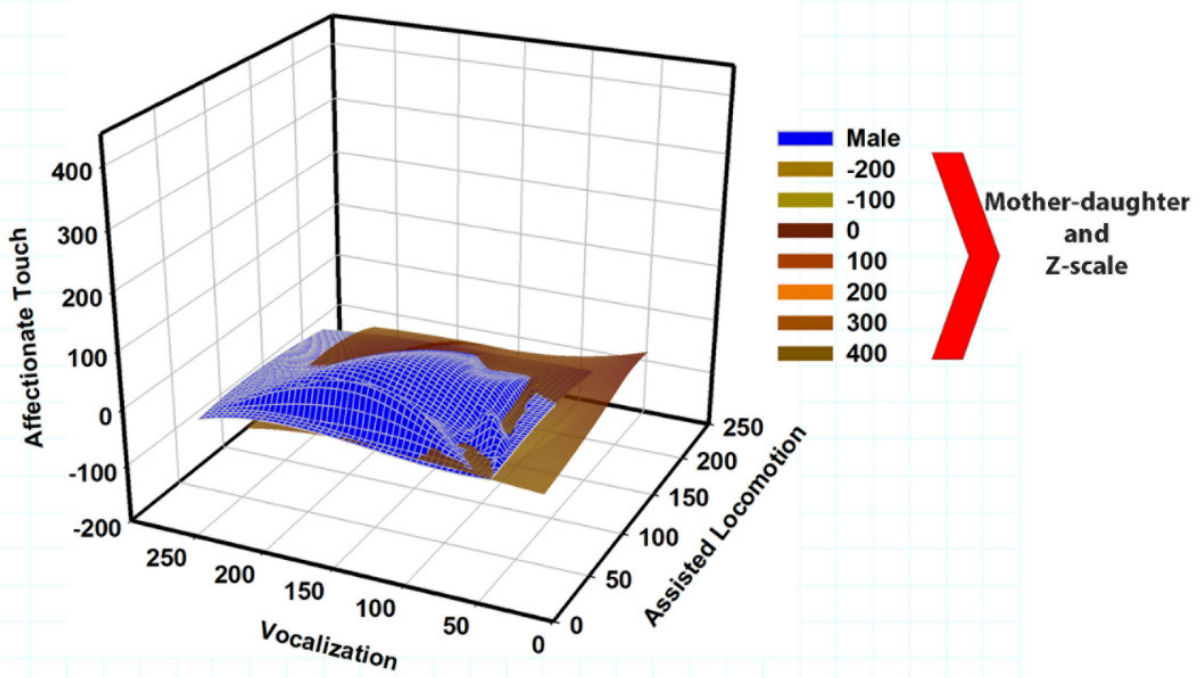

7-8 Months

Figure A2-1. A: scatterplot revealing the three-dimensional state space at 7-8 months for duration of the maternal behaviors assisted locomotion ( $x$-axis), vocalization ( $y$-axis) and affectionate touch ( $z$-axis) divided by mothers of sons (marked male) and mothers of daughters (marked female). The blue circles and red triangles represent individual motherinfant dyads. B: the mesh indicated as male is presented as single color with lines. The mesh indicated as female is presented as a gradient with the gradient scale indicative of $z$-axis values. 
A.

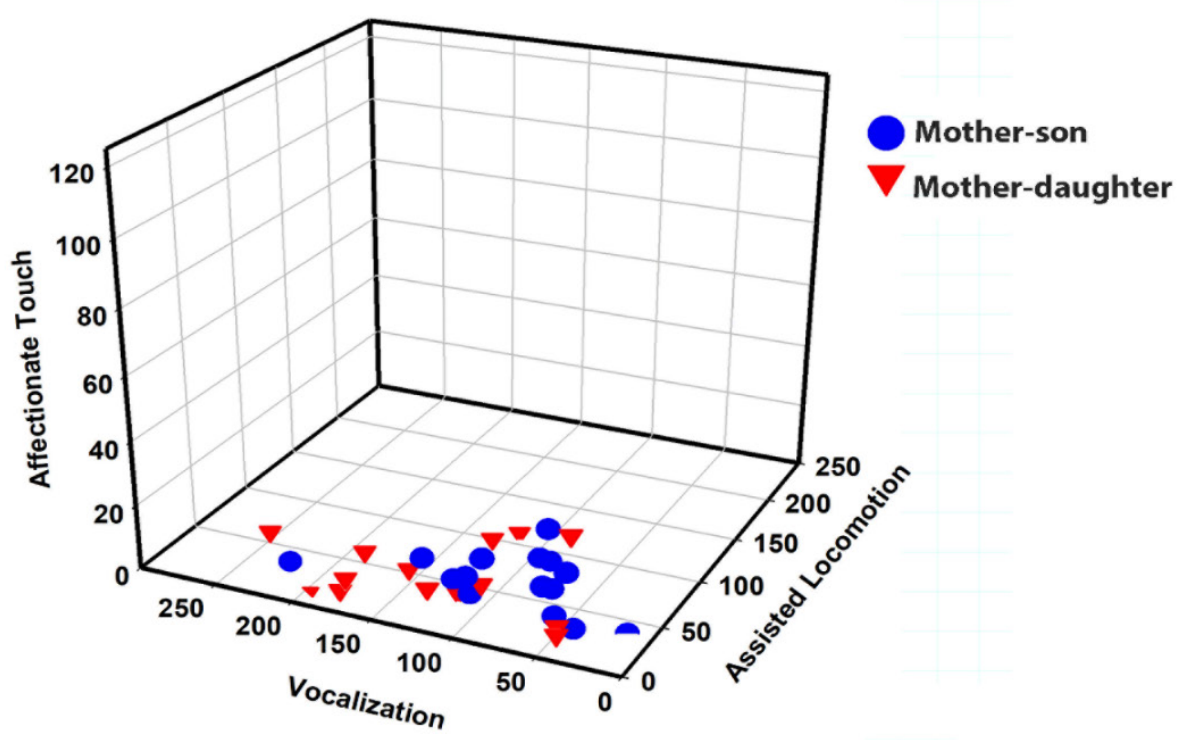

B.

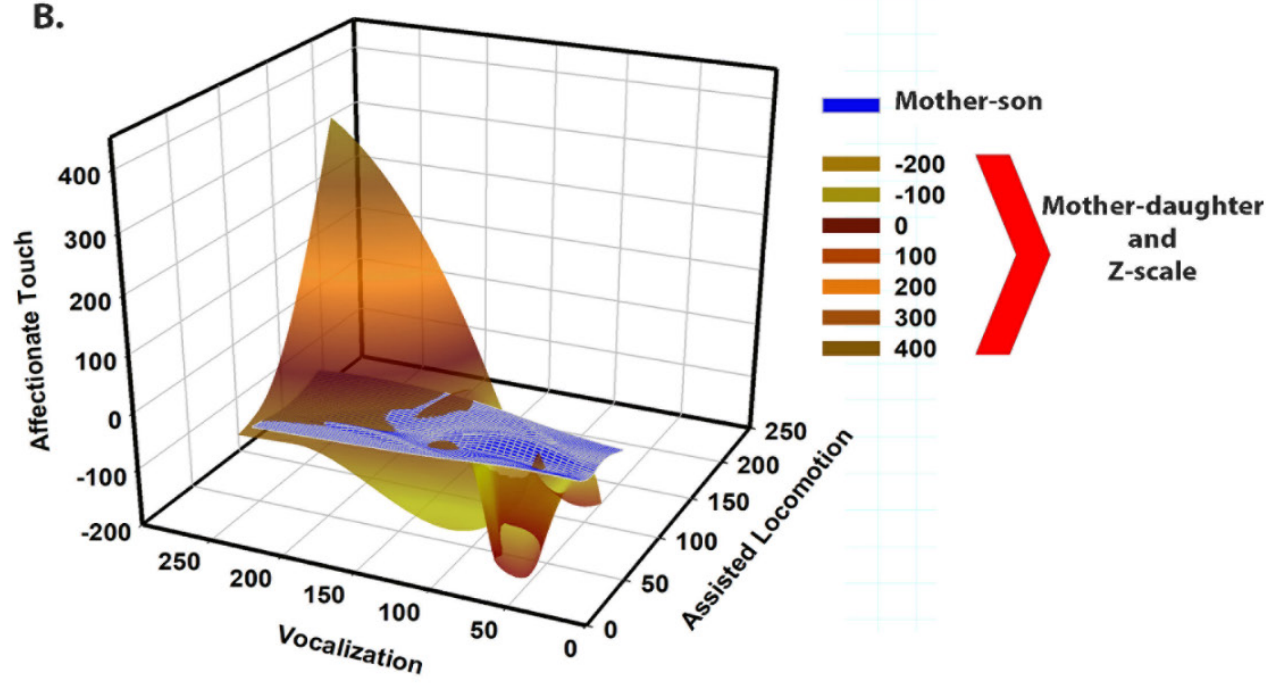

9-10 Months

Figure A2-2: A. A scatterplot revealing the three-dimensional state space at 9-10 months for duration of the maternal behaviors assisted locomotion ( $x$-axis), vocalization ( $y$-axis) and affectionate touch ( $z$-axis) divided by mothers of sons (marked male) and mothers of daughters (marked female). The blue circles and red triangles represent individual motherinfant dyads. B: the mesh indicated as male is presented as single color with lines. The mesh indicated as female is presented as a gradient with the gradient scale indicative of $z$-axis values. 

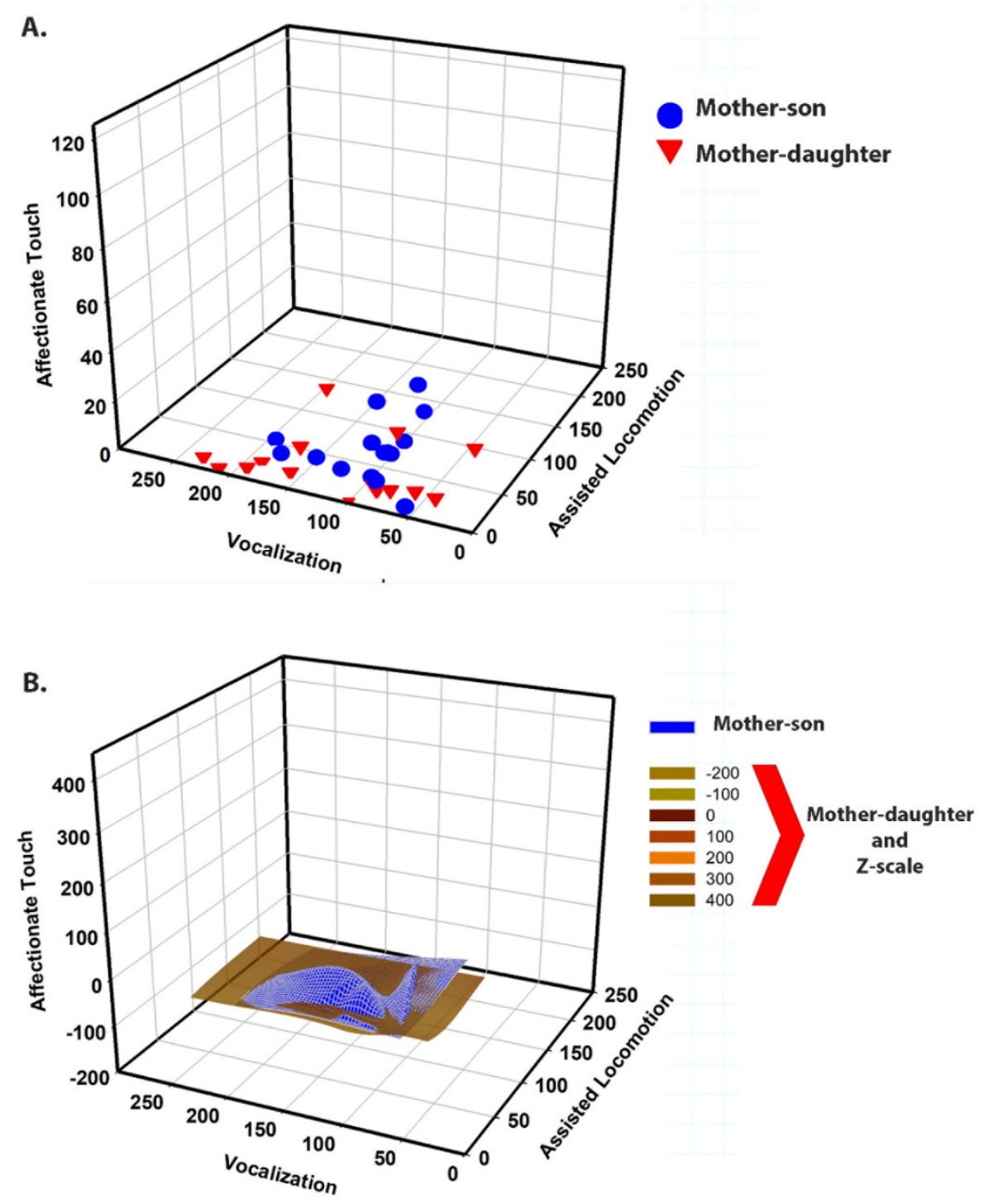

11-12 Months

Figure A2-3: A. A scatterplot revealing the three-dimensional state space at 11-12 months for duration of the maternal behaviors assisted locomotion ( $x$-axis), vocalization ( $y$-axis) and affectionate touch ( $z$-axis) divided by mothers of sons (marked male) and mothers of daughters (marked female). The blue circles and red triangles represent individual motherinfant dyads. B: the mesh indicated as male is presented as single color with lines. The mesh indicated as female is presented as a gradient with the gradient scale indicative of $z$-axis values. 


\section{Final Cluster Centers 5-6 Months Duration} (Seconds)

\begin{tabular}{lr|r|r} 
& \multicolumn{3}{c}{ Cluster } \\
\hline Maternal Vocalization & 114.60 & \multicolumn{1}{c}{2} & \multicolumn{1}{c}{3} \\
\hline Assisted Locomotion & 102.36 & 48.69 & 169.78 \\
\hline Affectionate Touch & 12.37 & 16.30 & 59.41 \\
\hline
\end{tabular}

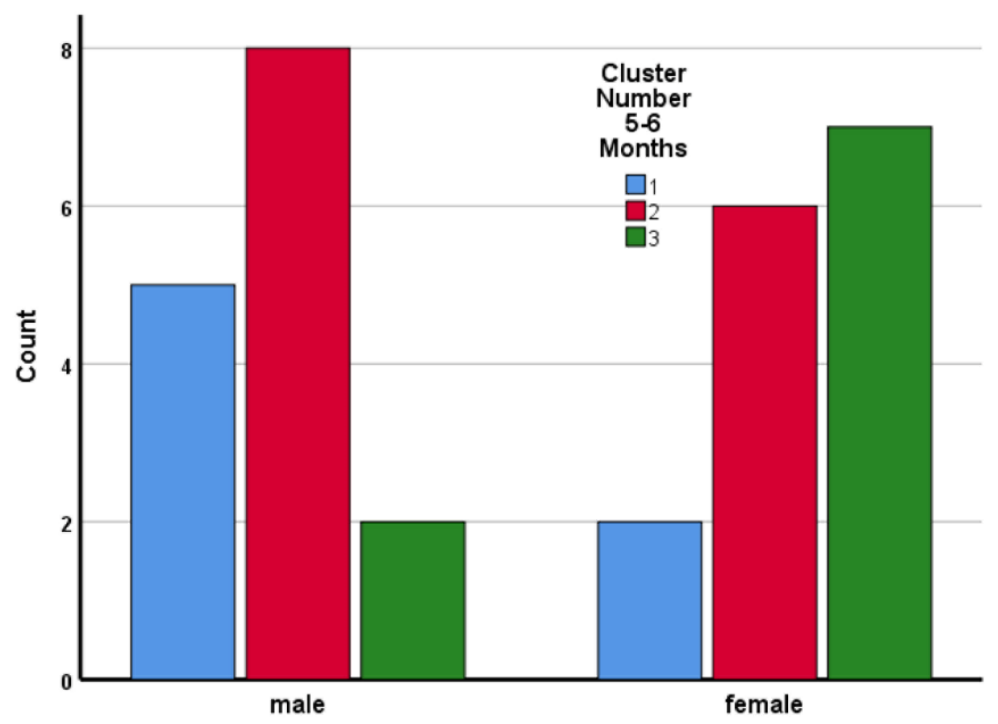

Figure 4: 5-6 Months

Figure A2-4: Final cluster centers: 5-6 months duration. The table lists average number of seconds per five-minute coding sequence for each of three maternal behaviors. The histogram indicates the number of dyads with male infants and the number of dyads with female infants in each of the clusters listed in the cluster table. 
Final Cluster Centers 7-8 Months Duration (Seconds)

\begin{tabular}{lr|r|r} 
& \multicolumn{3}{c}{ Cluster } \\
\hline Maternal Vocalization & 1 & \multicolumn{1}{c}{2} & \multicolumn{1}{c}{3} \\
\hline Assisted Locomotion & 74.63 & 170.10 & 89.37 \\
\hline Affectionate Touch & 37.97 & 26.88 & 128.12 \\
\hline
\end{tabular}

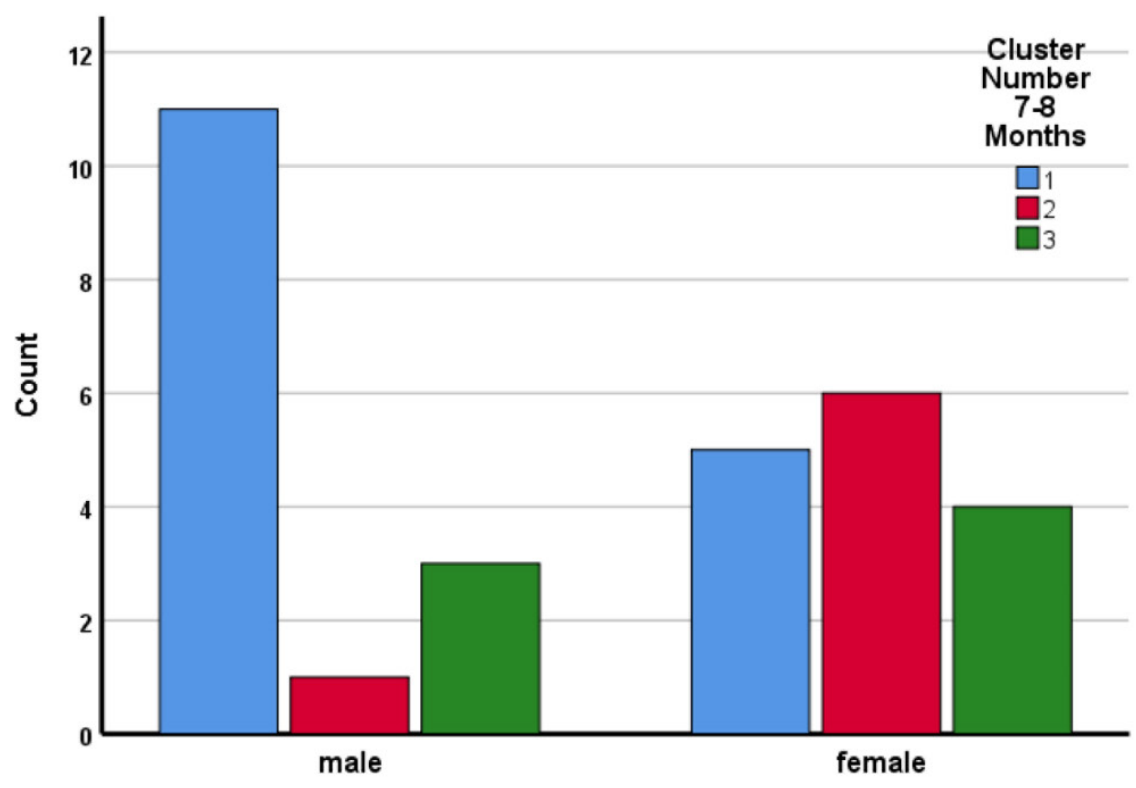

7-8 Months

Figure A2-5: Final cluster centers: 7-8 months duration. The table lists average number of seconds per five-minute coding sequence for each of three maternal behaviors. The histogram indicates the number of dyads with male infants and the number of dyads with female infants in each of the clusters listed in the cluster table. 
Final Cluster Centers 9-10 Months Duration

(Seconds)

\begin{tabular}{lr|r|l} 
& \multicolumn{3}{c}{ Cluster } \\
& \multicolumn{1}{c}{1} & \multicolumn{1}{c}{2} & \multicolumn{1}{c}{3} \\
\hline Maternal Vocalization & 111.24 & 191.76 & 49.50 \\
\hline Assisted Locomotion & 44.17 & 17.67 & 23.95 \\
\hline Affectionate Touch & 7.73 & 8.47 & 12.26 \\
\hline
\end{tabular}

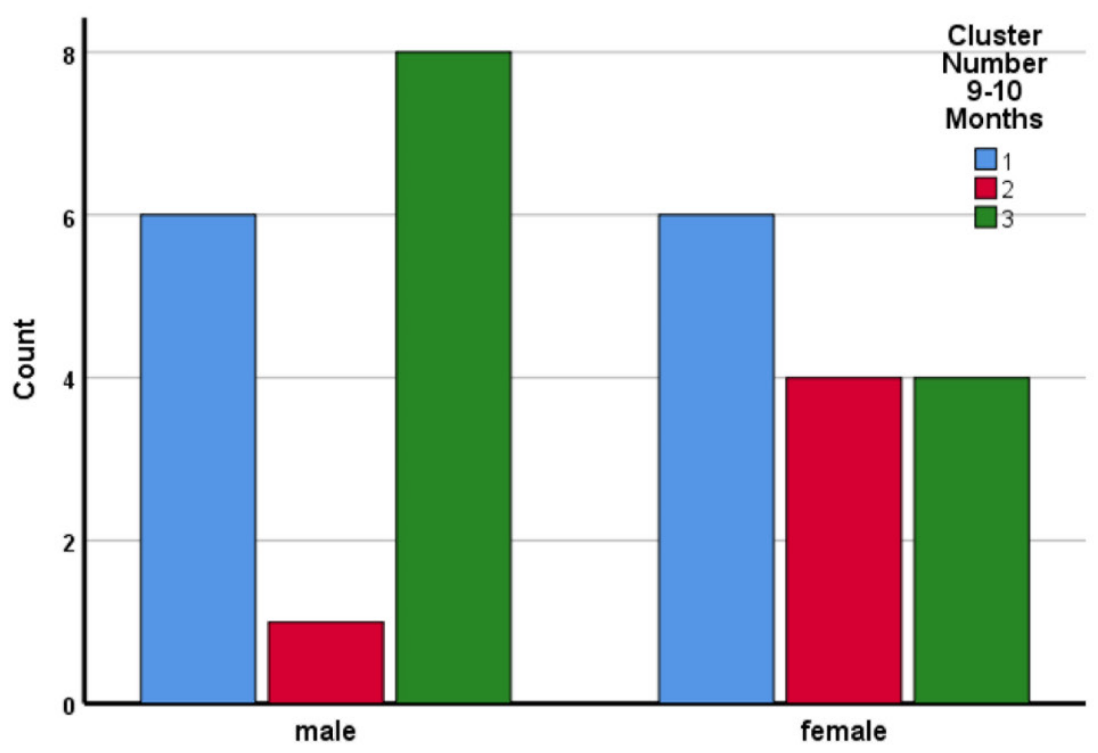

9-10 Months

Figure A2-6: Final cluster centers: 9-10 months duration. The table lists average number of seconds per five-minute coding sequence for each of three maternal behaviors. The histogram indicates the number of dyads with male infants and the number of dyads with female infants in each of the clusters listed in the cluster table. 


\section{Final Cluster Centers 11-12 Months Duration (Seconds)}

\begin{tabular}{lr|r|r} 
& \multicolumn{3}{c}{ Cluster } \\
& \multicolumn{1}{c}{1} & \multicolumn{1}{c}{2} & \multicolumn{1}{c}{3} \\
\hline Maternal Vocalization & 75.96 & 179.03 & 105.34 \\
\hline Assisted Locomotion & 23.95 & 16.42 & 73.05 \\
\hline Affectionate Touch & 6.79 & 4.59 & 14.35 \\
\hline
\end{tabular}

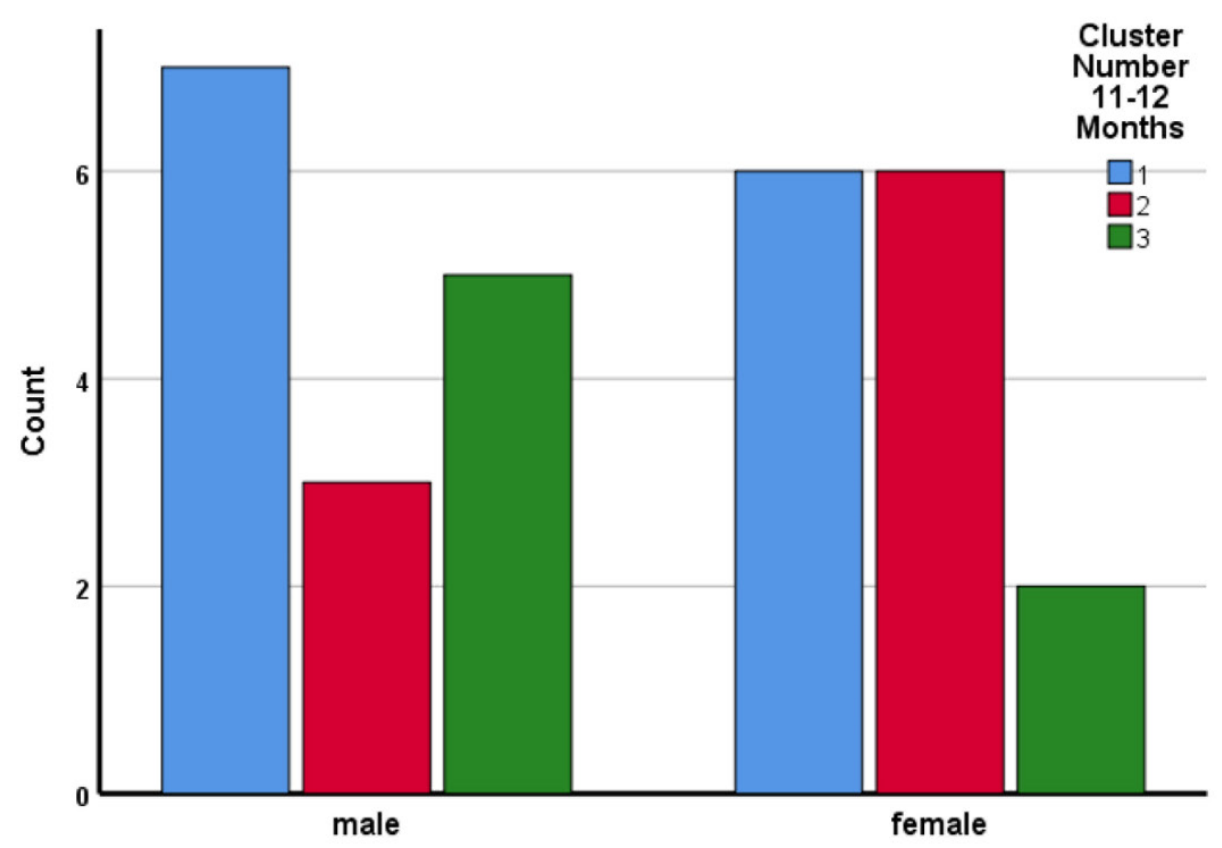

Figure 7: 11-12 Months

Figure A2-7: Final cluster centers: 11-12 months duration. The table lists average number of seconds per five-minute coding sequence for each of three maternal behaviors. The histogram indicates the number of dyads with male infants and the number of dyads with female infants in each of the clusters listed in the cluster table. 